Florida International University

FIU Digital Commons

FIU Electronic Theses and Dissertations

University Graduate School

6-4-2019

\title{
Evolutionary Expansions and Neofunctionalization of Ionotropic Glutamate Receptors in Cnidaria
}

Ellen G. Dow

Florida International University, edow002@fiu.edu

Follow this and additional works at: https://digitalcommons.fiu.edu/etd

Part of the Bioinformatics Commons, Cell Biology Commons, Cellular and Molecular Physiology Commons, Evolution Commons, Integrative Biology Commons, Marine Biology Commons, Molecular and Cellular Neuroscience Commons, and the Molecular Biology Commons

\section{Recommended Citation}

Dow, Ellen G., "Evolutionary Expansions and Neofunctionalization of lonotropic Glutamate Receptors in Cnidaria" (2019). FIU Electronic Theses and Dissertations. 4270.

https://digitalcommons.fiu.edu/etd/4270

This work is brought to you for free and open access by the University Graduate School at FIU Digital Commons. It has been accepted for inclusion in FIU Electronic Theses and Dissertations by an authorized administrator of FIU Digital Commons. For more information, please contact dcc@fiu.edu. 


\section{FLORIDA INTERNATIONAL UNIVERSITY}

Miami, Florida

\section{EVOLUTIONARY EXPANSIONS AND NEOFUNCTIONALIZATION OF IONOTROPIC GLUTAMATE RECEPTORS IN CNIDARIA}

A dissertation submitted in partial fulfillment of

the requirements for the degree of

DOCTOR OF PHILOSOPHY

in

BIOLOGY

by

Ellen Grace Dow

2019 
To: Dean Michael R. Heithaus

College of Arts, Sciences and Education

This dissertation, written by Ellen Grace Dow, and entitled Evolutionary Expansions and Neofunctionalization of Ionotropic Glutamate Receptors in Cnidaria having been approved in respect to style and intellectual content, is referred to you for judgment.

We have read this dissertation and recommend that it be approved.

Matthew DeGennaro

Laura Serbus

Kalai Mathee

Virginia Weis

Mauricio Rodriguez-Lanetty, Major Professor

Date of Defense: June 4, 2019

The dissertation of Ellen Grace Dow is approved.

Dean Michael R. Heithaus

College of Arts, Sciences and Education

Andrés G. Gil

Vice President for Research and Economic Development and Dean of the University Graduate School

Florida International University, 2019 
(C) Copyright 2019 by Ellen Grace Dow

All rights reserved. 


\section{DEDICATION}

I dedicate this to my nurturers.

To my mother, Janet Kvanvig Sappington, who reminds me why I am passionate about each thing I do and treats me as her equal.

To my father, Roger Dow, the mighty oak who has shown me my roots and given me the support and spirit for this acorn to sprout so far away from home.

To Cortnie Haun, who became completely integrated within family during this journey and has always given me patience.

To my grandparents Jerome and Jacqueline Kvanvig, who taught me that it was a gift to be an artist and a scientist, along with my first compound microscope and visits to the Oregon Museum of Science and Industry.

To my grandmother Isabel McCluskey Dow, who taught me to love and care for all creatures and to my grandfather, Ronald Charles Dow, as his first grandchild to graduate college and then immediately jump into another degree, I hope to have brought you pride and joy. I miss you deeply.

To my lifelong friend, my chosen sister, Madeline Robinson, who has been there for the past 21 years and has given me a place to sleep in San Francisco whenever I need it. Thank you for the fun and the traditions.

To my treasured friend and college roommate, Lindsay McAfee, who amazed me with all of her accomplishments throughout college and after with such humility. Thank you for teaching me how to be grounded and that friendship is worth more than dirty dishes.

To my fairy godmother, Tami Hale Bogat, who taught me to follow my heart. 
To the entirety of my Miami family, I could not have completed this without your love and support.

To my Miami parents, Mark and Jen Durocher, who quickly took me under their wing.

To my dear friend Aubree Hibbe, who has brought beauty and nature into my life.

To my running family here in Miami, Antoine and Sharon Wilson-Dodard, for yoga and always being up for a beer. To my running partners Ron Saint-Albin, Cecile Warshaw, Abbe Kerr, Dawn Aran-Othon, Jose Mercado and my running family Max Desse, Ceci O’Reilly, Hilary Jemeson, Ari Dorfsman, Patricia Price, Judy Flook, Karine Stengel, Jill Zemel, Robert Riascos, Gae Shapiro, Gladys Noriega Zavala for each helping me in some way here in Miami. 


\section{ACKNOWLEDGMENTS}

For this dissertation to exist I have many people to thank for their guidance and help through the past five years. Thank you to my major advisor, Mauricio Rodriguez-Lanetty for welcoming me into his lab, providing funding for my research, and encouraging my independence and drive to grow as a scientist. Thank you Mauricio for also being so kind during my $\mathrm{PhD}$, moving from Oregon, breaking my elbow and losing a grandparent in the span of a month was very hard and I thank you for all the patience and comfort in that time, as well as the post-surgery jello.

I have so much gratitude for my committee members who ensured that I had a broad range of knowledge coming into this work and supported the challenging tasks I had in front of me. Their time committed to reading my proposal and dissertation has contributed to improving my writing and analyses. Dr. Matt DeGennaro provided so much insight into iGluRs, gave me a crash course as I dove into this area of research and pushed my thinking of the scope of how iGluRs function in context of cnidarians, along with his invaluable support for my fourth chapter, which would not exist otherwise, and all the in situs I completed this past year. Dr. Laura Serbus broadened my views of symbioses, helping me think beyond corals and inspiring me to use art in my communication of knowledge, and so much support in this last year while I felt so overwhelmed. Dr. Kalai Mathee ensured I looked at my research and methods with a critical eye. Dr. Virginia Weis provided so much experience and knowledge in the field of Aiptasia molecular research and discernment to my project. I am so glad to have her as a mentor since 2011, when she took me in to her lab and took a chance on me, allowing 
me to right my first proposal for undergraduate research. And thank you to the Weis lab, for welcoming me back during conferences. To members past and present, my first mentor Dr. Angela Poole, my conference guru Dr. Sheila Kitchen, Trevor Tivey, Jack Koch, Shumpei Maruyama, and Jason Presnell for friendship and time to consult on my research.

Thank you to my lab mates from the IMaGeS lab. During this process, everyone has given me their time and attention, especially while practicing my talks. Dr. Anthony Bellantuono, thank you so much for your expertise in cloning and always being in the lab to help me trouble shoot, you are an incredible scientist and are so remarkably knowledgeable about nearly anything. Patty Waikel for her support and always being up for a coffee break. Thank you to my undergraduates for their help. Thank you Mercy Kim for assisting me with getting cloning underway and so many PCRs on colonies to check for inserts, Melissa Lagunas (ML) for learning RNA extractions and cDNA synthesis to help process the 60 samples for the diurnal experiment and to Beatriz Maderal (BM) for learning so quickly how to process samples and load 90 well plates and becoming an amazingly independent assistant. In the background on AHC-1 second floor, thank you to Dr. Erasmo Perrera for helping me get my hands on any reagents I needed at last minute. Dr. Marcela Nouveza for company while I shared lab space and use of the microscope, and supporting my anxiety baking habits. And to the Biology office staff, who made sure I received my travel funding and had all my paperwork in line.

Thank you Dr. Jessica Siltberg-Liberles (JSL), for helping me through getting my phylogenetics analysis right. Thank you for spending the time to walk me through the 
first steps and always providing prompt feedback. And thank you for the editing help, to truly synthesize my first chapter.

Thank you Dr. Ann Tarrant (AMT) for confirming and helping me think about my diurnal research design. Thank you for all of the feedback, especially the advice to explore the symbiotic diurnal research data, which strengthened my analysis. Thank you for being so kind and warm each time we spoke.

And thank you to my fellow women in science, who will forever have a place in my heart. Thank you Lindsay McAfee for joining me in South Florida during my first year here in Miami and being such an amazing and inspiring science educator. We have had so many adventures the past few years, from Oregon and California, to Florida and back to the west coast in Washington. Thank you for grounding me. Thank you to Whitney Leach, who quickly became a friend at SICB and has lent her support over this past year. Thank you to Nichole Tiernan for involving me in outreach and ensuring I was social during these past few years. Again, thank you to Dr. Angela Poole and Dr. Sheila Kitchen. For always being there to answer texts and chat whenever possible. I am so fortunate to have you in my life, to listen to my worries and to have a friend to sing karaoke alongside. My deepest gratitude for both of you fabulously stylish women who have taught me to go after what I want for myself.

To all of those along the way, thank you. This research was supported by NSFISO CAREER grant \#1453519 (to MRL) and NSF EDGE grant \#1645164 (to MRL, VMW, MD, AG, JP). 


\title{
ABSTRACT OF THE DISSERTATION \\ EVOLUTIONARY EXPANSIONS AND NEOFUNCTIONALIZATION OF IONOTROPIC GLUTAMATE RECEPTORS IN CNIDARIA
}

\author{
by \\ Ellen Grace Dow \\ Florida International University, 2019 \\ Miami, Florida

\section{Professor Mauricio Rodriguez-Lanetty, Major Professor}

Reef ecosystems are composed of a variety of organisms, transient species of fish and invertebrates, microscopic bacteria and viruses, and structural organisms that build the living foundation, coral. Sessile cnidarians, corals and anemones, interpret dynamic environments of organisms and abiotic factors through a molecular interface. Recognition of foreign molecules occurs through innate immunity via receptors identifying conserved molecular patterns. Similarly, chemosensory receptors monitor the environment through specific ligands. Chemosensory receptors include ionotropic glutamate receptors (iGluRs), transmembrane ion channels involved in chemical sensing and neural signal transduction. Recently, an iGluR homolog was implicated in cnidarian immunological resistance to recurrent infections of bacterial pathogens. I postulate that iGluRs in cnidarians may act as danger-sensing and/or pathogen recognition receptors adjacent to immune defense and nervous system signaling. In Chapter One, I explain the exploration of diversity and divergence within cnidarian iGluRs, complimented with predicted functions in the context of correlated response to biological and environmental signals, setting the groundwork for functional characterization. In Chapter Two, I characterized 
the divergence of cnidarian iGluRs in comparison to other metazoans through maximum likelihood phylogenetic analyses, which revealed greater evolutionary expansion of cnidarian iGluR lineages, including a Cnidaria-specific class. Gene expression differentiation implies select iGluRs respond transcriptionally to bacterial challenge, supporting the hypothesis that cnidarian iGluRs respond to pathogen signals. In Chapter Three, I investigated a putative endogenous rhythm to iGluR expression, as chemosensory receptors may have the capacity to anticipate daily environmental fluctuations. While a circadian rhythm does not appear to be a primary contributor to biological rhythms in iGluR gene expression, symbiosis and diurnal fluctuations are implicated factors. In Chapter Four, I chromogenically localized Exaiptasia pallida iGluR expression to the epidermis and concentrated within sensory tentacles, alongside cnidocytes. Expression of iGluRs in proximity of sensory cells is consistent with the putative function of iGluRs in cnidarian neural signaling. In the final chapter, I synthesized my research in its entirety; highlighting that cnidarian iGluRs expansions indicate cnidarian-specific neofunctionalization towards functions of chemosensory cnidarian-environmental signaling. New hypotheses and future research are presented to continue the study of iGluRs as chemosensory receptors within the cnidarian nervous system. 


\section{TABLE OF CONTENTS}

CHAPTER

PAGE

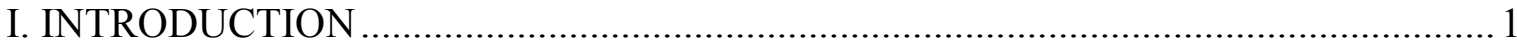

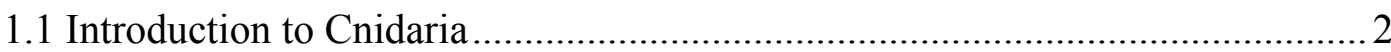

1.2 Innate immunity within cnidarians and the importance of pattern

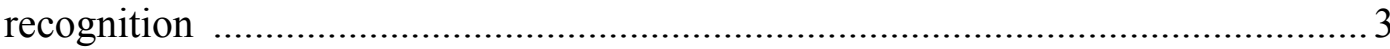

1.3 Chemosensation as a proposed signaling network within anemones ................. 5

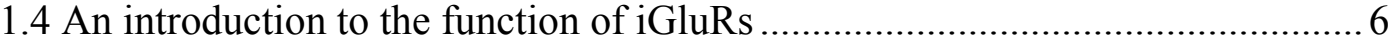

$1.5 \mathrm{~A}$ brief review of the dispersed nervous system and defensive cells in

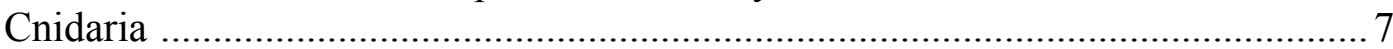

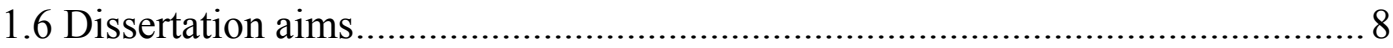

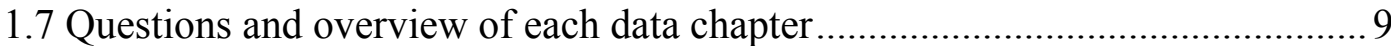

1.8 Relevance and significance of cnidarian iGluRs ................................................ 11

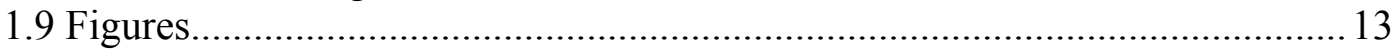

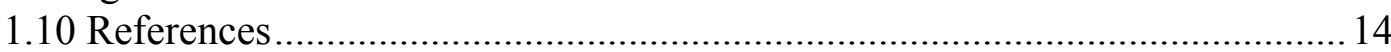

II. CNIDARIAN-SPECIFIC EXPANSION OF IONOTROPIC GLUTAMATE RECEPTORS PROMOTE NEOFUNCTIONALIZATION WITH IMPLICATIONS

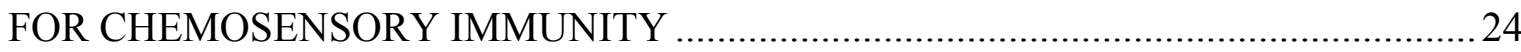

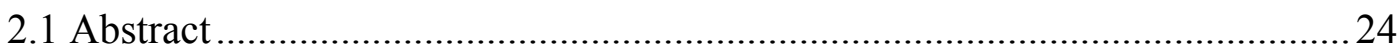

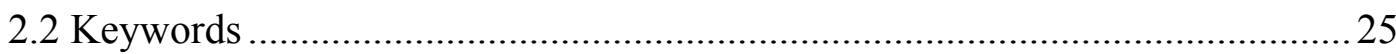

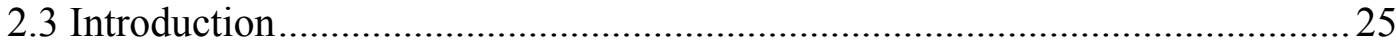

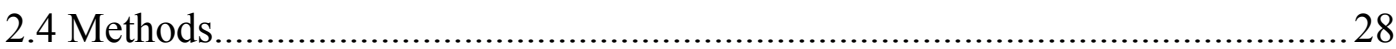

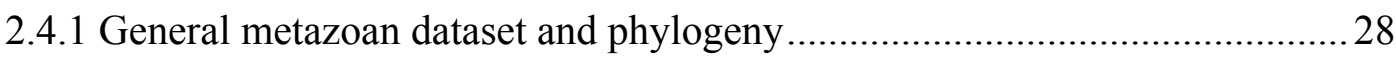

2.4.2 Cnidarian-specific dataset and phylogeny ..............................................2

2.4.3 Modeling the molecular structure of GluRs for functional insights ..............29

2.4.4 Investigation of Exaiptasia iGluRs transcriptional expression under

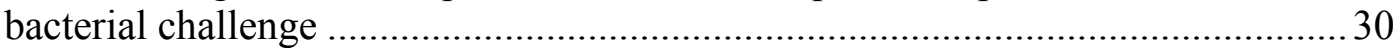

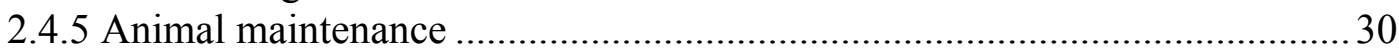

2.4.6 Culturing bacteria (Vibrio coralliilyticus and Serratia marcescens) and

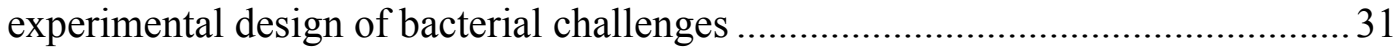

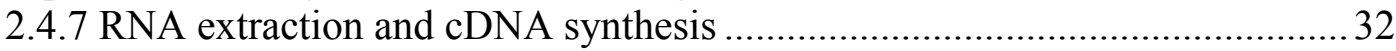

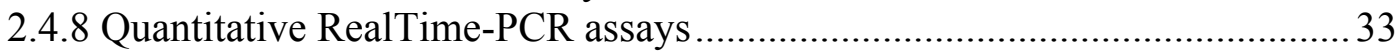

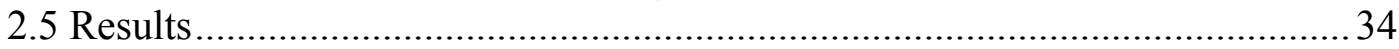

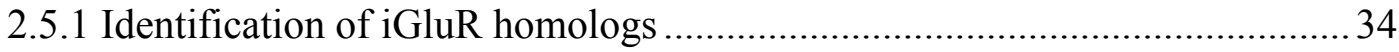

2.5.2 Phylogenetic inferences of cnidarian iGluRs to representative iGluR

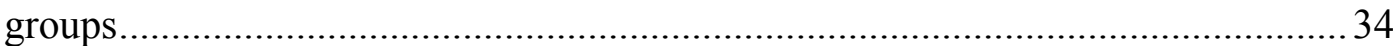

2.5.3 Molecular modeling of Exaiptasia pallida iGluRs with predictive

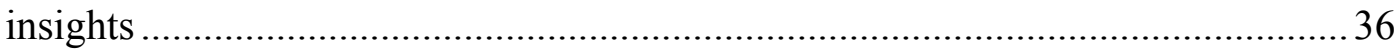

2.5.4 Transcriptional analysis of iGluR gene expression following bacterial challenge 


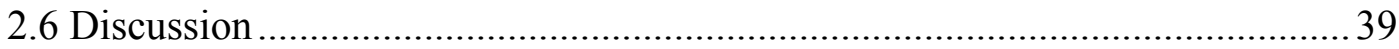

2.6.1 Evolutionary expansions of iGluRs in Cnidaria ……………….....................39

2.6.2 Neofunctionalization and subfuctionalization of cnidarian iGluRs................4 41

2.6.3 Certain iGluR clades in Exaiptasia pallida are functionally implicated

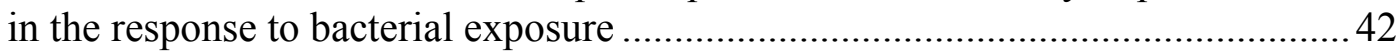

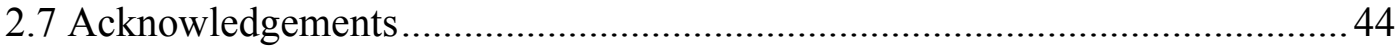

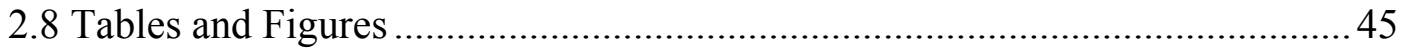

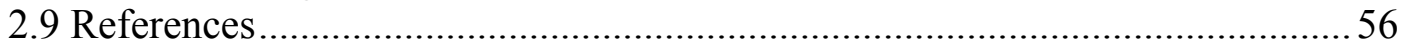

\section{EXPRESSION OF CHEMOSENSORY IONOTROPIC GLUTAMATE}

RECEPTORS UNDER THE INFLUENCE OF BIOLOGICAL AND

ENVIRONMENTAL OSCILLATIONS IN CNIDARIANS .............................................63

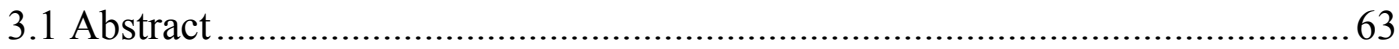

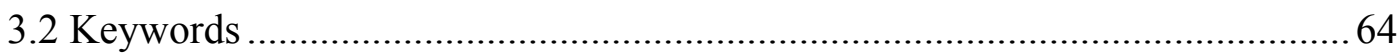

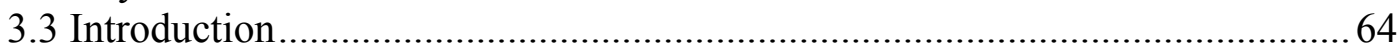

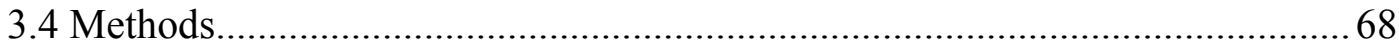

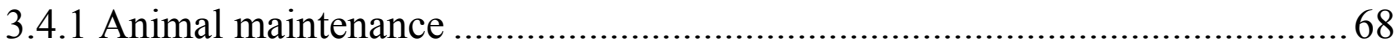

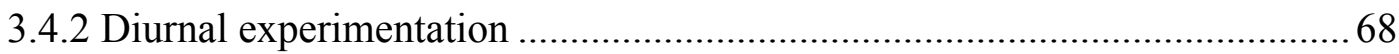

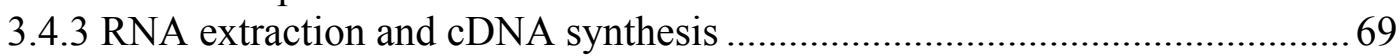

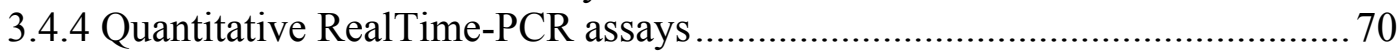

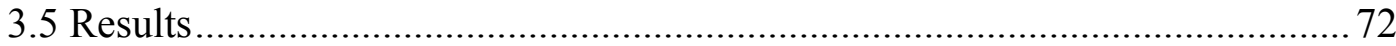

3.5.1 qPCR assays of Exaiptasia pallida gluR2 under light treatments ..................72

3.5.2 qPCR assays of Exaiptasia pallida gluR8 under light treatments ...................73

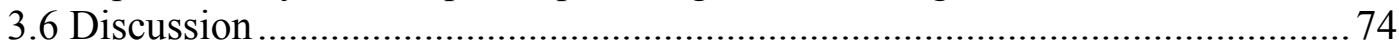

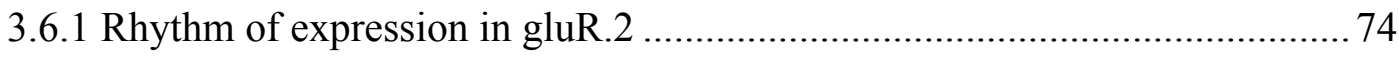

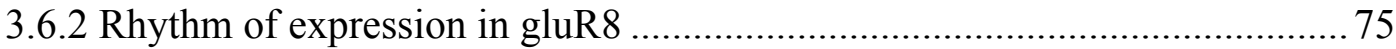

3.6.3 Robustness of detecting host rhythms......................................................... 75

3.6.4 Expression of iGluRs in Exaiptasia diaphana from transcriptomic data published by Sorek et al. 2018 .......................................................................... 76

3.6.5 Symbiodiniaceae and light influences on iGluR expression rhythms ............77

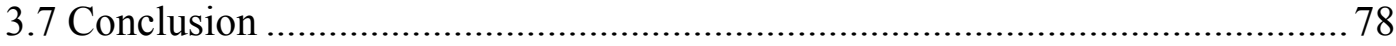

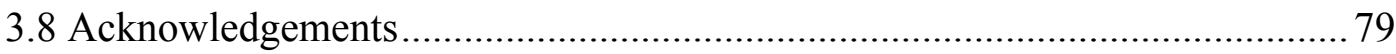

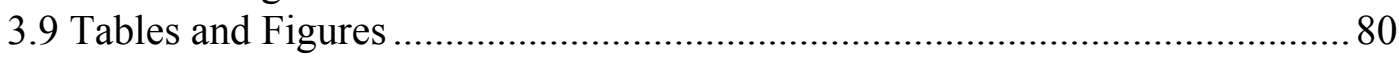

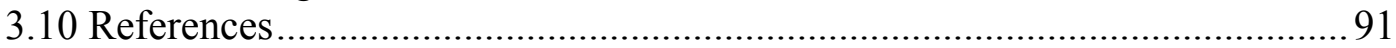

IV. LOCALIZATION OF IONOTROPIC GLUTAMATE RECEPTORS WITHIN THE CNIDARIAN EXAIPTASIA PALLIDA VIA IN SITU HYBRIDIZATION................98

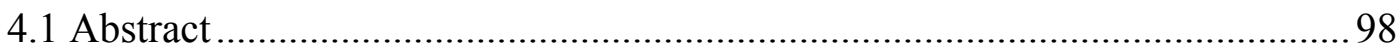

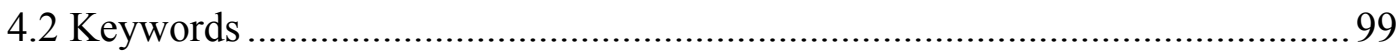

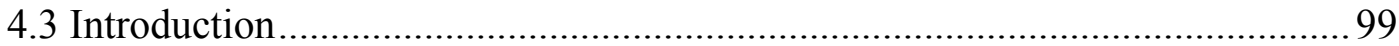

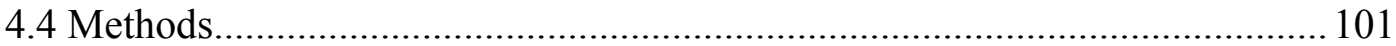

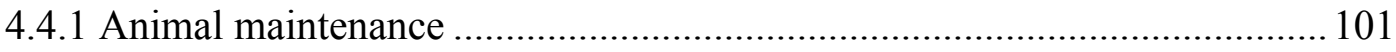

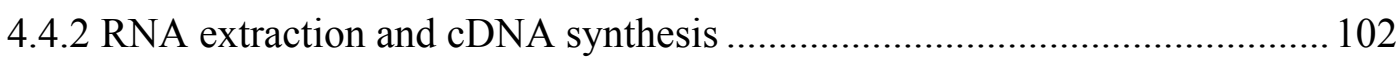

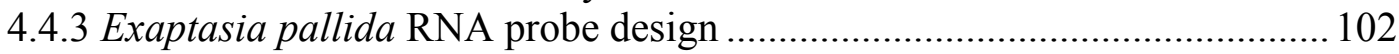

4.4.4 Chromogenic RNA in situ hydridization ..................................................... 104 


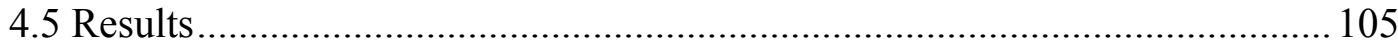

4.5.1 Identifiable anatomy of whole mount Exaiptasia pallida anemones............ 105

4.5.2 Localization of gluR genes in whole mount Exaiptasia pallida ................... 106

4.5.3 RNA localization of gluRs in relation to sensory cells ................................ 108

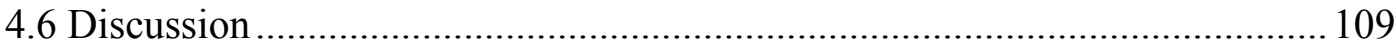

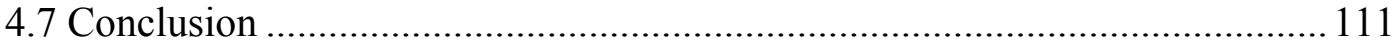

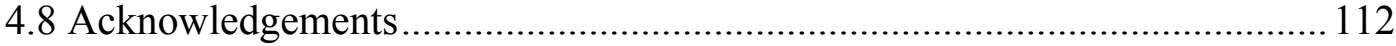

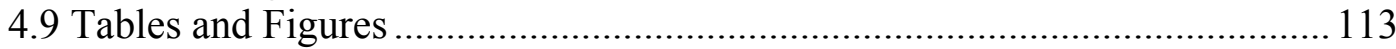

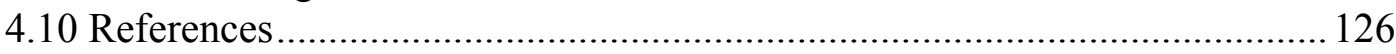

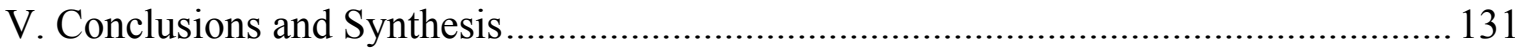

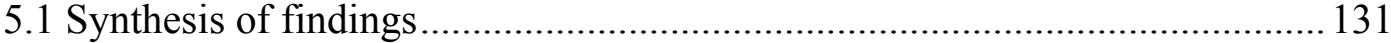

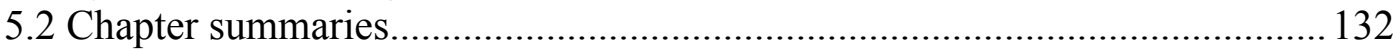

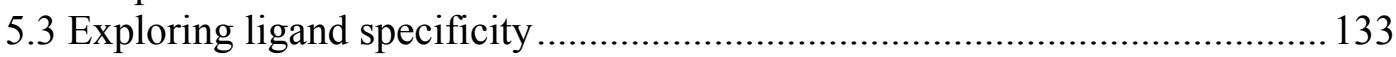

5.4 Putative functions of Exaiptasia pallida iGluRs ............................................ 135

5.5 Chromogenic localization of gluRs within Exaiptasia pallida ........................ 137

5.6 Ecological and applicable relevance of cnidarian iGluRs ................................ 139

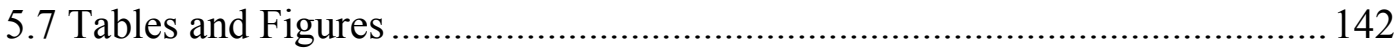

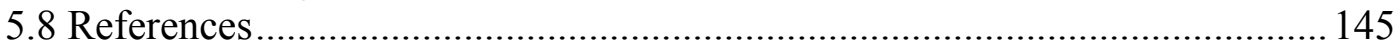

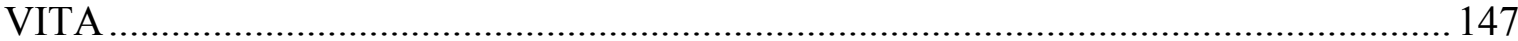




\section{LIST OF TABLES}

CHAPTER-TABLE

PAGE

2.S1 Taxa included within analysis and abbreviations used along with the number of sequences per taxa included in the iGluR Phylogenies for both cnidarian and general metazoan trees

2.S2 Primer sequences with names and gene identification.

2.S3 Statistical significance of 2-way ANOVA between factors of time and treatment

2.S4 Statistical significance of 2-way ANOVA Tukey HSD post-hoc using 95\% family-wise confidence level on treatment factor. 48

3.1 Primer sequences for qPCR assay with sequence names and gene identity 80

3.2 Two-way ANOVA p-values for factors and significant time points of Exaiptasia pallida gluR. 2 and gluR8 gene expression.

3.3 Statistical outputs of Jonckheere-Terpstra-Kendall analysis for Exaiptasia pallida gluR mRNA expression values.

3.4 iGluR homologs and related genes with biological rhythms identified in Exaiptasia diaphana in symbiotic (sym) and aposymbiotic (apo) states.

4.1 Coding sequence primers for Exaiptasia pallida gluR cloning

4.2 Exaiptasia pallida gluR primers with pGEMHE overhangs for directional assembly.....

4.3 Summary of Exaiptasia pallida gluR localization patterns of gene expression and representation across biological replicates.

5.1 Overview of Exaiptasia pallida iGluR characterization and findings.

5.2 Summary of questions and hypotheses proposed in the dissertation and concluding statements. 


\section{LIST OF FIGURES}

1.1 Infographic on the putative nature of iGluRs within the sea anemone Exaiptasia pallida.

2.1 Maximum likelihood phylogenetic tree based on amino acid sequences of iGluR homologs from cnidarian species and NMDA-specific iGluRs (GluN).

2.2 Conserved domains of Exaiptasia pallida ionotropic glutamate receptors..

2.3 Homotetramers of Exaiptasia pallida GluR6 and GluR.1

2.4 Relative fold difference ( $\log 2$ scale) in gene expression of E. pallida gluR variants upon treatment with bacterial challenges (Vibrio coralliilyticus and Serratia marcescens) across time.

2.5 Proposed evolutionary tree for iGluR subfamily Epsilon to include cnidarianspecific divergent clade.

2.S1 Maximum likelihood phylogenetic tree based on amino acid sequences of iGluR homologs from Exaiptasia pallida and reference proteomes

2.S2 Experimental set up of bacteria challenges on Exaiptasia pallida as described in Methods

3.1 Preliminary data of relative gene expression of Exaiptasia pallida gluR variants across time 84

3.2 Representation of glutamate concentration cycling in cnidarians. 85

3.3 Experimental design and set-up of the light treatment cycles for Exaiptasia pallida anemones 86

3.4 Boxplots of Exaiptasia pallida gluR.2 normalized relative quantities under $12 \mathrm{hr}$ dark: $12 \mathrm{hr}$ light (LD) and constant darkness (DD) over 24 hours.

3.5 Boxplots of Exaiptasia pallida gluR8 normalized relative quantities under $12 \mathrm{hr}$ dark: $12 \mathrm{hr}$ light (LD) and constant darkness (DD) over 24 hours. 88

3.6 Relative expression of iGluR-related contigs with 12-hr periodicity identified in Exaiptasia diaphana to compare symbiotic and aposymbiotic rhythm patterns. 
3.7 Relative expression of iGluR-related contigs with 24-hr periodicity identified in Exaiptasia diaphana to compare symbiotic and aposymbiotic rhythm patterns.

4.1 Cell differentiation and anatomy of Exaiptasia pallida anemones

4.2 Whole mount in situ hybridization of Exaiptasia pallida anemones for gluR expression localization at $100 \mathrm{X}$ magnification under $70 \mathrm{X}$ camera magnification

4.3 Tentacles of whole mount in situ hybridization of Exaiptasia pallida anemones for gluR expression localization at 1000X magnification under 70X camera magnification 118

4.4 Expression of Exaiptasia pallida gluR.1 on tentacles at 400x magnification 119

4.5 Pattern of Exaiptasia pallida gluR4 expression on tentacles. 120

4.6 Exaiptasia pallida gluR expression occuring at epidermal tissue layers. 121

4.7 Exaiptasia pallida gluR4 expression occuring at epidermal tissue layers on polyp column

4.8 Exaiptasia pallida gluR8 expression patterns on polyp column at 1000x magnification

4.9 Exaiptasia pallida gluR12 expression patterns on polyp column

4.10 Expression of Exaiptasia pallida gluRs on polyp column of whole mount anemones

5.1 Graphic of hypothesis depicting bacterial and diurnal factors that affect Exaiptasia pallida iGluR expression 


\section{ABBREVIATIONS AND ACRONYMS}

AMPA alpha-amino-3-hydroxy-5-methyl-4-isoxazolepropionic acid

ANOVA analysis of variance

ATD amino terminal domain (also known as NTD as N refers to any amino acid)

BLAST basic local alignment tool

C1 phylogenetic clade 1 from Fig. 2.1 and 2.S1

C2 phylogenetic clade 2 from Fig. 2.1 and 2.S1

C3 phylogenetic clade 3 from Fig. 2.1 and 2.S1

C4 phylogenetic clade 4 from Fig. 2.1 and 2.S1

C5 phylogenetic clade 5 from Fig. 2.S1, exclusive to IRs

C6 phylogenetic clade 6 from Fig. 2.S1, exclusive to GluD

C7 phylogenetic clade 7 from Fig. 2.S1, exclusive to Trichoplax adhaerens (Placozoa) sequences

C8 phylogenetic clade 8 from Fig. 2.S1, exclusive to GluK

CNS central nervous system

CX phylogenetic clade 1 from Fig. 2.1, exclusively Mnemiopsis leidyi (Ctenophora) sequences

DAMP damage-associated molecular pattern

DD $12 \mathrm{hr}$ dark : $12 \mathrm{hr}$ dark diurnal cycle, also constant darkness

EtOH ethanol

FISH fluorescent in situ hybridization 


\begin{tabular}{|c|c|}
\hline GluA & AMPA-specific ionotropic glutamate receptor, protein \\
\hline GluD & ionotropic glutamate receptor delta, protein \\
\hline GluK & kainate-specific ionotropic glutamate receptor, protein \\
\hline GluN & NMDA-specific ionotropic glutamate receptor, protein \\
\hline GluR & refers protein sequence of $E$. pallida iGluR subunit \\
\hline gluR & refers to gene sequence of $E$. pallida $\mathrm{iGluR}$ \\
\hline GR & gustatory receptor \\
\hline HSD & honestly significant difference, associated with Tukey post-hoc \\
\hline iGluR & ionotropic glutamate receptor \\
\hline IL-1R & interleukin-1 receptor \\
\hline IR & ionotropic receptor \\
\hline ISH & in situ hybridization \\
\hline LBD & ligand binding domain \\
\hline LD & $12 \mathrm{hr}$ light : $12 \mathrm{hr}$ dark diurnal cycle \\
\hline MAMP & microbe-associated molecular pattern \\
\hline ML & maximum likelihood phylogenetic analysis \\
\hline NMDA & N-methyl-D-aspartic acid \\
\hline NTD & see ATD \\
\hline OR & olfactory receptor \\
\hline
\end{tabular}




$\begin{array}{ll}\text { PAMP } & \text { pathogen-associated molecular pattern } \\ \text { PCR } & \text { polymerase chain reaction } \\ \text { PRR } & \text { pattern recognition receptor } \\ \text { qPCR } & \text { quantitative polymerase reaction (also RT-qPCR) } \\ \text { RNA } & \text { ribonucleic acid } \\ \text { TIR } & \text { toll/interleukin-1 receptor } \\ \text { TLR } & \text { toll-like receptor } \\ \text { TMD } & \text { transmembrane domain }\end{array}$




\section{CHAPTER I. INTRODUCTION}

Organisms sense immediate environments through an interface of essential barriers and receptors designated to defend against harmful infectious agents. Immune systems differentiate between self and non-self (1), which is observed from microscopic singleliving prokaryotes i.e., bacteria retaining innate immune mechanisms recognizing viral infections $(2,3)$ to complex multicellular organisms harboring additional learning-based recognition pathways $(4,5)$. There are two primary systems immunity relies upon, innate and adaptive. Innate immunity has evolved across pathways for organisms to inherently recognize foreign entities through a genome-based system $(4,6-8)$. Adaptive immunity prolongs specific resistance against pathogens over longer periods; however, evidence of adaptive immunity is not observed in early-diverged metazoans (8-11). Current findings support the theory of simultaneous evolution of innate and adaptive immune systems, rather than a previous interpretation that put forth expanding adaptive immunity upon the foundation of an innate system (5). The observation of conserved gene sequences suggests each pathway has independently evolved across phyla. Invertebrates primarily rely on innate functions, coded within genomes, for identifying microbial and/or chemical cues to distinguish pathogenic agents $(8,11-14)$. The growing availability and understanding of '-omics' data in early-diverged metazoans allows for gaps to be filled within complexities of innate immunity, one group of particular interest is Cnidaria. 


\subsection{Introduction to Cnidaria}

Cnidarians are named by their distinctive stinging cells referred to as cnidocytes, which are used in defense and prey capture (15-18). The importance of accumulating genetic data from cnidarians comes from the ecological importance of reef-building corals that form the highly productive framework of diversified reef ecosystems, which provides essential habitats for fish and marine invertebrates (19-22). Reefs are known for a number of canonical symbioses. In general, symbiosis between diverse organisms provide the basis for fitness success across environments (23-25). Corals provide habitat for microorganisms, creating specific microbiomes for different species and habitat ranges (26-28). A unique endosymbiosis that has driven molecular and signaling research within Cnidaria occurs between cnidarian hosts and photosynthetic algal dinoflagellates from the family Symbiodiniaceae (29-38). The cnidarian-Symbiodiniacea mutualism is

the main source of primary productivity for coral reef habitats in tropical and subtropical regions (38-41). The host cell provides dissolved inorganic carbon, nitrates, phosphates, and essential compounds to endosymbiotic Symbiodiniaceae in exchange for organic carbon and nitrates from metabolic output of dinoflagellates $(33,35,37,42-47)$. Immune regulation is hypothesized to serve for continuation of winnowing, or selecting and filtering specific symbionts for mutualism (48-50).

Cnidarian hosts differentiate pathogenic and mutualistic symbionts using various signaling pathways. Signaling and receptor pathways have been outlined in Cnidaria predominantly by identifying homologs of established proteins from model systems within cnidarian genomes and transcriptomes. Sequenced cnidarian genomes include 
Hydra magnipapillata, a hydrozoan, and Nematostella vectensis, an aposymbiotic anthozoan, Acropora digitifera and Stylophora pistllata, scleractinian corals, and symbiotic and aposymbiotic of Exaiptasia pallida (colloquially referred to as Aiptasia) (51-56). Additional transcriptomes have been established along with these genomes, to identify various homologous innate immune genes between cnidarians and higher metazoan systems (57-61).

\subsection{Innate immunity within cnidarians and the importance of pattern recognition}

The primary knowledge of innate immunity in cnidarians is based on reception, followed by predicted pathways from genetic data. Recognition occurs in cnidarians through detection of microbial- or pathogen-associated molecular patterns (MAMPs or PAMPs) by pattern recognition receptors (PRRs) as a function of innate immunity $(7,50,56,59,62,63)$. The group of PRRs identified in early-divergent metazoans includes Toll-like receptors (TLRs) and related Toll/Interleukin-1 Receptors (TIR domains) located within sponges (64) and cnidarians (65-67). The TLR signaling pathway in the anemone Nematostella vectensis follows a bilaterian metazoan path, including the presence of an intracellular TIR domain connected to extracellular LRRs $(8,67)$. The occurrence of five TIR-containing proteins in $N$. vectensis (13), three of which contain multiple immunoglobin domains and resemble mammalian interleukin-1 receptors (IL1Rs) (68), is consistent with the hypothesized development of the TLR pathway prior to the divergence of cnidarians. Furthermore, immune genes identified in Hydra, include four TIR-domain-containing proteins of which two have similar features to MyD88, including a death domain, and two TIR homologs lacking LRRs (68-71). The TLR 
signaling pathway appears to be essential for recognizing different microbes in Cnidaria, as suggested from evidence of differential host gene expression, altered bacterial colonization, and susceptibility to pathogens within MyD88-knockdown Hydra polyps $(72,73)$. The MyD88-mediated TLR signaling cascade in Hydra does not appear to follow the canonical bilaterian pathway, while it is hypothesized to be have a role in establishing bacteria colonies (72). However, substantial gene-loss may have occurred between hydrozoans and cnidarians, due presence of canonical Toll/TLR pathway in cnidarians, but lack thereof in hydrozoans $(65,72)$. Gene-loss within cnidarian innate immunity appears to happen randomly within immune pathways through a loss of Toll, TLR, and IL-1R receptors in Hydra, while pathway intermediates remain present (Miller et al., 2007). This brings to the forefront the importance of identifying alternative immune or signaling pathways within Cnidaria.

Prospective signaling pathways and organismal systems may have crucial involvement in innate immunity as regulators during the early stages of the onset of symbiosis or pathogen response, as microbial infections activate transcriptional activity in immune pathways $(74,75)$. Pathways that use receptors similar to PRRs may have a higher likelihood to identify infectious agents because of conservation across bacteria and other signaling pathways. Potentially, chemical signals could be used as a molecular pattern for receptors similar to PRRs, such as chemosensory proteins. A family of chemosensory proteins has been correlated to immune response through up-regulation in response to coral pathogenic bacterium Vibrio coralliilyticus (76), implying 
environmental chemical signals could serve in cnidarian recognition of conserved chemical patterns and ligands.

\subsection{Chemosensation as a proposed signaling network within anemones}

Chemosensing is defined as the transduction of a chemical signal by a receptor, tangential to the nervous system and built upon behaviors leading to a biological response $(77,78)$. Organisms require continuous input of sensory information for behaviors that contribute to acquiring resources for survival and reproduction $(79,80)$, which includes receiving chemical signals to interpret the environment. Chemosensory receptors, such as ionotropic glutamate receptors (iGluRs), within invertebrates distinguish changes in the environment and send consequent signals through the nervous system $(79,81)$. Within the cnidarian Exaiptasia pallida, an iGluR protein fragment was found to be up-regulated post-exposure to $V$. coralliilyticus (76).

Ionotropic glutamate receptors recognize glutamate or a receptor-specific ligand in the receptor domain, which opens the transmembrane channel to transport calcium ions into the cytoplasm of the cell and elicit consequent physiological responses $(80,82)$.

Receptor cells will outnumber central neurons responsible for processing sensory information within the nervous system, requiring many types of signals to interpret and distinguish various cues (79). Aside from iGluRs, other chemoreceptors i.e., odor receptors (ORs) and gustatory receptors (GRs), may offer cnidarians an additional path to recognize external chemical cues $(77,83-87)$. These receptors aid in prey capture, predator evasion and other roles essential to survivorship (77). The use of chemosensing 
in early-diverging metazoans (88) and Caenorhabditis elegans (89) is linked to behavior patterns, as any response to an external stimulus has the potential to trigger a conscious or unconscious physiological and/or behavioral change. The chemosensory response begins with a receptor with ligand specificity, similar to PRRs.

\subsection{An introduction to the function of iGluRs}

The family of iGluRs are receptors known for neuronal communication in sensory responses to environmental factors $(77,80,88,90,91)$. However, iGluRs are conserved across metazoans and within plants. In Arabidopsis thaliana, iGluRs are responsible for root signaling and implicated in the danger theory response to tissue damage accrued by the presence of a pathogen (92-98). Expanding into metazoans, iGluRs are present within the sponge phyla, Porifera, and are implied to have a role in sponge environmental sensing $(14,99)$. In Ctenophora, iGluRs are important in signaling and development of the uniquely complex nervous systems of comb jellies $(87,100,101)$, while the role of iGluRs within bilaterian invertebrates expands to chemosensing. A flavor of iGluRs, known as Ionotropic Receptors (IRs) have an important role in olfaction and identifying food sources in insects $(77,88,102)$. Chemosensory organs characteristically have IRs localized to their specialized tissues within invertebrates, including insect antennae and mollusk rhinophores $(77,79,103)$. Within vertebrate systems, iGluRs are responsible for synapses within the central nervous system (CNS) particularly receiving signals within the brain $(81,103-105)$. The consequence of iGluRs is clear in deregulation of glutamate leading to neurodegeneration $(106,107)$. Conversely, in cnidarians the role and extent of diversity of iGluRs is unknown, however there presence has been observed $(77,108)$. 


\subsection{A brief review of the dispersed nervous system and defensive cells in Cnidaria}

Cnidarians do not possess a CNS that would traditionally interpret iGluR signals. Rather, cnidarians have a nerve net, or diffused nervous system composed of sensory and ganglionic neurons, and their respective processes interspaced among epithelial cells $(87,109,110)$. The nervous system present in anthozoans, such as the non-symbiotic anemone Nematostella vectensis, is composed of linear tracts of neurites, or a nerve cord, within the parietal region and between mesenterial endomesoderms $(78,85,111)$. Directional neural conduction triggers local muscle contraction during neuromyoepithelial synapses $(78,111-113)$. The nervous system of sensory cells and neurons are interwoven throughout the epidermis to connect with mechanical and chemical defense mechanisms.

Cnidarian tentacles concentrated with defensive stinging cells of nematocytes and spirocytes, have microscopic harpoons called nematocysts and spirocysts, respectively (114-117). Synaptic modulation controls by spirocyst discharge by mutliple neurotransmitters $(85,114,118)$. Glutamate, a known neurotransmitter is known to aid in triggering a response to prey and self-defense $(85,115,117)$ In non-symbiotic Hydra, iGluRs modulate signal coordination and increase of nematocyst launching or discharge (85). The connection between iGluRs with and bacteria is implied the presence of polygamma-glutamic acid that could trigger nematocyst discharge (117), which is also consistent with the hypothesis of iGluR involvement in defensive chemosensory pathways. While initial theories postulated mechanosensory stimulus, current findings 
suggest chemosensory-triggered discharge of nematocytes opposed to solely physical contact (115). The correlation of iGluRs involved in signaling from the perspective of neurosensory system within Cnidaria to the cnidocyte glutamate response; connecting immunity and nervous system through fast signaling.

\subsection{Dissertation aims}

Recent evidence of iGluRs in anthozoans comes from E. pallida from an iGluR protein sequence identified as up-regulated in response to a bacterial challenge (76). Cnidarian iGluRs are implicated in putative chemosensory and/or immune responses to bacteria, therefore I used E. pallida as a model system $(56,59)$ to understand the role of iGluRs in cnidarians and their potential as chemoreceptors correlated with innate immunity (Fig. 1). I used several methods to begin characterizing the iGluR protein family within Cnidaria. In Chapter Two, I used a phylogenetics approach to determine the extent of cnidarian diversity and homolog conservation as a starting point for predicting putative functions. The available genome and transcriptome of E. pallida was important in the molecular framework for measuring transcriptional expression. Analyzing the response of a model lab cnidarian, a sea anemone to bacteria challenges and across diel treatments as an introduction to correlating iGluR response to the environment within Chapters Two and Three. Chromogenic localization of gene expression within Chapter Four was then used to lay the groundwork for dispersal of expression among cells, particularly in relation to sensory cells and the nervous system. 


\subsection{Questions and overview of each data chapter}

\section{Chapter II}

- What is the extent of cnidarian iGluR diversity?

- Are the protein structures of E. pallida iGluRs reflective of functional characteristics through conserved domains?

- What level are iGluRs involved during the response to microbial challenges?

The aim of this first data chapter was to determine the extent of cnidarian iGluR diversity and propose putative functions for iGluRs within E. pallida. As few iGluRs have been noted or compared to model species iGluRs $(77,108,119,120)$, the necessity to explore homolog diversity and begin exploration into putative functions was the first step in characterizing cnidarian iGluRs. Through comparing reference proteomes (defined by Uniprot as a subset of proteomes selected following criteria to represent model organisms and species of interest) and predicted proteomes from Reef Genomics database, I traced cnidarian iGluRs among other metazoan lineages of classically described iGluRs. From the lineage specific expansions of iGluRs within Exaiptasia pallida, the conserved domains were compared to predict functions and the potential of functional protein units based on completeness. Bioinformatic inferences were coupled with correlative analysis

of the transcriptional response of iGluRs to pathogenic bacterial challenges within the lab model cnidarian E. pallida. The reasoning behind the initial approach was the identification of an iGluR peptide fragment as highly up-regulated in response to a pathogenic challenge to Vibrio coralliilyticus $(76,121)$. 


\section{Chapter III}

- Is there a daily expression cycle for cnidarian iGluRs?

- Do cnidarian iGluRs have a role in biological cycles?

The aim of the second data chapter was a continuation of the first predictive exploration of iGluRs within E. pallida. The transcriptional levels of iGluRs within E. pallida varied across time points, regardless of treatment and including the control anemones, thus a continuation into the gene expression of iGluRs within a 24-hr cycle was investigated. The purpose of this chapter was to explore the expression cycles of iGluRs in the perspective of biological rhythms using a temporally robust experiment with independent biological replicates. Analysis of this experiment continued beyond diel cues of light and into the presence of Symbiodiniaceae within another cnidarian species from prior published, but not addressed, transcriptomic data (122). Integrating contributions of diel and symbiotic factors to iGluR expression patterns lead to hypotheses proposing metabolites from Symbiodiniaceae putatively affect rhythms of iGluR expression.

\section{Chapter IV}

- Where are iGluRs localized within E. pallida?

The aim of the third data chapter was to localize iGluR expression within E. pallida using in situ hybridization. This is a significant contribution to the field, first through establishing a protocol for in situ hybridization within E. pallida that is robust and replicable, which can be used in further experiments within collaborating labs and the 
community at large, and second for genes with low expression. While the working hypothesis stated iGluRs were likely to be located in sensory tentacle cells and gastrodermal tissues that phagocytize pathogenic agents, the results indicated that iGluRs localized solely within the epidermis of anemones. Expression was concentrated within tentacles; however, some localization of iGluR expression had punctate patterns throughout the column of the anemone body plan. Localization of iGluRs has suggested future experiments for pursuing the function of cnidarian iGluRs.

\subsection{Relevance and significance of cnidarian iGluRs}

Current understanding of cnidarian immunity primarily relies on putative function extrapolations derived from homologs well characterized in other invertebrate or vertebrate model systems. The attention to understanding immune defense evolution $(8,13,123)$ remains biased toward vertebrate models with direct applications to human medicine (4,6-9). Pattern recognition receptors, components of the complement system, and alternative pathway are well-researched pathways known to contribute to innate immunity within cnidarians. However, there are knowledge gaps and unexplored signaling pathways within cnidarian immunity, including the role of chemosensory receptors.

Chemosensory proteins respond to specific ligands within the environment to evoke physiological changes and behaviors. One well-characterized chemosensory protein lineage within diverged metazoans is ionotropic glutamate receptors (iGluRs). Receptor proteins have diverse functions; known for receiving signals between synapses 
in vertebrate brain and serving sensory roles for food detection in flying insects. Putative iGluRs have yet to be characterized within cnidarians. Through characterizing cnidarian iGluRs within a model symbiotic cnidarian, E. pallida, current hypotheses of iGluR evolution will be challenged, having occurred earlier in evolutionary history than previously predicted. The current project utilized several molecular applications to explore the diversity of iGluR lineages within cnidarians and putative functions of cnidarian iGluRs within the context of response to environmental factors. The following chapters outline expression patterns of cnidarian iGluRs, which opens up the possibility of an interrelationship between neural signaling and innate immunity, and expands our understanding of the role of iGluRs in cnidarians. 


\subsection{Figures}

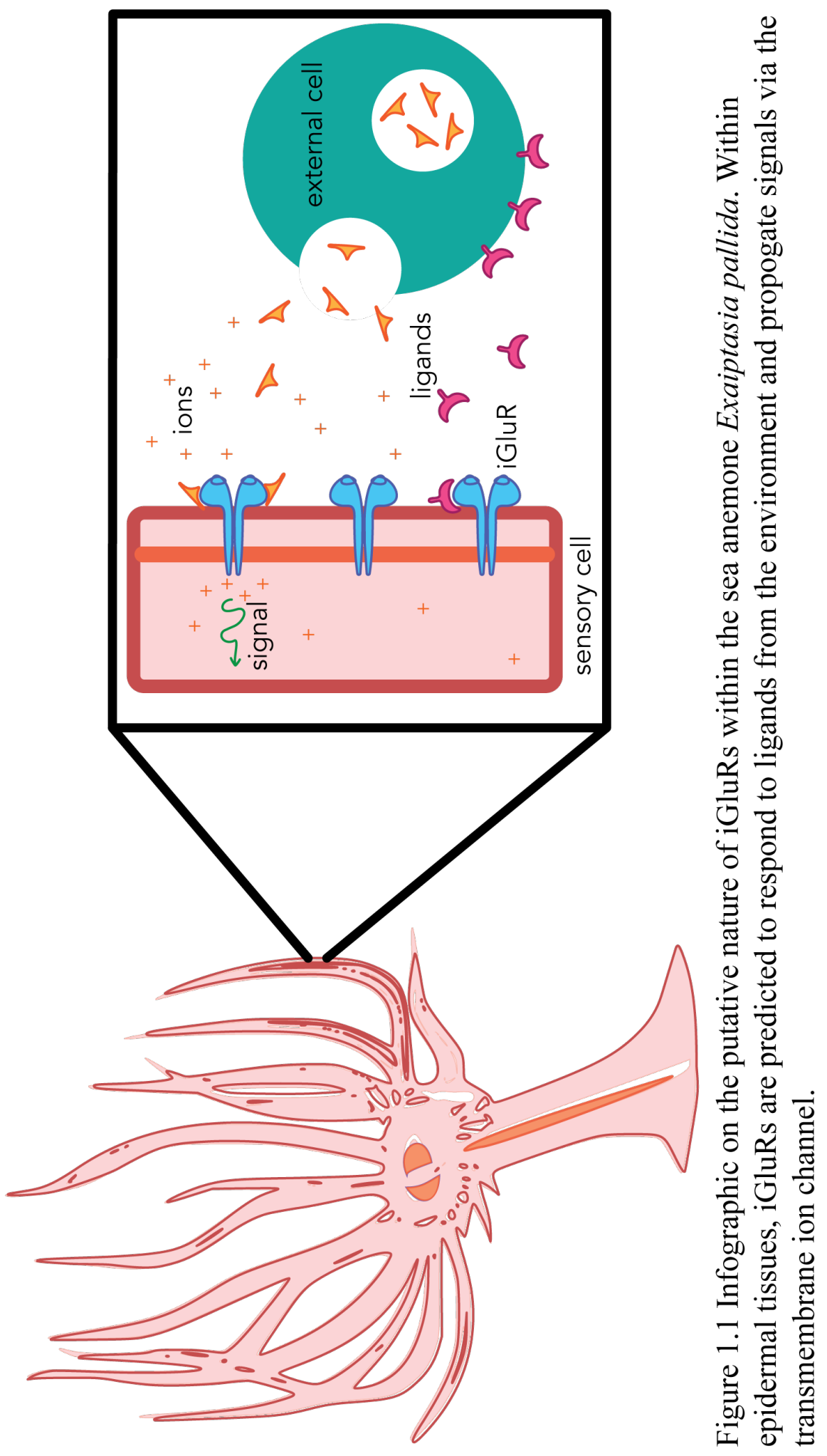




\subsection{References}

1. Nyholm S V, Graf J. Knowing your friends: invertebrate innate immunity fosters beneficial bacterial symbioses. Nat Rev Microbiol. 2012;10(12).

2. Seed KD, Lazinski DW, Calderwood SB, Camilli A. A bacteriophage encodes its own CRISPR/Cas adaptive response to evade host innate immunity. Nature. 2013;494(7438):489-91.

3. Seed KD. Battling Phages: How Bacteria Defend against Viral Attack. PLoS Pathog. 2015;11(6):e1004847.

4. Medzhitov R, Janeway CA. Innate Immunity: The Virtues of a Nonclonal System of Recognition. Cell. 1997;91:295-8.

5. Rimer J, Cohen IR, Friedman N. Prospects \& Overviews Do all creatures possess an acquired immune system of some sort? BioEssays. 2014;36:273-81.

6. Medzhitov R. Approaching the Asymptote: 20 Years Later. Immunity. 2009;30(6):766-75.

7. Beutler B. Innate immunity: an overview. Mol Immunol. 2004;40:845-59.

8. Hemmrich G, Miller DJ, Bosch TCG. The evolution of immunity : a low-life perspective. Trends Immunol. 2007;28(10).

9. Medzhitov R, Janeway Jr. CA. Innate immunity : impact on the adaptive immune response Ruslan Medzhitov and Charles A Janeway Jr. Curr Opin Immunol. 1997;9:4-9.

10. Janeway Jr. CA. Approaching the Asymptote? Evolution and Revolution in Immunology. Cold Spring Harb Symp Quant Biol. 1989;54:1-13.

11. Rinkevich B. The "immunology trap" of anthozoans. ISJ. 2011;8:153-61.

12. Little TJ, Hultmark D, Read AF. Invertebrate immunity and the limits of mechanistic immunology. Nat Immunol. 2005;6(7):651-4.

13. Miller DJ, Hemmrich G, Ball EE, Hayward DC, Khalturin K, Funayama N, et al. The innate immune repertoire in Cnidaria - ancestral complexity and stochastic gene loss. Genome Biol. 2007;8(4):R59. 
14. Degnan SM. The surprisingly complex immune gene repertoire of a simple sponge, exemplified by the NLR genes: A capacity for specificity? Dev Comp Immunol. 2015;48(2):269-74.

15. Sunagar K, Columbus-Shenkar YY, Fridrich A, Gutkovich N, Aharoni R, Moran Y. Cell type-specific expression profiling unravels the development and evolution of stinging cells in sea anemone. BMC Biol. 2018;16(1):1-16.

16. Babonis LS, Martindale MQ. Old cell, new trick? Cnidocytes as a model for the evolution of novelty. Integr Comp Biol. 2014;54(4):714-22.

17. Schuchert P. Phylogenetic analysis of the Cnidaria. J Zool Syst Evol Res. 2009;31(3):161-73.

18. Bosch TCG, Klimovich A, Domazet-Lošo T, Gründer S, Holstein TW, Jékely G, et al. Back to the Basics: Cnidarians Start to Fire. Trends Neurosci. 2017;40(2):92-105.

19. Weis VM. Cellular mechanisms of Cnidarian bleaching: stress causes the collapse of symbiosis. J Exp Biol. 2008;211:3059-66.

20. Oppen MJH Van, Oliver JK, Putnam HM, Gates RD. Building coral reef resilience through assisted evolution. PNAS Early Ed. 2015;1-7.

21. Barshis DJ, Ladner JT, Oliver TA, Seneca FO, Traylor-Knowles N, Palumbi SR. Genomic basis for coral resilience to climate change. Proc Natl Acad Sci U S A. $2013 ; 110(4)$.

22. Seneca FO, Palumbi SR. The role of transcriptome resilience in resistance of corals to bleaching. Mol Ecol. 2015;

23. Margulis L. Archaeal-eubacterial mergers in the origin of Eukarya: Phylogenetic classification of life. Proc Natl Accad Sci USA. 1996;93:1071-6.

24. Margulis L. Genetic and evolutionary consequences of symbiosis. Exp Parasitol. 1976;39(2):277-349.

25. Nyholm S, Stabb E V., Ruby EG, McFall-Ngai MJ. Establishment of an animalbacterial association: Recruiting symbiotic vibrios from the environment. PNAS. 2000;97(18):10231-5.

26. Rädecker N, Pogoreutz C, Voolstra CR, Wiedenmann J, Wild C. Nitrogen cycling in corals: The key to understanding holobiont functioning? Vol. 23, Trends in Microbiology. 2015. 
27. Blackall LL, Wilson B, Van Oppen MJH. Coral-the world's most diverse symbiotic ecosystem. Molecular Ecology. 2015.

28. Ainsworth TD, Krause L, Bridge T, Torda G, Raina J-B, Zakrzewski M, et al. The coral core microbiome identifies rare bacterial taxa as ubiquitous endosymbionts. ISME J. 2015;1-14.

29. Coffroth MA, Santos SR. Genetic diversity of symbiotic dinoflagellates in the genus Symbiodinium. Protist. 2005;156(1):19-34.

30. Fitt WK, Trench RK. Endocytosis of the Symbiotic Dinoflagellate Symbiodinium microadriaticum Freudenthal by Endodermal Cells of the Scyphistomae of Cassiopeia xamachana and Resistance of the Algae to Host Digestion. J Cell Sci. 1983;64:195-212.

31. LaJeunesse TC, Thornhill DJ. Improved resolution of reef-coral endosymbiont (Symbiodinium) species diversity, ecology, and evolution through psbA noncoding region genotyping. PLoS One. 2011;

32. LaJeunesse TC, Parkinson JE, Gabrielson PW, Jeong HJ, Reimer JD, Voolstra CR, et al. Systematic Revision of Symbiodiniaceae Highlights the Antiquity and Diversity of Coral Endosymbionts. Curr Biol. 2018;28(16):2570-2580.e6.

33. Muscatine L, Pool RR. Regulation of numbers of intracellular algae. Biol Sci Proc R Soc Lond B. 1979;204(204):131-9.

34. Muscatine L, Blackburn A, Gates RD, Baghdasarian G, Ferrier-Pagè C, Allemand $\cdot$ D, et al. Cell - specific density of symbiotic dinoflagellates in tropical anthozoans. Coral Reefs. 1998;17:329-227.

35. Trench RK. Dinoflagellates in non-parasitic symbioses. In: Biol Dinoflag. 1987. p. 530-70.

36. Blank RJ, Trench RK. Speciation and symbiotic dinoflagellates. Science (80- ). 1985;229(4714):656-8.

37. Muscatine L, Pool RR, Trench RK. Symbiosis of algae and invertebrates: aspects of the symbiont surface and the host-symbiont surface. Trans Am Microsc Soc. 1975;94(4):450-69.

38. Bucher M, Wolfowicz I, Voss PA, Hambleton EA, Guse A, Muscatine L, et al. Development and Symbiosis Establishment in the Cnidarian Endosymbiosis Model Aiptasia sp. Sci Rep. 2016 Jan 25;6(19867):19867. 
39. Reynolds WS, Schwarz JA, Weis VM. Symbiosis-enhanced gene expression in cnidarian-algal associations : cloning and characterization of a cDNA , sym32, encoding a possible cell adhesion protein. Comp Biochem Physiol Part A. 2000;126:33-44.

40. Ezzat L, Maguer J-F, Grover R, Ferrier-Pages C. New insights into carbon acquisition and exchanges within the coral- dinoflagellate symbiosis under $\mathrm{NH}_{4}{ }^{+}$ and $\mathrm{NO}_{3}{ }^{-}$supply. Proc R Soc London Ser B, Biol Sci. 2015;(282):20150610.

41. Little AF, van Oppen MJH, Willis BL. Flexibility in Algal Endosymbioses Shapes Growth in Reef Corals. Science (80- ). 2004;304(5676):1492-4.

42. Muscatine L. The role of symbiotic algae in carbon and energy flux in reef corals. Coral Reefs. 1990;(25):75-84.

43. Weis VM, Smith GJ, Muscatine L. A "CO2 supply" mechanism in zooxanthellate cnidarians: role of carbonic anhydrase. Mar Biol. 1989;100:195-202.

44. Yellowlees D, Rees TA V, Leggat W. Metabolic interactions between algal symbionts and invertebrate hosts. Plant, Cell Environ. 2008;31:679-94.

45. Ganot P, Moya A, Magnone V, Allemand D, Furla P, Sabourault C. Adaptations to endosymbiosis in a Cnidarian-Dinoflagellate association: Differential gene expression and specific gene duplications. PLoS Genet. 2011;7(7):e1002187.

46. Leal MC, Hoadley K, Pettay DT, Grajales A, Calado R, Warner ME. Symbiont type influences trophic plasticity of a model cnidarian-dinoflagellate symbiosis. J Exp Biol. 2015;218.

47. Trench RK. The Cell Biology of Plant-Animal Symbiosis. Annu Rev Plant Physiol. 1979;30:485-531.

48. Weis VM, Davy SK, Hoegh-Guldberg O, Rodriguez-Lanetty M, Pringle JR. Cell biology in model systems as the key to understanding corals. Trends Ecol Evol. 2008;23(7):369-76.

49. Sproles AE, Kirk NL, Kitchen SA, Oakley CA, Grossman AR, Weis VM, et al. Phylogenetic characterization of transporter proteins in the cnidariandinoflagellate symbiosis. Mol Phylogenet Evol. 2018;120:307-20.

50. Davy SK, Allemand D, Weis VM. Cell Biology of Cnidarian-Dinoflagellate Symbiosis. Microbiol Mol Biol Rev. 2012;76(2):229-61.

51. Chapman JA, Kirkness EF, Simakov O, Hampson SE, Mitros T, Weinmaier T, et al. The dynamic genome of Hydra. Nature. 2010;464(7288):592-6. 
52. Voigt O, Erpenbeck D, Wörheide G. A fragmented metazoan organellar genome: The two mitochondrial chromosomes of Hydra magnipapillata. BMC Genomics. 2008;9:1-10.

53. Shinzato C, Mungpakdee S, Satoh N, Shoguchi E. A genomic approach to coraldinoflagellate symbiosis: Studies of Acropora digitifera and Symbiodinium minutum. Front Microbiol. 2014;5(JULY):1-17.

54. Shinzato C, Shoguchi E, Kawashima T, Hamada M, Hisata K, Tanaka M, et al. Using the Acropora digitifera genome to understand coral responses to environmental change. Nature. 2011;476(7360):320-3.

55. Voolstra CR, Li Y, Liew YJ, Baumgarten S, Zoccola D, Flot JF, et al. Comparative analysis of the genomes of Stylophora pistillata and Acropora digitifera provides evidence for extensive differences between species of corals. Sci Rep. 2017;7(1):1-14.

56. Baumgarten S, Simakov O, Esherick LY, Liew YJ, Lehnert EM, Michell CT, et al. The genome of Aiptasia, a sea anemone model for coral symbiosis. Proc Natl Acad Sci. 2015;Early Edit.

57. Kitchen SA, Crowder CM, Poole AZ, Weis VM, Meyer E. De Novo Assembly and Characterization of Four Anthozoan (Phylum Cnidaria) Transcriptomes. G3 Genes Genomes Genet. 2015;5:2441-52.

58. Li B, Dewey CN. RSEM: accurate transcript quantification from RNA-Seq data with or without a reference genome. BMC Bioinformatics. 2011;12(323).

59. Lehnert EM, Burriesci MS, Pringle JR. Developing the anemone Aiptasia as a tractable model for cnidarian-dinoflagellate symbiosis: the transcriptome of aposymbiotic $A$. pallida. BMC Genomics. 2012;13:271.

60. Huang C, Leng D, Sun S, Zhang XD. Re-analysis of the coral Acropora digitifera transcriptome reveals a complex lncRNAs-mRNAs interaction network implicated in Symbiodinium infection. BMC Genomics. 2019;20(1):1-15.

61. Sunagawa S, Wilson EC, Thaler M, Smith ML, Caruso C, Pringle JR, et al. Generation and analysis of transcriptomic resources for a model system on the rise: The sea anemone Aiptasia pallida and its dinoflagellate endosymbiont. BMC Genomics. 2009;10(10):258.

62. Boller T, Felix G. A Renaissance of Elicitors: Perception of Microbe-Associated Molecular Patterns and Danger Signals by Pattern-Recognition Receptors. Annu Rev Plant Biol. 2009;60:379-406. 
63. Dunn S. Immunorecognition and immunoreceptors in the Cnidaria. ISJ. 2009;6:714.

64. Song X, Jin P, Qin S, Chen L, Ma F. The Evolution and Origin of Animal TollLike Receptor Signaling Pathway Revealed by Network-Level Molecular Evolutionary Analyses. PLoS One. 2012;7(12).

65. Tassia MG, Whelan N V, Halanych KM. Toll-like receptor pathway evolution in deuterostomes. PNAS. 2017;114(27):7055-60.

66. Halanych KM, Kocot KM. Repurposed Transcriptomic Data Facilitate Discovery of Innate Immunity Toll-Like Receptor (TLR) Genes. Biol Bull. 2014;227:201-9.

67. Reitzel AM, Sullivan JC, Traylor-Knowles N, Finnerty JR. Genomic survey of candidate stress-response genes in the estuarine anemone Nematostella vectensis. Biol Bull. 2008;214(3):233-54.

68. Rauta PR, Samanta M, Dash HR, Nayak B, Das S. Toll-like receptors (TLRs) in aquatic animals: Signaling pathways, expressions and immune responses. Immunol Lett. 2014;158(1-2):14-24.

69. Morgan MJ, Liu Z. Crosstalk of reactive oxygen species and NF- $\kappa B$ signaling. Nat Publ Gr. 2010;21(1):103-15.

70. Wang M, Wang L, Jia Z, Wang X, Yi Q, Zhao L, et al. The versatile functions of LRR-only proteins in mollusk Chlamys farreri. Dev Comp Immunol. 2017 Dec;77:188-99.

71. Bosch TCG, Augustin R, Anton-Erxleben F, Fraune S, Hemmrich G, Zill H, et al. Uncovering the evolutionary history of innate immunity: The simple metazoan Hydra uses epithelial cells for host defence. Dev Comp Immunol. 2009;33:55969.

72. Franzenburg S, Fraune S, Künzel S, Baines JF, Domazet-Lo So T, Bosch TCG. MyD88-deficient Hydra reveal an ancient function of TLR signaling in sensing bacterial colonizers. PNAS. 2012;109(47):19374-9.

73. Bosch TCG. Cnidarian-Microbe Interactions and the Origin of Innate Immunity in Metazoans. Annu Rev Microbiol. 2013;67:499-518.

74. Gauthier MEA, Pasquier L Du, Degnan BM. The genome of the sponge Amphimedon queenslandica provides new perspectives into the origin of Toll-like and interleukin 1 receptor pathways. Evol Dev. 2010;12(5):519-33. 
75. Brown T, Bourne D, Rodriguez-Lanetty M. Transcriptional Activation of $\mathrm{c} 3$ and hsp70 as Part of the Immune Response of Acropora millepora to Bacterial Challenges. PLoS One. 2013;

76. Brown T, Rodriguez-Lanetty M. Defending against pathogens - immunological priming and its molecular basis in a sea anemone, cnidarian. Sci Rep. 2015;5:17425.

77. Croset V, Rytz R, Cummins SF, Budd A, Brawand D, Kaessmann H, et al. Ancient protostome origin of chemosensory ionotropic glutamate receptors and the evolution of insect taste and olfaction. PLoS Genet. 2010;6(8):e1001064.

78. Jékely G. Origin and early evolution of neural circuits for the control of ciliary locomotion. Proc R Soc B. 2011;278:914-22.

79. Derby CD, Steullet P. Why Do Animals Have So Many Receptors? The Role of Multiple Chemosensors in Animal Perception. Biol Bull. 2001;200:211-5.

80. Benton R. Multigene Family Evolution: Perspectives from Insect Chemoreceptors. Trends Ecol Evol. 2015;30(10):590-600.

81. Bigge CF. lonotropic glutamate receptors. Curr Opin Chem Biol. 1999;3:441-7.

82. Rytz R, Croset V, Benton R. Ionotropic Receptors (IRs): Chemosensory ionotropic glutamate receptors in Drosophila and beyond. Insect Biochem Mol Biol. 2013;43:888-97.

83. Grasso LC, Hayward DC, Trueman JWH, Hardie KM, Janssens PA, Ball EE. The Evolution of Nuclear Receptors : Evidence from the Coral Acropora. Mol Phylogenet Evol. 2001;21(1):93-102.

84. Goldstone J V. Environmental sensing and response genes in cnidaria: The chemical defensome in the sea anemone Nematostella vectensis. Cell Biol Toxicol. 2008;

85. Pierobon P. Coordinated modulation of cellular signaling through ligand-gated ion channels in Hydra vulgaris (Cnidaria, Hydrozoa). Int J Dev Biol. 2012;56(68):551-65.

86. Moroz LL, Kocot KM, Citarella MR, Dosung S, Norekian TP, Povolotskaya IS, et al. The ctenophore genome and the evolutionary origins of neural systems. Nature. 2014;510. 
87. Moroz LL, Kohn AB. Unbiased View of Synaptic and Neuronal Gene Complement in Ctenophores: Are There Pan-neuronal and Pan-synaptic Genes across Metazoa? Integr Comp Biol. 2015;55(6):1028-49.

88. Abuin L, Bargeton B, Ulbrich MH, Isacoff EY, Kellenberger S, Benton R. Functional Architecture of Olfactory Ionotropic Glutamate Receptors. Neuron. 2011;69:44-60.

89. Hart AC, Chao MY. From odors to behaviors in Caenorhabditis elegans. The Neurobiology of Olfaction. Front Neurosci. 2010;

90. Liebeskind BJ, Hofmann HA, Hillis DM, Zakon HH. Evolution of Animal Neural Systems. Annu Rev Ecol Evol Syst. 2017;48:377-98.

91. Benton R, Vannice KS, Gomez-Diaz C, Vosshall LB. Variant Ionotropic Glutamate Receptors as Chemosensory Receptors in Drosophila. Cell. 2009;136(149-162).

92. Forde BG. Glutamate signalling in roots. J Exp Bot. 2014;65(3):779-87.

93. Davenport R. Glutamate Receptors in Plants. Ann Bot. 2002;90:549-57.

94. Tapken D, Anschütz U, Liu L, Huelsken T, Seebohm G, Becker D, et al. A Plant Homolog of Animal Glutamate Receptors Is an Ion Channel Gated by Multiple Hydrophobic Amino Acids. Sci Signal. 2013;6(279):ra47.

95. Kwaaitaal M, Huisman R, Maintz J, Adler AR, Panstruga R. Ionotropic glutamate receptor (iGluR)-like channels mediate MAMP-induced calcium influx in Arabidopsis thaliana. Biochem J. 2011;440:355-65.

96. Kwaaitaal M, Maintz J, Cavdar M, Panstruga R. On the ligand binding profile and desensitization of plant ionotropic glutamate receptor (iGluR)-like channels functioning in MAMP-triggered Ca2+ influx. Plant Signal Behav. 2012;7(11):1373-7.

97. Leys SP. Elements of a 'nervous system' in sponges. J Exp Biol. 2015;218:58191.

98. Riesgo A, Farrar N, Windsor PJ, Giribet G, Leys SP, Mcdonald JH. The Analysis of Eight Transcriptomes from All Poriferan Classes Reveals Surprising Genetic Complexity in Sponges. Mol Biol Evol. 2014;31(5):1102-20.

99. Francis WR, Eitel M, Vargas S, Adamski M, Haddock SHD, Krebs S, et al. The genome of the contractile demosponge Tethya wilhelma and the evolution of metazoan neural signalling pathways. bioRxiv. 2017;preprint. 
100. Alberstein R, Grey R, Zimmet A, Simmons DK, Mayer ML. Glycine activated ion channel subunits encoded by ctenophore glutamate receptor genes. Proc Natl Acad Sci. 2015;10:E6048-57.

101. Moroz LL. Convergent evolution of neural systems in ctenophores. J Exp Biol. 2015;218:598-611.

102. Wang T-T, Si F-L, He Z-B, Chen B. Genome-wide identification, characterization and classification of ionotropic glutamate receptor genes (iGluRs) in the malaria vector Anopheles sinensis (Diptera: Culicidae). Parasit Vectors. 2018;11(34).

103. Greer JB, Khuri S, Fieber LA. Phylogenetic analysis of ionotropic L- glutamate receptor genes in the Bilateria, with special notes on Aplysia californica. BMC Evol Biol. 2017;17(11).

104. Willard SS, Koochekpour S. Glutamate, Glutamate Receptors, and Downstream Signaling Pathways. Int J Biol Sci. 2013;9:949-59.

105. Nakanishi N, Shneider NA, Axel R. A Family of Glutamate Receptor Genes: Evidence for the Formation of Heteromultimeric Receptors with Distinct Channel Properties. Neuron. 1990;5:569-81.

106. Lewerenz J, Maher P. Chronic Glutamate Toxicity in Neurodegenerative Diseases_-What is the Evidence? Front Neurosci. 2015 Dec 16;9:469.

107. Glezer I, Zekki H, Scavone C, Rivest S. Modulation of the innate immune response by NMDA receptors has neuropathological consequences. J Neurosci. 2003;23(35):11094-103.

108. Ramos-Vicente D, Ji J, Gratacòs-Batlle E, Gou G, Reig-Viader R, Luís J, et al. Metazoan evolution of glutamate receptors reveals unreported phylogenetic groups and divergent lineage-specific events. Elife. 2018;7:e35774.

109. Putnam NH, Srivastava M, Hellsten U, Dirks B, Chapman H, Salamov A, et al. Sea Anemone Genome Reveals Ancestral Eumetazoan Gene Repertoire and Genomic Organization. Science (80- ). 2007;317(5834):86-94.

110. Ryan JF, Pang K, Schnitzler CE, Nguyen A-D, Moreland RT, Simmons DK, et al. The genome of the ctenophore Mnemiopsis leidyi and its implications for cell type evolution. Science (80- ). 2013;342(6164):1242592.

111. Watanabe H, Fujisawa T, Holstein TW. Cnidarians and the evolutionary origin of the nervous system. Dev Growth Differ. 2009;51:167-83. 
112. Westfall JA, Elliott CF. Ultrastructure of the Tentacle Nerve Plexus and Putative Neural Pathways in Sea Anemones. Biology (Basel). 2002;121(3):202-11.

113. Norekian TP, Moroz LL. Neuromuscular organization of the Ctenophore Pleurobrachia bachei. J Comp Neurol. 2019;527(2):406-36.

114. Westfall JA, Landers DD, McCallum JD. Ultrastructure of neuro-spirocyte synapses in the sea anemone Aiptasia pallida (Cnidaria, Anthozoa, Zoantharia). J Morphol. 1999 Aug 1;241(2):165-73.

115. Westfall JA. Neural pathways and innervation of cnidocytes in tentacles of sea anemones. Hydrobiologia. 2004;530/531:117-21.

116. Strömberg SM, Östman C. The cnidome and internal morphology of Lophelia pertusa (Linnaeus, 1758) (Cnidaria, Anthozoa). Acta Zool. 2017;98(2):191-213.

117. Zbek S, Balasubramanian PG, Holstein TW. Cnidocyst structure and the biomechanics of discharge. Toxicon. 2009;54:1038-45.

118. Westfall JA, McCallum JD, Carlin RW. Neuroglandular synapses in the pharynx of the sea anemone Aiptasia pallida (Cnidaria, Anthozoa). J Morphol. $2001 \mathrm{Feb}$ $1 ; 247(2): 134-41$.

119. Wudick MM, Michard E, Oliveira Nunes C, Feijó JA, Nunes CO, Feijó JA. Comparing plant and animal glutamate receptors: common traits but different fates? J Exp Bot. 2018;

120. Krishnan A, Schiöth HB. The role of G protein-coupled receptors in the early evolution of neurotransmission and the nervous system. J Exp Biol. 2015;218:56271.

121. Brown T. Phenomenological and Molecular Basis of the Cnidarian Immune System. Florida International University; 2017.

122. Sorek M, Schnytzer Y, Waldman Ben-Asher H, Chalifa Caspi V, Chen C-S, Miller DJ, et al. Setting the pace: host rhythmic behaviour and gene expression patterns in the facultatively symbiotic cnidarian Aiptasia are determined largely by Symbiodinium. Microbiome. 2018;6(83).

123. Bosch TCG. Rethinking the role of immunity: lessons from Hydra. Trends Immunol. 2014;35(10):495-502. 


\section{CHAPTER II. CNIDARIAN-SPECIFIC EXPANSION OF IONOTROPIC \\ GLUTAMATE RECEPTORS PROMOTE NEOFUNCTIONALIZATION WITH IMPLICATIONS FOR CHEMOSENSORY IMMUNITY}

\subsection{Abstract}

Ionotropic glutamate receptors (iGluRs) are transmembrane proteins involved in a variety of biological processes from chemical-mediated neuron communication within vertebrate brains to chemosensation within insect antennae. Recently identified iGluRs in cnidarians have been postulated to function as danger-sensing and/or pathogen receptors in immune defense. To address the hypothesis, we explored the extent of phylogenetic diversity of iGluR proteins in available cnidarian sequenced genomes with particular interest on the cnidarian-dinoflagellate model system, the sea anemone Exaiptasia pallida. Furthermore, functional gene expression of multiple iGluR genes was measured from E. pallida in response to bacterial pathogen exposure. Our findings revealed a greater evolutionary expansion of cnidarian iGluR lineages than recently reported, and the discovery of a new cnidaria-specific class. Some cnidarian iGluRs maintain conserved domains while others have a reduced domain composition indicative of functional divergence in the cnidarian iGluR protein family. Gene expression differentiation of iGluRs suggests select genes respond transcriptionally to bacterial challenge, supporting the hypothesis that cnidarian iGluRs respond to pathogen signals. Altogether, cnidarian iGluRs expansions indicate cnidarian-specific neofunctionalization towards functions as chemosensory receptors, including immune chemosensing specific to bacterial infectious agents. 
2.2 Keywords: Ionotropic Glutamate Receptor; iGluR; chemosensory; Cnidarian; Exaiptasia pallida; coral immunity.

\subsection{Introduction}

Organisms have an ecological interface provided through immunity; the designated system composed of essential barriers and recognition proteins, which works defensively against harmful, infectious agents. Even the minutest microscopic single-living prokaryotes i.e., bacteria, possess innate immune mechanisms to defend against viral infections $(1,2)$. In recent years, interest to understand how invertebrate immune defense systems evolved has grown (3-7), but the focus remains heavily biased towards vertebrate models with direct applications to human health. Unlike vertebrates, earlydiverged metazoans lack of an adaptive of immune system and rely upon innate functions, coded within their genomes, to identify and respond to microbial and/or chemical cues to recognize pathogenic agents (6-10). The dependence on an innate immune system for defense against the countless microorganisms encountered during a lifespan among invertebrates poses one of the greatest conundrums in comparative immunology $(6,11)$.

A widely used approach to understand invertebrate innate immunity is identifying homologous genes of well-characterized genes in the vertebrate immune system. Although the transfer of information from vertebrates to invertebrates derived from homology is informative for understanding the functionality of orthologous genes, it is 
less informative in gene families that have undergone several rounds of lineage-specific gene duplication and limited in its application of immune mechanisms unique to earlydiverged metazoans (9). While homologous genes implicated in innate immunity has been identified in invertebrates $(3,4,6,7,12-14)$, the detection of homologs in established functional pathways alone, without experimental evidence, does not lead to full comprehension of gene function. A complementary approach to elucidate immune functionality is by identifying expression changes of genes during pathogenic or antagonistic elicitor of immune defense. Using this approach, a number of genes involved in the defense response of the sea anemone, Exaiptasia pallida (Agassiz in Verrill, 1864), to the coral-pathogenic bacterium Vibrio coralliilyticus were identified (15). One of the discovered genes with increased expression encoded a chemosensory ionotropic glutamate receptor (iGluR) concurrent to improved resistance against repetitive bacterial challenges (15). The novel discovery of iGluR involvement in immune response of cnidarians aligns with similar findings in plants $(16,17)$.

One important function of the immune system of marine organisms is to recognize potentially infectious agents, and for that chemosensory machinery might play an essential role. Ionotropic glutamate receptors (iGluRs) represent one particular "flavor" of chemosensory receptor proteins, which are embedded within the cell membrane to send external signals into the cell and elicit a physiological reaction or behavior $(18,19)$. Chemosensing-associated behaviors include prey captures, predator evasion and other functions essential to survivorship described in early-divergent metazoans (20-22). Glutamate or a specific ligand binds to the ligand-binding region of 
an iGluR and activates the opening of its ion channel to allow transport of calcium ions into the cytoplasm of the cell and further system responses $(19,23)$. The iGluR protein family is known for neuronal communication in sensory responses to environmental factors $(21,24)$; without a central nervous system (CNS), cnidarians use a nerve net, or diffused nervous system of sensory and ganglionic neurons spaced between epithelial cells to interpret iGluR signals (25-27). The nerve net present in anthozoans i.e., nonsymbiotic anemone Nematostella vectensis, is an arrangement of linear nerve cords that send directional conduction to trigger local muscle contractions (28-30). The iGluRs identified in non-symbiotic Hydra vulgaris modulate nematocyst discharge as a response to prey or self-defense (29), supporting the hypothesis that iGluRs act consequently to triggered defense signals. Alternatively, if acting similarly to chemosensation in plants, cnidarian iGluRs could function as a component of the danger recognition response to tissue damage accrued by the presence of a pathogen rather than an antigen response to modulate chemical stresses and wound healing (13,31-33).

To characterize gene diversity and infer evolution of iGluR proteins in cnidarians, we explored homologs in available sequenced genomes including actinarians and scleractinian corals with particular interest on the cnidarian-dinoflagellate model system, the sea anemone Exaiptasia pallida (34). Moreover, we investigated through gene expression assays the involvement of E. pallida iGluRs in the immune response to infectious bacterial challenges. This study aids in interpreting the evolution of cnidarian iGluRs and putative functional role in bacterial chemosensing. 


\subsection{Materials and methods}

\subsubsection{General metazoan dataset and phylogeny: Ionotropic GluR sequences were}

extracted from sequence databases to carry out phylogenetic analyses with the objective of inferring the evolution of iGluRs across model metazoans with particular interest on cnidarians. Based on isoform 1 from the previously identified cnidarian iGluR gene for $E$. pallida (KXJ14767), additional iGluR homologs were identified from the Reference Protein database for E. pallida (34) using default NCBI BLAST with an e-value cutoff of $1 \times 10^{-10}$. In the case of multiple protein isoforms from the same gene, the longest isoform was chosen. The sequences from E. pallida were numbered in order of blastp hits (i.e., GluR (query), GluR3, GluR4, GluRn, etc.). The two isoforms from GluR (KXJ4767) were labeled as gluR.1 (XP_020899710) and gluR.2 (XP_020899711). The resulting E. pallida protein sequences and the complete canonical proteomes from Uniprot containing one primary protein isoform from each gene from one Chlorophyta, and 15 metazoans species were combined into a local blast database. Sequences were labeled with species genus initials and accession number (Table 2.S1, S2). A local blastp (BLAST 2.2.30+) was run against the database using GluR.1 as a query with a cutoff e-value of $1 \times 10^{-10}$. Sequences shorter than 200aa in length and sequences with less than $20 \%$ identity were excluded while remaining sequences were aligned using the iterative refinement method (L-INS-i), incorporating local pairwise alignment information for accuracy in Mafft v7.221 in Terminal (35). The best model of evolution ( $\mathrm{LG}+\mathrm{G} 6+\mathrm{I}+\mathrm{F})$ was determined with Smart Model Selection from PhyML (36). BlackBox v8.2.4 RAxML performed Maximum Likelihood analysis using the best model of evolution and defaults within 
CIPRES portal (37). The resulting phylogeny of iGluRs was rooted with the two sequences from outgroup C. reinhardtii and is referred to as the general metazoan iGluR phylogeny.

2.4.2 Cnidarian-specific dataset and phylogeny: Using the general metazoan phylogeny, sequences from select clades were extracted and built upon with additional cnidarian sequences to improve the resolution of the evolution of iGluR in cnidarians. Sequences from nine scleractinian coral species from the Reef Genome project and from two non-cnidarians Oscarella carmela (Porifera) and Mnemiopsis leidyi (Ctenophora) species were used to build a second database (Table 2.S1 and 2.S2). Through a local blastp search against this database with gluR.1 as the query, sequences with an e-value of $1 \times 10^{-10}$ that were at least 200aa long, and had at least $20 \%$ identity were added to the extracted dataset from the general metazoan phylogeny. The resulting set of sequences was aligned using the iterative refinement method (L-INS-i) in Mafft v7.221 (35). The best model of evolution $(\mathrm{LG}+\mathrm{G} 6+\mathrm{I}+\mathrm{F})$ was determined with Smart Model Selection from PhyML (38). BlackBox v8.2.4 RAxML performed Maximum Likelihood analysis using the best model of evolution and defaults within CIPRES portal (37). The resulting phylogeny of iGluRs was rooted with the two sequences from outgroup C. reinhardtii and is referred to as the cnidarian iGluR phylogeny.

\subsubsection{Modeling the molecular structure of GluRs for functional insights: Protein} domains across all GluRs were identified within Pfam (39). Homology modeling of E. pallida iGluRs was performed to compare structure and putative molecular function 
across the phylogeny. SWISS-MODEL from ExPASy was run with defaults to identify templates for subunit 3D models (40-46). Template searches were performed through BLAST and HHBlits against the SWISS-MODEL template library (SMTL) $(40,41,43-$ 49). Models were built from target-template alignment using ProMod3 defaults. Templates with highest quality predicted from target-template alignment features were selected using GMQE, while global and per-residue qualities of the models were evaluated through the QMEAN scoring function in SWISS-MODEL (50). The PyMol v2.2 program was used for viewing 3D model structures (51).

\subsubsection{Investigation of Exaiptasia iGluRs transcriptional expression under bacterial}

challenge: To investigate the putative functional involvement of Exaiptasia iGluRs in immune response, changes of transcriptional expression was assessed in anemones exposed to established bacterial pathogens. For this, we performed a similar experiment conducted within our laboratory designed to study immunological priming from which we discovered the first iGluR implicated immune defense $(15,52)$. The bacterial challenges were performed with known coral pathogens $V$. coralliilyticus and Serratia marcescens $(52,53)$ in sub-lethal conditions in order to trigger an immune defense response without compromising survivorship.

2.4.5 Animal maintenance: Clonal symbiotic E. pallida anemones (CC7) were used in the experiment and maintained in artificial seawater at approximately $27^{\circ} \mathrm{C}$. Populations were kept on a day/night cycle of 12 hours light: 12 hours dark with 30 to $60 \mu \mathrm{mol}$ photons $\mathrm{m}^{-2} \mathrm{~s}^{-1}$ of light intensity and fed freshly hatched brine shrimp Artemia nauplii 
twice a week. Exaiptasia pallida anemones were approximately $3 \mathrm{~mm}$ in diameter and $8 \mathrm{~mm}$ in length.

\subsubsection{Culturing bacteria (Vibrio coralliilyticus and Serratia marcescens) and} experimental design of bacterial challenges: The strains $V$. coralliilyticus BAA 450

(ATCC) and $S$. marcescens PDL 100 were used for challenge experiments. Bacteria were streaked from glycerol stocks stored at $-4{ }^{\circ} \mathrm{C}$ and grown overnight on Marine Agar (Difco, USA) at $30^{\circ} \mathrm{C}$. The following day, a single colony was picked with an inoculating loop and grown to logarithmic phase overnight at $30{ }^{\circ} \mathrm{C}$ while shaking at 100 rpm in Marine Broth-2216 (Difco, USA). Cultures were centrifuged at 10,000 xg for five minutes, washed and re-suspended in sterile seawater before starting the infection trials. Bacterial cells were prepared to the designated final concentration of $10^{8} \mathrm{CFU} \mathrm{mL}^{-1}$ calculated from growth curves generated by absorbance (optical density readings at 600 $\mathrm{nm}$ ) plotted against known $\mathrm{CFU} \mathrm{mL} \mathrm{m}^{-1}$. Infection experiments were conducted at $30{ }^{\circ} \mathrm{C}$ and at a final concentration of $10^{8} \mathrm{CFU} \mathrm{mL}^{-1}$ of $V$. coralliilyticus or $S$. marcescens following the protocol set forth by Brown and Rodriguez-Lanetty (15). Individual $E$. pallida anemones were acclimated to the experimental temperature $\left(30^{\circ} \mathrm{C}\right)$ for $24 \mathrm{hr}$ prior to experiment and placed into a single well of a twelve-well tissue culture plate containing artificial seawater. Anemones were sub-lethally challenged for $72 \mathrm{hr}$ with $V$. coralliilyticus or $48 \mathrm{hr}$ with $S$. marcescens as described in Brown \& Rodriguez-Lanetty (15) and Brown (52) (Fig. 2.S2). Sub-lethal exposures allow animals to engage in immunological defense without causing mortality. After completion of challenge exposures, anemones were transferred to new 12-well tissue culture plates with clean 
seawater. Anemones were sampled at six time points $(0,24,48,72,96 \mathrm{hr}$, and $4 \mathrm{wk})$. Zero hours marks the time of inoculation. Control anemones were moved between 48 and $72 \mathrm{hr}$ to new 12-well plates to mirror the bacteria-challenged treatments transfer and kept at $30{ }^{\circ} \mathrm{C}$ for the entire experiment and not challenged with bacteria. Anemones were snap-frozen in liquid nitrogen and stored at $-80^{\circ} \mathrm{C}$ until processed for RNA extraction.

2.4.7 RNA extraction and cDNA synthesis: We used the RNeasy mini kit (Qiagen, Valencia, CA) to extract RNA. The samples were applied to spin columns from the RNeasy kit and washes performed following the manufacturer's protocol using the oncolumn DNase treatment (Qiagen, Valencia, CA) and two additional 80\% ethanol washes. RNA was eluted in a final volume of $30 \mu \mathrm{L}$ of nuclease-free water. Quantification of extracted RNA was conducted using a NanoDrop 2000 (ThermoFisher, Carlsbad, CA) and RNA integrity assessed visually on a $1 \%$ TAE bleach gel (54). Samples with a spectrophotometric $260 / 230$ ratio of less than 1.5 underwent additional cleanup using $10 \% 3 \mathrm{M}$ sodium acetate ( $\mathrm{pH}$ 5.2), RNA-grade glycogen at a final concentration of $0.05-$ $1.0 \mu \mathrm{g} / \mathrm{mL}$, and 2.5 volumes $100 \%$ ethanol to the sample. Following precipitation overnight at $-80{ }^{\circ} \mathrm{C}$, samples were centrifuged for $20 \mathrm{~min}$ at $9500 \mathrm{rpm}\left(4^{\circ} \mathrm{C}\right)$ to pellet RNA. The pellet was washed with $250 \mu \mathrm{L} 70 \%$ ethanol, centrifuged for $5 \mathrm{~min}$ at the same conditions listed above, and dried before re-suspending in $20 \mu \mathrm{L}$ nuclease-free water. Complementary DNA synthesis was performed with Superscript III first strand synthesis system (Life Technologies, Eugene, OR) using $120 \mathrm{ng}$ of RNA and Oligo dT primer. 
2.4.8 Quantitative RealTime-PCR assays: Primers with a length of 18 to 25 base pairs and an optimal melting temperature $(\mathrm{Tm})$ of $60^{\circ} \mathrm{C}$ were designed using PrimerQuest (IDT, Coralville, IA). Through comparing to the Exaiptasia genome (34), primer pairs were designed across introns to prevent genomic DNA amplification. Nine working primers were utilized for qPCR amplifications targeting six of the identified E. pallida iGluRs (GluR) and three reference genes (Table S3). Primer concentrations were optimized by comparing qPCR reads using $0.5 \mathrm{nM}$ and $0.25 \mathrm{nM}$ of primer in order to minimize primer dimer formation and maximize output read. For qPCR analysis, a master mix of SsoAdvanced Universal SYBR Green Supermix (BioRad, Hercules, CA), 0.34 $\mathrm{nM}$ of forward and reverse primers and nuclease-free water was added in $19.5 \mu \mathrm{L}$ aliquots to each well with $0.5 \mu \mathrm{L}$ of sample template. Plates for each gene were run on Bio-Rad CFX Connect systems at a thermocycle of initial polymerase activation of $30 \mathrm{~s}$ at $98^{\circ} \mathrm{C}$, followed by 38 cycles of $15 \mathrm{~s}$ denaturation at $95^{\circ} \mathrm{C}$, then annealing for $30 \mathrm{~s}$ at $54^{\circ} \mathrm{C}$, followed by extension for $30 \mathrm{~s}$ at $72{ }^{\circ} \mathrm{C}$ with a plate read, and final Melt-Curve analysis. Plates had an Inter-Run Calibrator to control for variation of reads between runs. Negative controls for No Reverse Transcriptase were run for all samples with primers to confirm no genomic DNA contamination or bias. Raw output was corrected for variations between each run using an inter run/plate control within the BioRad program. PCR amplification efficiency was corrected through LinReg v.2016.1 (55). Gene expression was normalized using the geometric means of three reference genes, ribosomal proteins L10 and L12, and poly (A)-binding protein (56,57). Fold change of relative quantities was found by subtracting control average of each time point from treatment replicates to measure difference in expression caused by the bacterial challenge. Statistical analysis to 
compare bacterial challenges effect on fold change expression across time was completed by linear model 2-way ANOVA using Tukey HSD post hoc multiple comparisons of means with a 95\% family-wise confidence level R v3.5.0 (58). Data normality and homoscedasticity were tested using Shapiro-Wilks test (59) or Levene-Bartlett's test, respectively. In cases where ANOVA assumptions were not fulfilled, data was transformed using Tukey's Ladder of Power transformation and analyzed using White's adjusted ANOVA in R v3.5.0 using car, MASS, and rcompanion, glue, and "userfriendlyscience" packages (60-63).

\subsection{Results}

2.5.1 Identification of iGluR homologs: One hundred sequences from Cnidaria met the criteria to be identified as iGluR homologs in our Reef Genome proteome database. An additional 265 homologs were identified in our assembled Uniprot reference proteome database (Table 2.S1, 2.S2).

\subsubsection{Phylogenetic inferences of cnidarian iGluRs to representative iGluR groups:}

To explore the evolutionary history of iGluRs in Cnidaria, two phylogenies were built. The first phylogeny covered a selection of metazoan taxa using Chlamydomonas reinhardtii as the outgroup (Fig. 2.S1). For all taxa, except E. pallida, the Uniprot reference proteomes were used to compile an assessment of the presence or absence of iGluRs (Table 2.S1). For E. pallida, iGluRs were identified from the Reef Genomics protein database, resulting in 22 paralogs (Table 2.S1). The reference proteome tree 
revealed the presence of eight clades. Clades 1 and 2 diverged earlier than the rest of the clades. Clade 1 was represented by orthologs of NMDA-specific GluN1 (sensu Collingride et al. 65) found in most metazoans (Fig. 2.S1). Clade 2 was composed of NMDA-GluN2 and NMDA-GluN3 orthologs, which were well represented across Metazoa with major lineage-specific expansion in Cnidaria and to a lesser extent in mollusks and tardigrades (Fig. 2.S1). Clade 3 was conformed by non-NMDA iGluR orthologs from Placozoa (Trichoplax adhaerens) and Cnidaria, each with their own lineage-specific expansions (Fig. 2.S1). Clade 4 held another cnidarian-specific expansion of non-NMDA iGluRs with strong node support (Fig. 2.S1). Clade 5 was represented by ionotropic receptors (IR) that are missing from chordates, but highly duplicated within mollusks. Clades 6, 7, and 8 demonstrate broad lineage-specific expansions (Fig. 2.S1). Notably, clades 5 to 8 are devoid of cnidarian sequences.

To improve resolution of the cnidarian part of the tree, sequences from clades 1-4 were extracted and combined with an expanded set of sequences from Cnidaria, Ctenophora, and Porifera (Tables 2.S1, 2.S2). The resulting tree overall confirmed the clades in the previous larger tree and further placed ctenophore sequences in one major clade that shows a high level of lineage specific expansion (Fig. 2.1; CX). Porifera fell within C3, together with $T$. adhaerens and Cnidaria, suggesting that this clade was present in early Metazoa. As in the previous tree, $\mathrm{C} 4$ held only cnidarian sequences. Although the sequence set from Acropora millepora and other corals from the Reef Genomics project is incomplete, their placements within the phylogeny strongly suggest they follow the pattern of E. pallida, N. vectensis, and S. pistillata (Fig. 2.1). 


\subsubsection{Molecular modeling of Exaiptasia pallida GluRs with functional insights: Three}

Pfam domains (39) are characteristic of iGluRs, an amino (or N-) terminal domain (ATD), a ligand-specific binding domain (LBD), and a transmembrane domain (TMD) that is an ion channel. Conserved domains for iGluRs identified within Pfam (39) showed multiple E. pallida iGluRs contain all three conserved domains (Fig. 2.2). The ATD was identified as atrial natriuretic factor receptor family ligand binding region (PF010904/IPR001828) in GluN, GluA, GluK and IR8a and 25a, but it is missing within select GluR sequences (Fig. 2.2). The LBD often characterized as ligand channel glutamate binding domain (PF10613/IPR019594), contains two highly conserved regions, 2.S1 and 2.S2, which form a clamp for binding solutes or ligands (Fig. 2.2). The LBD includes the sequence motif SYTANLAAFL, conserved in all chordate iGluRs (65-67), but where threonine substitutes serine in GluRs. The LBD region is also identified as bacterial extracellular solute-binding protein family 3 (PF00497/IPR001638) that is closely associated to glutamate-binding domains (Fig. 2.2) (68). The TMD region identified as the ligand-gated ion channel domain (PF00060/IPR001320) is mostly conserved within GluRs (Fig. 2.2). In GluR22 (C1) and GluR6 (C3), the TMD correspond to a calmodulin-binding domain of NMDA receptor 1 subunit (PF10562/IPR018882) (Fig. 2.2). GluR19 (C2) has one domain that is not characterized within Pfam (Fig. 2.2). Of all GluRs, the sequences that include all domains are within $\mathrm{C} 2: 11,18,16,13,14$, within $\mathrm{C} 3: 7$, within $\mathrm{C} 4: .1, .2,3,9,5$, and 4, although some domains are incomplete. 
Homology models were built for two representative cnidarian iGluRs using the SWISS-MODEL server (40-46). The two representative proteins with all three typical iGluR domains were GluR11 (C2) and GluR.1 (C4) (Fig. 2.1 \& 2). The homology models were constructed based on two different templates, identified from the global GMQE values (normalized values of 0.58 and 0.56 ). For GluR11 (C2), the template was a structure of the activated GluA2 from a chimeric sequence Rattus norvegicus and Mus musculus (PDB ID: 5WEO, chain A). The sequence identity for the target and template was $32 \%$ and $84 \%$ of the target was modeled (Fig. 2.3A). For GluR.1 (C4) the template was an apo structure representing the resting state of GluA2 as determined from $R$. norvegicus (PDB ID: 4U2P, chain B). Sequence identity for the target and template was $31 \%$ and $86 \%$ of the target was modeled (Fig. 2.3B). Both proteins were modeled as homotetramers to represent the functional iGluR unit (Fig. 2.3). The local QMEANs of the models were -4.93 and -4.87 , respectively for GluR11 (C2) and GluR.1 (C4) (Fig. 2.3). The QMEAN values indicate low quality models for globular protein models, but can be expected for transmembrane receptors $(45,50)$.

\subsubsection{Transcriptional analysis of iGluR gene expression following bacterial}

challenge: To test whether the transcriptional expression of discovered E. pallida iGluR proteins responded to infectious agents, gene expression was measured through qPCR analyses over six time points $(0,24,48,72,96 \mathrm{hr}$ and $4 \mathrm{wk})$ following bacterial exposure (Fig. 2.S2; Fig. 2.4). Out of designed iGluRs primers, six provided successful amplifications. Primers targeted gluR.1 (C4), gluR.2 (C4), gluR4 (C4), gluR8 (C4), gluR21 (C2), and gluR23 (C3) (Table S3). Designed primers targeting the sole C1 
sequence (gluR22) did not provide successful amplification; therefore, no transcriptional data was produced for C1 Exaiptasia ortholog. Expression of genes gluR23 (C3) and gluR21 (C2) showed significant differences as a function of bacterial challenge (White's adjusted ANOVA, $p=0.0003$ and $p=2.28 \mathrm{e}-5$, respectively; Fig. 2.4C-D; Table 2.S4). Exaiptasia pallida gluR21 (C2) transcription regulation was affect by the interaction of time and treatment (2-way ANOVA Tukey HSD, p=0.0190). Exaiptasia pallida gluR.1 (C4) and gluR.2 (C4) (isoforms of GluR differing by 24 sequential base pairs) also showed transcriptional responses to bacterial challenge (2-way ANOVA, $p=0.0414$ and $p=0.0016$, respectively; Table 2.S4), however, patterns differed over time. Serratia marcescens, significantly affect gluR.1 expression trends, with up-regulation at $96 \mathrm{hr}$ (2way ANOVA Tukey HSD, $\mathrm{p}=0.0319, \mathrm{p}=0.0299$; Table 2.S5). Up-regulation of gluR.1 (C4) under challenge of $V$. coralliilyticus appeared at $96 \mathrm{hr}$ and $4 \mathrm{wk}$, but was not measurably significant (2-way ANOVA, p=0.401; Fig. 2.4A; Table 2.S5). Exaiptasia pallida gluR.2 (C4) showed a similar trend of expression over time in both bacterial treatments. Expression appeared to increase at $48 \mathrm{hr}$ under the challenge of $S$. marcescens and transcriptional expression remained high during the subsequent times points (2-way ANOVA Tukey HSD, p=0.00112; Fig, 4B; Table 2.S5). Vibrio coralliilyticus had a borderline significant affect on expression regulation of gluR.2 (2-way ANOVA Tukey HSD; p=0.0746; Fig. 2.4B; Table 2.S5). Exaiptasia pallida gluR4 (C4) and gluR8 (C4) were not differentially expressed. 


\subsection{Discussion}

We have performed to date the most comprehensive phylogenetic analysis of cnidarian ionotropic glutamate receptors that has permitted us to develop a model for their evolution within metazoan. Our study also revealed functional differences based on protein domains in silico and the involvement of certain iGluRs groups in the recognition of biological danger in vivo, as part of the defense response of Exaiptasia pallida.

2.6.1 Evolutionary expansions of iGluRs in Cnidaria: Our findings revealed a greater expansion of cnidarian iGluRs than recent metazoan iGluR phylogenies reported $(21,69)$. The 22 Exaiptasia pallida iGluR genes are comparable in number to other Cnidaria and greater than most other metazoan species in the study (Table 2.S1). Only Lottia gigantea and Crassotrea gigas have more iGluR homologs, 45 and 31 genes, respectively (Table 2.S1). Our phylogenies confirm lineage-specific iGluR gene expansions across metazoan $(21,23,67,69-71)$ and reveal a distinctive pattern of iGluR expansion within the recently sequenced E. pallida genome (34) and other Cnidaria that resolved in four phylogenetic clades (Fig. 2.1 and 2.S1). We interpret our findings in the context of a recent phylogenetic hypothesis of the evolution of iGluR in metazoans, in which four subfamilies (Lamda, NMDA, Epsilon, and AKDF) and 10 classes were proposed (69). The GluN sequences from our phylogenetic trees were represented in clades $\mathrm{C} 1$ and $\mathrm{C} 2$, and both include cnidarian sequences along with other metazoan orthologs (Fig. 2.1 \& 2.S1). However, our tree topology is not congruent with the recent NMDA iGluR phylogeny (69). Ramos-Vicente and colleagues proposed four classes containing NMDA-specific 
(GluN) orthologs where two of them included cnidarian sequences (NMDA1: GluN1 and a cnidarian-specific NMDA class). Moreover, the cnidarian NMDA class was proposed to be an early-diverged sister clade of the other three NMDA-specific classes (GluN1, 2 and 3; sensu 70). On the contrary, in both our phylogenetic trees, GluN1 (C1) is the sister group of C2 that includes GluN2/3+cnidarian. Further, the branch lengths in $\mathrm{C} 1$ are shorter than in $\mathrm{C} 2$, suggesting that the $\mathrm{C} 1$ with $\mathrm{GluN}$ is closer to the ancestral state and the $\mathrm{C} 2$ with GluN2/3+ cnidarian more derived.

In regard to the other three resolved clades (C3, $C 4$ and $C X$; Fig. 2.1) represented by non-NMDA iGluRs, we noted that two Nematostella paralogs (NV_A7RPU4 and NV_A7T1G4) in C3 have been identified as belonging to the subfamily Epsilon (GluE1_Nve and GluE2_Nve, respectively; sensu Ramos-Vicente et al. (69) and two other Nematostella paralogs (NV_A7S4J5 and NV_A7S4J7) in C4 belong to the subfamily AKDF (GluAKDF1_Nve and GluAKDF_Nve2, respectively; sensu 70). This implicates that Cnidaria (in particular E. pallida) also possess a diverse representation of iGluRs in these two non-NMDA subfamilies. However, our findings suggest that C3 and $\mathrm{C} 4$ are phylogenetically more related to one another than to the monophyletic CX, represented by ctenophore iGluRs. Ramos-Vicente and colleagues suggested that ctenophores only have one type of iGluR from the subfamily Epsilon (69), which further implies that $\mathrm{C} 3$ and $\mathrm{C} 4$ also belong to subfamily Epsilon, the closest to subfamily NMDA. The two Nematostella sequences from the subfamily AKDF within C4 seem to be a misclassification by Ramos-Vicente et al. (69). This could be attributed to difference in sequence composition, such as insect IRs (C5) that allowed us to separate GluA- 
AMPA and GluK-Kainate orthologs (C8) and GluD-Delta (C6) from C3, C4 and CX. We proposed based on our phylogenetic analyses that the cnidarian specific Clade 4 is an expansion of non-NMDA iGluRs within subfamily Epsilon and should be considered a cnidarian class on its own within this subfamily (Fig. 5).

2.6.2 Neofunctionalization and subfuctionalization of cnidarian iGluRs: In cnidaria, numerous gene duplications are evident. The GluR co-orthologs within the four main clades may have similar functional characteristics but given the amount of lineagespecific gene duplications, the amount of amino acid substitutions as indicated by branch lengths, and altered domain compositions, this may not always be the case. The common scenario following gene duplication is that the resulting functional redundancy allows for relaxed selective pressures. Consequently, the different gene copies often show increased amino acid substitution rates and a more rapid exploration of sequence and function space. The majority of gene duplicates become pseudogenes (72), but those that are retained either do not change in function or go through a process of neo- and subfunctionalization $(73,74)$. For E. pallida, several sequences in our dataset lack all characteristic iGluR domains (Fig. 2.2), which is consistent with sub- and neofunctionalization. These sequences may be partial sequences in the database and fulllength in the actual genome, but they may also have functionally diverged by mechanisms beyond amino acid substitutions such as domain loss. Like co-receptor ionotropic receptors (IR) within insects, GluR22, 19, 17, 6, and 15 (Fig. 2.2) lack of ATD $(18,20,23)$. Loss of the ATD appears to have happened in GluRs in all four clades at different times, suggesting that this loss may benefit fitness. Amino acid substitutions of 
key residues in LBD or TMD can alter specificity to ligands or ions, respectively. The changes within regions of functional domains have potential to differentiate the functional roles via environmental ligands or signal pathways. The E. pallida sequences containing all representative iGluR domains may still function as archetypal iGluRs.

\subsubsection{Certain iGluR clades in $E$. pallida are functionally implicated in the response}

to bacterial exposure: The first indication that iGluRs had a role in cnidarian immune defense came from findings that revealed that immunological primed anemones showing resistance to repetitive bacterial infections had a significant increase of protein expression of GluR (C4) compared to naïve anemones that encountered the pathogen for the first time (15). In this study, we further examined the functional conservation in immune defense from the other iGluRs clades unveiled from our phylogenetic analyses by exploring whether bacteria exposure would elicit transcriptional expression changes across these GluRs. Through a functional exploration of six E. pallida iGluRs, the findings indicated that GluRs from other clades (apart from C4) also responded to bacterial challenges such was the case for iGluRs in clades $\mathrm{C} 2$ and $\mathrm{C} 3$. This implicates an immunological functionality of E. pallida iGluRs from both NMDA (at least C2) and Epsilon subfamilies ( $\mathrm{C} 3$ and $\mathrm{C} 4)$. However, the patterns of expression through the timecourse of this study differed among iGluRs and in some cases showed to be dependent on the bacterial pathogens. In general, the response of these iGluRs was more evident to bacterium Serratia marcescens than to Vibrio coralliilyticus, which might be associated with difference in infectivity dynamics between the two bacterial pathogens. 
The transcriptional increase of the isoforms gluR.1 and gluR.2 from C4 to a bacterial pathogen confirmed similar results for the same gene at proteomic level within the same experimental time-course (15). However, the other two tested iGluRs also from C4 (gluR4 and gluR8) did not change expression under the bacterial challenge indicating that not all Exaiptasia iGluRs in $\mathrm{C} 4$ are implicated in the response to bacterial infections, which suggests that some of them may be involved in other functional processes. While gluR8 may not be functional due to the lack of several iGluR functional domains (Fig. 2.2), gluR4, on the other hand, had all three characteristic iGluR domains and is expected to function as an iGluR. Moreover, it is worth noting that both these two gluR4 and gluR8 grouped in different sub-clades within $\mathrm{C} 4$ compared to the immunologically involved GluR (i.e. gluR.1/R.2) (Fig. 2.1), and this differential phylogenetic signature might relate to possible functional divergence within Epsilon (GluE) C4 (Fig. 2.5).

Initially, iGluRs were thought to be strictly involved with the nervous system (in synapses) but recent findings have revealed that even in mammals these glutamate receptors are implicated in the regulation of innate immune responses $(75,76)$ and in boosting adaptive immune T-cells (77). In plants, knockout mutants have shown that some iGluRs homologs, glutamate-like receptors (GLR), play important roles in defense response (32). Furthermore, a recent proposed model speculates that plant GLR ligands might in some cases function as danger-associated molecular patterns (DAMPs) and suggested that the sensing of different combinations of host and non-host derived components by GLRs during infections may be integrated to regulate alternative plant defense pathways (16). Our findings draw some parallels to what we have learned from 
plants; and provide new light on the functional property of iGluRs from early-diverged metazoans in immune defense and chemosensory associated to danger. Future studies using the Exaiptasia model system to determine the localization of expression of these receptors along with the identification of ligand specificity will provide further evidence to explain the mechanism in immune defense regulation and also to reveal other novel functions from other cnidarian iGluR clades.

\subsection{Acknowledgements}

The concept of the project was conceived by MRL. The experimental designed was planned by EGD, JSL, and MRL. EGD carried out all experiments and statistical analyses. Bioinformatic analyses were carried out by EGD with contributions from JSL. Manuscript was mostly written by EGD and edited by JSL and MRL. We also thanks to Dr. Matthew DeGennaro, Dr. Sheila A. Kitchen and Dr. Anthony Bellantuono for technical suggestions during parts of the project. 


\subsection{Tables and Figures}

Table 2.S1 Taxa included within analysis and abbreviations used along with the number of sequences per taxa included in the iGluR phylogenies for both cnidarian and general metazoan trees.

\begin{tabular}{|c|c|c|c|}
\hline Taxa & Abbreviation & Cnidarian & General Metazoan \\
\hline Exaiptasia pallida & EP & 22 & 22 \\
\hline Nematostella vectensis & NV & 19 & 19 \\
\hline Stylophora pistillata & SP & 26 & 26 \\
\hline Acropora digitifera & $A D$ & 2 & - \\
\hline Acropora millepora & AM & 9 & - \\
\hline Astreopora spp. & ASP & 4 & - \\
\hline Fungia scutaria & FS & 4 & - \\
\hline Favia spp. & FSP & 2 & - \\
\hline Madracis auretenra & MA & 2 & - \\
\hline Montastrea cavernosa & $\mathrm{MC}$ & 2 & - \\
\hline Porites asteroides & PAU & 7 & - \\
\hline Porites lobata & PL & 1 & - \\
\hline Mnemiopsis leidyi & ML & 19 & - \\
\hline Trichoplax adhaerens & TA & 10 & 14 \\
\hline Oscarella carmela & OC & 7 & - \\
\hline Drosophila melanogaster & $\mathrm{DM}$ & 1 & 16 \\
\hline Homo sapiens & HS & 7 & 18 \\
\hline Lottia gigantea & LG & 6 & 45 \\
\hline Octopus bimaculoides & OB & 3 & 20 \\
\hline Biomphalaria glabrata & BG & 5 & 21 \\
\hline Crassotrea gigas & CG & 5 & 31 \\
\hline Lingula unguis & LU & 2 & 12 \\
\hline Chlamydomonas reinhardtii & CR & 2 & 2 \\
\hline Ramazzotius varieornatus & RV & 6 & 15 \\
\hline Hypsibius dujardini & $\mathrm{HD}$ & 2 & 8 \\
\hline Strogylocentrotus purpuratus & SPU & 0 & 9 \\
\hline Helobdella robusta & HR & 0 & 15 \\
\hline Stichopus japonicus & SJ & 0 & 13 \\
\hline
\end{tabular}


Table 2.S2 Primer sequences with names and gene identification.

\begin{tabular}{|c|c|c|c|}
\hline Gene & Sequence ID & Primer Name & Primer Sequence \\
\hline \multirow{2}{*}{ gluR.1 } & 24461 & iGluR1f & GTGGGACGATCGAAGTGAATGT \\
\hline & 24461 & iGluR1r & GGAGTCTGTGTTTGTCTTCTGCC \\
\hline \multirow{2}{*}{ gluR.2 } & 24462 & iGluR2f & ACGTGAGGAAGGAGAGATTGA \\
\hline & 24462 & iGluR2r & AAGTGAAAAACCGGGCTGTG \\
\hline \multirow{2}{*}{ gluR23 } & 29220 & iGluR5f & GGAGCAATAGCAGAGGATGAC \\
\hline & 29220 & iGluR5r & CCGTAAGCTATGGTGAGAACAG \\
\hline \multirow{2}{*}{ gluR21 } & 25547 & iGluR6f & GTGTTGACTGTGATGCTAAATCC \\
\hline & 25547 & iGluR6r & CCACTTCAACGTCCAGTACAT \\
\hline \multirow{2}{*}{ gluR4 } & 5331 & iGluR7f & CTGATAGTCGCTGGAATCAATCT \\
\hline & 5331 & iGluR7r & CAGGACTCCATTCACCAATCTG \\
\hline \multirow{2}{*}{ gluR8 } & 29007 & iGluR8f & TGCAGTTCTTGATGCCCTTTGA \\
\hline & 29007 & iGluR8r & GACCAGAAGACGGCCTGATATAGA \\
\hline \multirow{2}{*}{110} & 29093 & L10f & CTTGGACAGGAGCATCGATAATA \\
\hline & 29093 & L10r & CCAGTGGGTTACGAAGACTTT \\
\hline \multirow{2}{*}{112} & 8348 & L12f & TCACATGGTGTCAGATGAGTATG \\
\hline & 8348 & L12r & CATACCAGTTTGGAGCCTATCA \\
\hline \multirow{2}{*}{ pabp } & 2230 & PABPf & AGAGAATTCCGCTTAGCAACTC \\
\hline & 2230 & PABPr & GAAGCAAGCTGTCGCTTAGA \\
\hline
\end{tabular}


Table 2.S3 Statistical significance of 2-way ANOVA between factors of time and treatment.

\begin{tabular}{|l|c|c|c|c|c|c|}
\hline \multicolumn{1}{|c|}{ Treatment } & gluR.1 & gluR.2 & gluR23+ & gluR21+ & gluR4 & gluR8 \\
\hline time & $0.033^{\star}$ & $0.0046^{\star}$ & $8.92 e-6^{\star}$ & $1.22 e^{*} 5^{\star}$ & 0.1734 & 0.1376 \\
\hline treatment & $0.0414^{\star}$ & $0.0016^{\star}$ & $0.0003^{\star}$ & $2.28 e-15^{\star}$ & 0.2428 & 0.5206 \\
\hline time:treatment & 0.2376 & 0.0839 & 0.1638 & $0.0190^{\star}$ & 0.4737 & 0.7197 \\
\hline
\end{tabular}

+ White's adjusted ANOVA for unequal variance, * significant $\mathrm{p}$-value $<0.05$ 
Table 2.S4 Statistical significance of 2-way ANOVA Tukey HSD post-hoc using 95\% family-wise confidence level on treatment factor.

\begin{tabular}{|c|c|c|c|c|c|c|}
\hline Treatment & gluR.1 & gluR.2 & gluR23+ & gluR21+ & gluR4 & gluR8 \\
\hline Serratia - Control & $0.0319^{*}$ & $0.0011^{*}$ & 0.9542 & 0.4907 & 0.4965 & 0.4916 \\
\hline Vibrio - Control & 0.4018 & $0.0746^{\prime}$ & 0.7376 & 0.7043 & 0.9160 & 0.9912 \\
\hline Vibrio - Serratia & 0.3854 & 0.2392 & 0.5569 & 0.1362 & 0.2830 & 0.4186 \\
\hline
\end{tabular}

+ White's adjusted ANOVA for unequal variance, * significant p-value $<0.05$ 


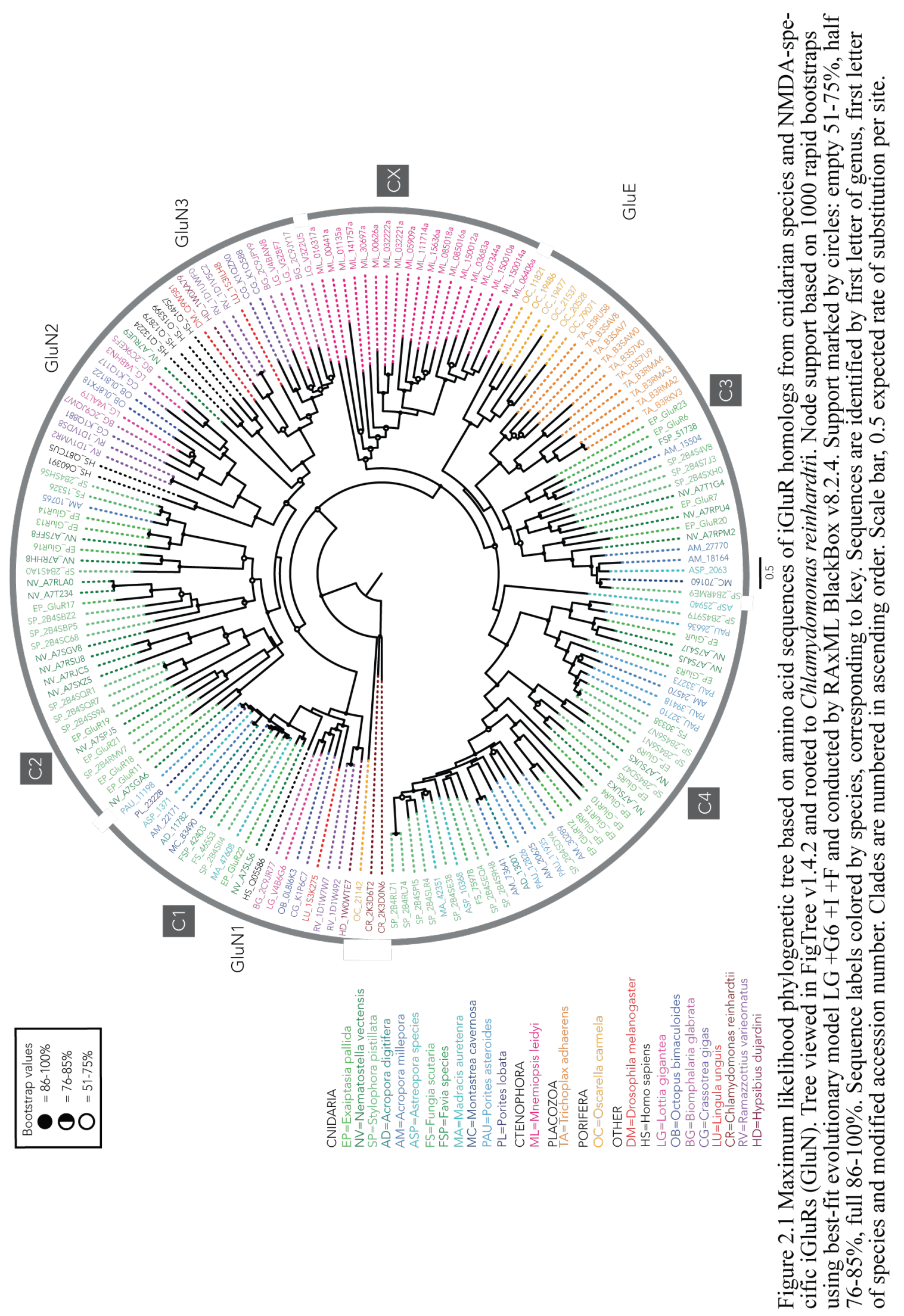




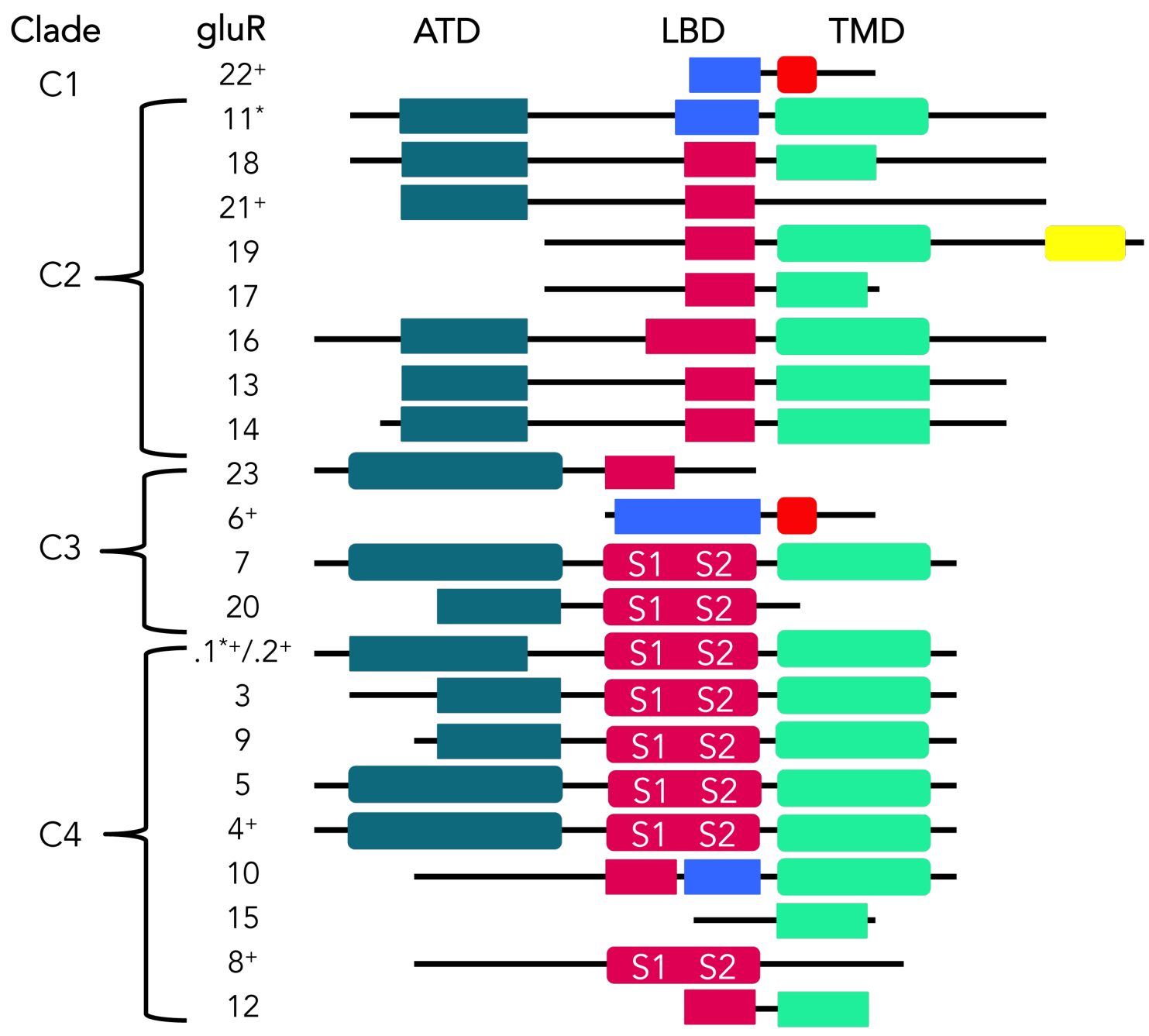

Figure 2.2 Conserved domains of Exaiptasia pallida ionotropic glutamate receptors, iGluRs. Amino- or N-terminal is ANF_receptor family ligand binding region (PF010904/IPR001828) colored turquoise. The ATD is known to not be conserved within IRs. The second conserved domain region is the ligand binding domain, usually specific for glutamate or glycine ligands. This region is colored a bright magenta if the conserved domain is ligand channel glutamate binding domain (PF10613/IPR019594) and contains conserved sequences of S1 and S2 if complete. This region is also conserved as bacterial extracellular solute-binding protein family 3 (PF00497/IPR001638), colored blue, and is closely related to the glutamate binding domain. The transmembrane region specific to ions for transmembrane channel is colored mint if it is the conserved sequence of the ligand channel domain ligand channel (PF00060/IPR001320). This region contains a conserved calmodulin-binding domain of NMDA receptor 1 subunit (PF 10562/IPR018882) for 22 and 6, colored red. There is one non-identified region colored yellow in 19. These colors correspond to colors of domains in Figures 2.3. GluR sequences are sorted in the order as present within the phylogenetic tree, ascending from the root. Complete domains have curved corners, partial sharp corners. Superscript $*$ denotes modeled in Figure 2.3. Superscript + denotes gene expression has been quantified in Figure 2.4. 


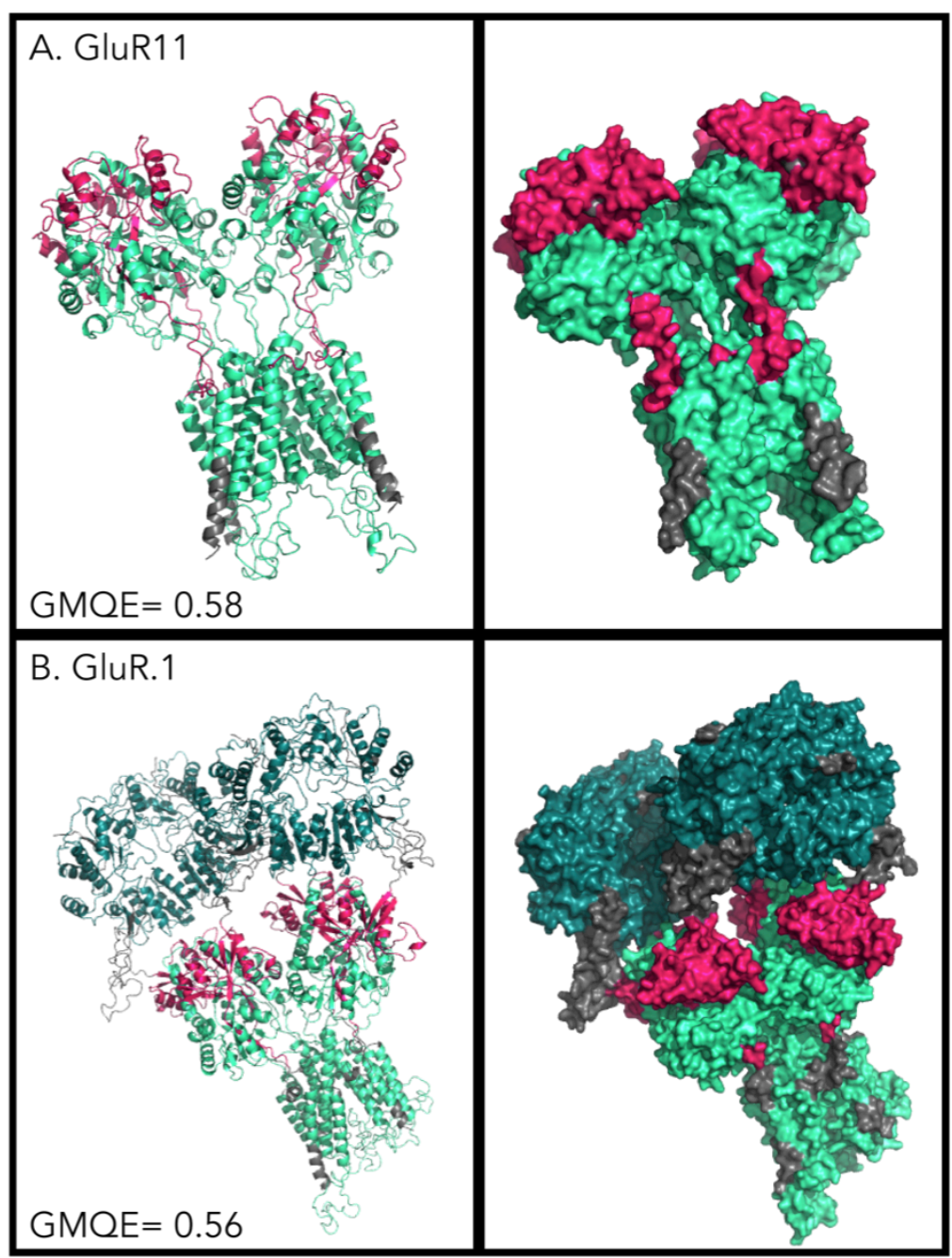

Figure 2.3 Homotetramers of Exaiptasia pallida GluR6 and GluR.1.

Predicted 3D structures (left) and predicted surface model (right) of selected sequences from cnidarian GluR11 (A) and GluR.1 (B). The protein is a tetramer with four subunits corresponding to one another. Predicted structures are colored by conserved domain regions (teal $=$ amino terminal domain, pink=ligand binding domain, green cyan=ion channel, grey=non-conserved). An extracellular N-terminal domain (NTD) is involved in assembly of iGluR subunits into heteromeric complexes. NTD precedes the ligand-binding domain (LBD), whose half-domains (S2 and S3) form a Venus flytrap-like structure that will close around glutamate and related ligands when activated. The transmembrane domain follows these two binding domains, forming a channel to allow calcium ions through the membrane. GluR11, as deduced from the amino acid sequence of E. pallida KXJ21128 aligned with Glutamate receptor, Voltage-dependent calcium channel gamma-2 subunit chimera (PDB ID $=5$ weo.1.A) and is modeled as a homotetramer (A, left). GluR11 predicted model has an overall QMEAN score of -4.93(A, middle). GluR.1 predicted structure has only ligand binding and ion channel conserved domains (A, right). GluR.1 protein, as deduced from the amino acid sequence of E. pallida KXJ14767 aligned with Glutamate Receptor 2 (PDB ID $=4 \mathrm{u} 2 \mathrm{p} .1$.B) and is modeled as a homotetramer (B, left). GluR.1 predicted model has an overall QMEAN score of -4.87 . GluR.1 predicted structure shows all three conserved iGluR domains, amino terminal, ligand binding site, ion channel (B, right). 


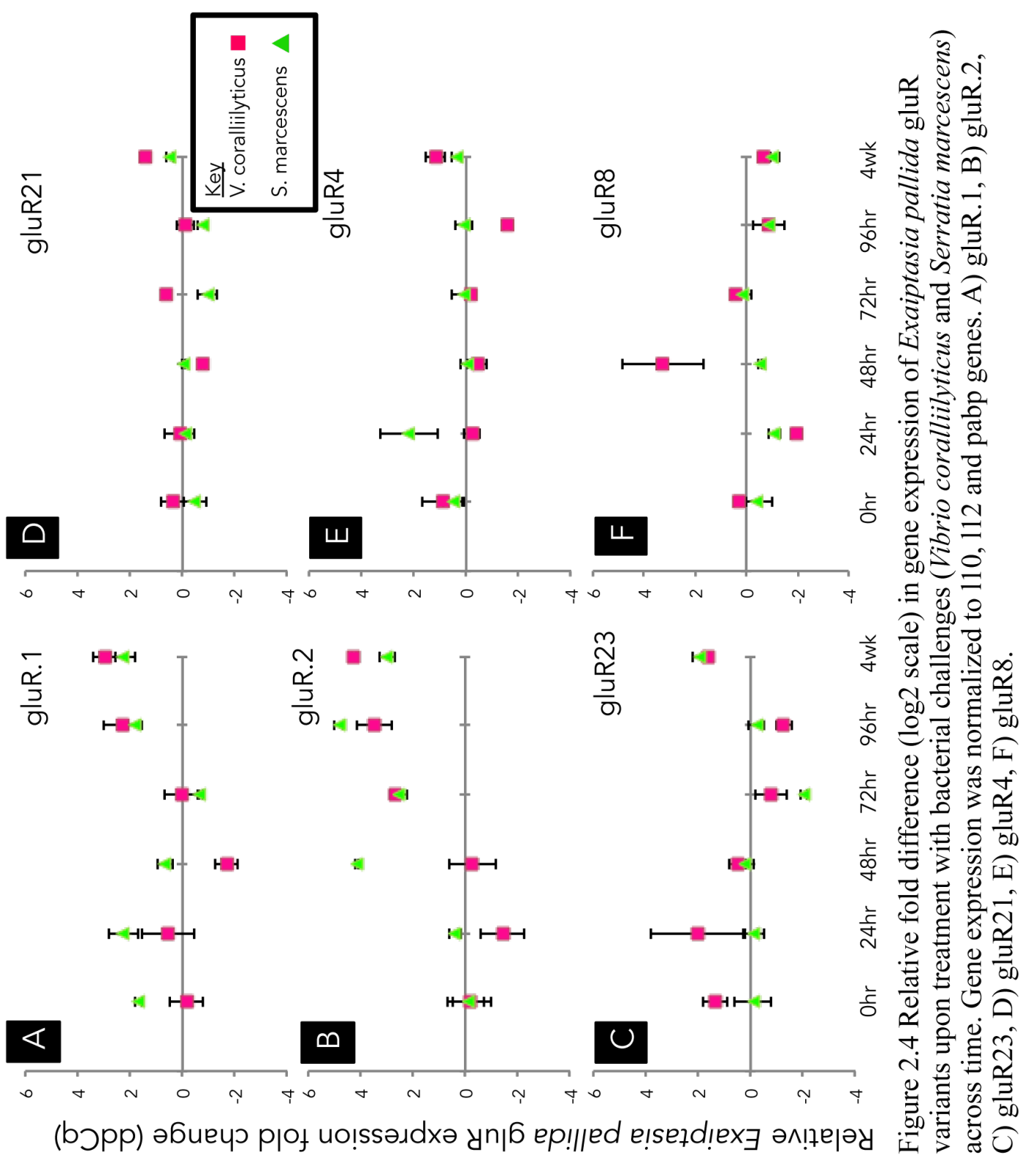




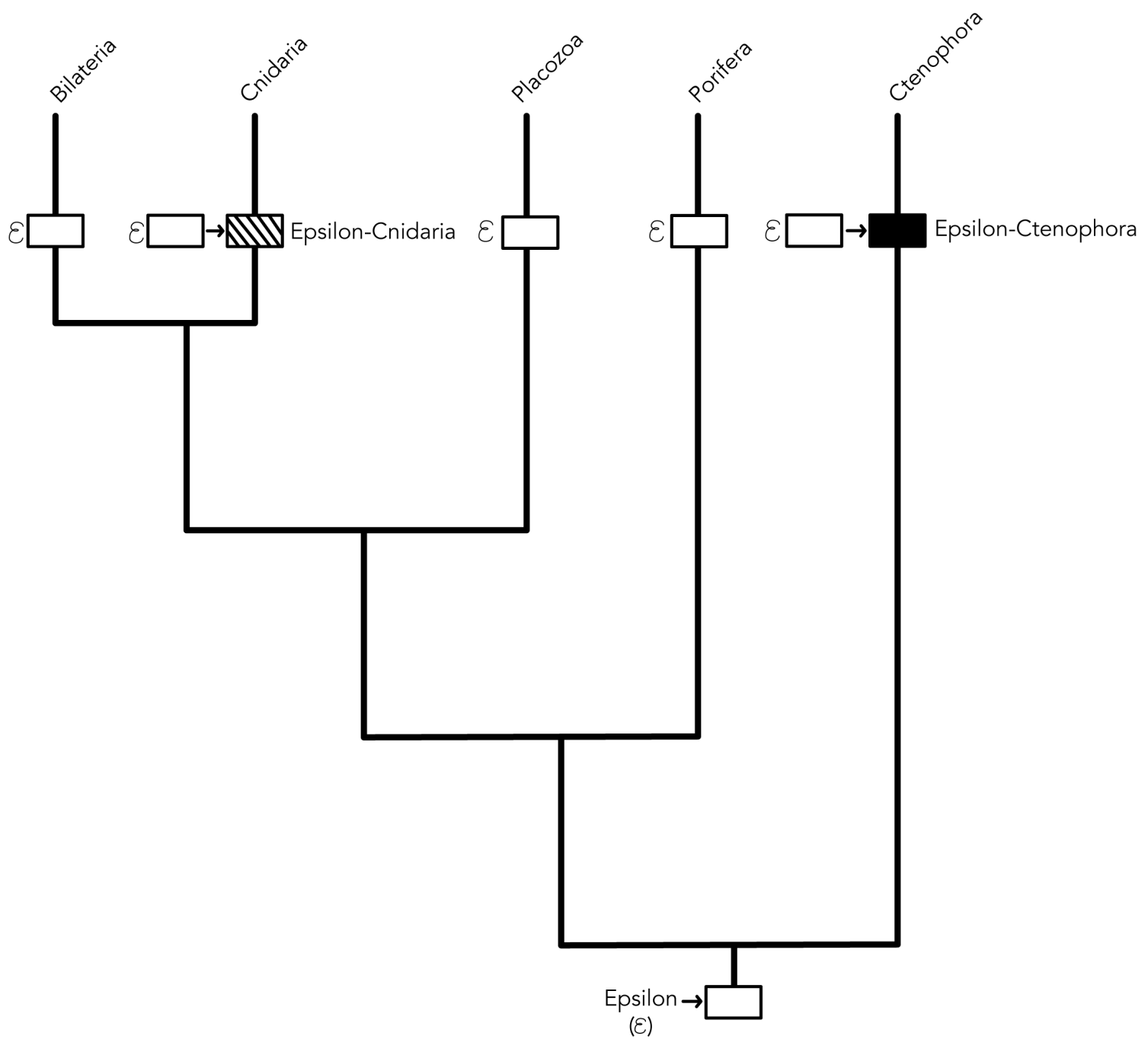

Figure 2.5 Proposed evolutionary tree for iGluR subfamily Epsilon to include cnidarian-specific divergent clade. 


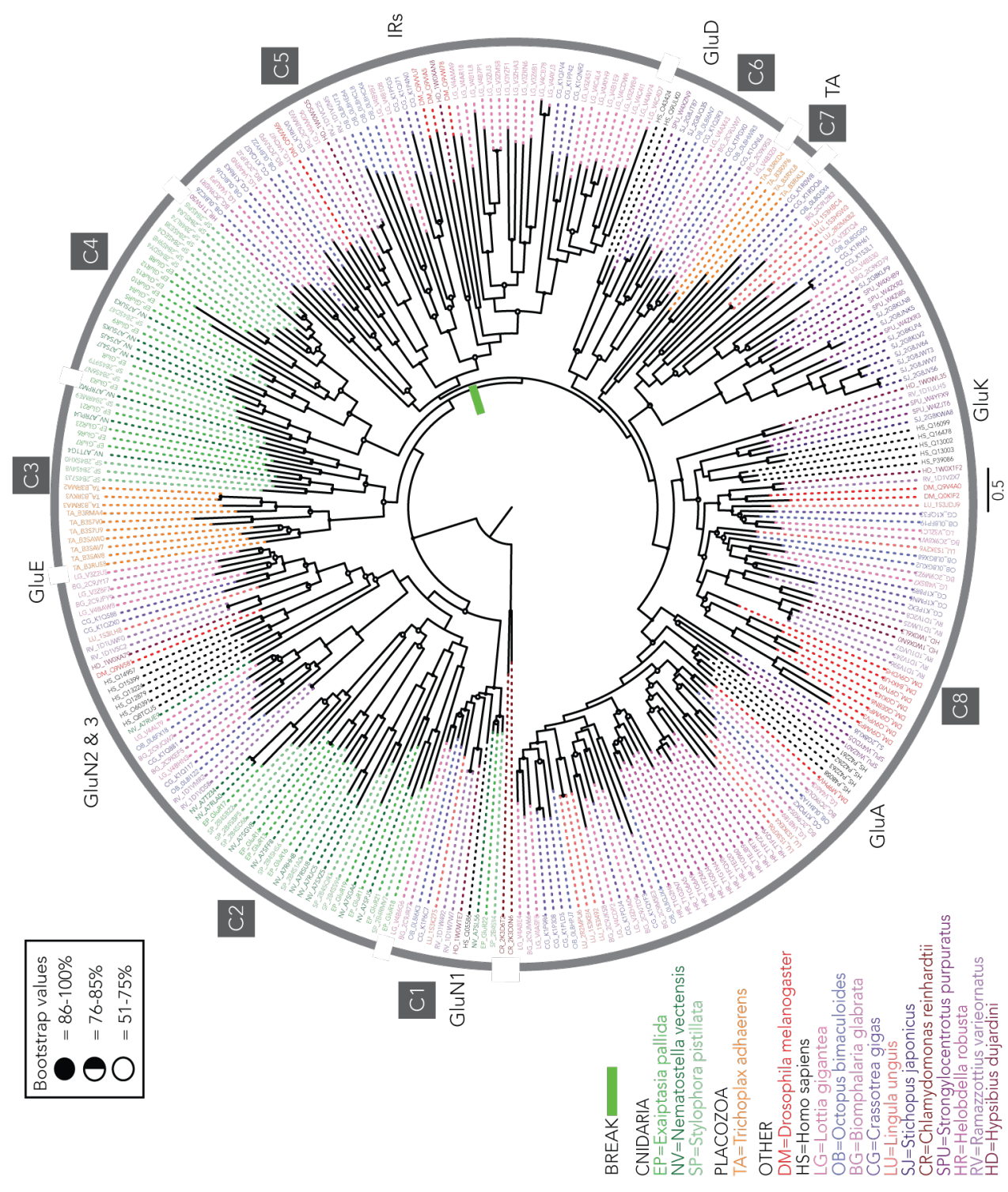

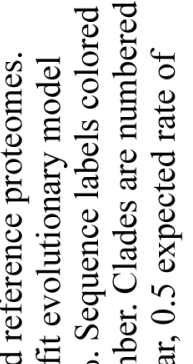

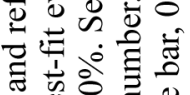
잉응 ¿ $\Xi$. :

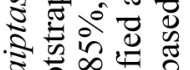

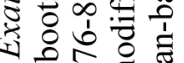

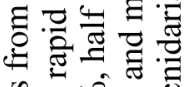
क 응응 出 言市的.

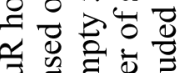
卷

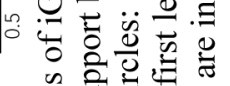
岂言.

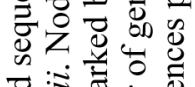
氞:

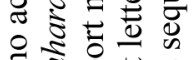

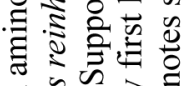

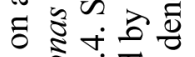
च요 옹

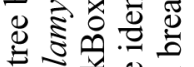

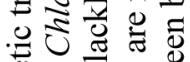

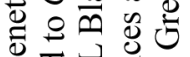

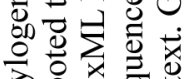
을 希昰 चृ छेष्ठ

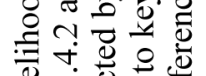
省要

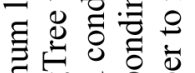

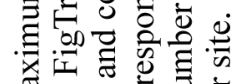

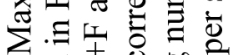

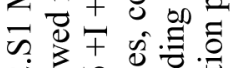

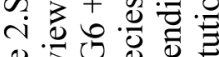

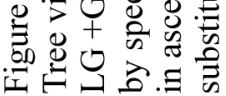




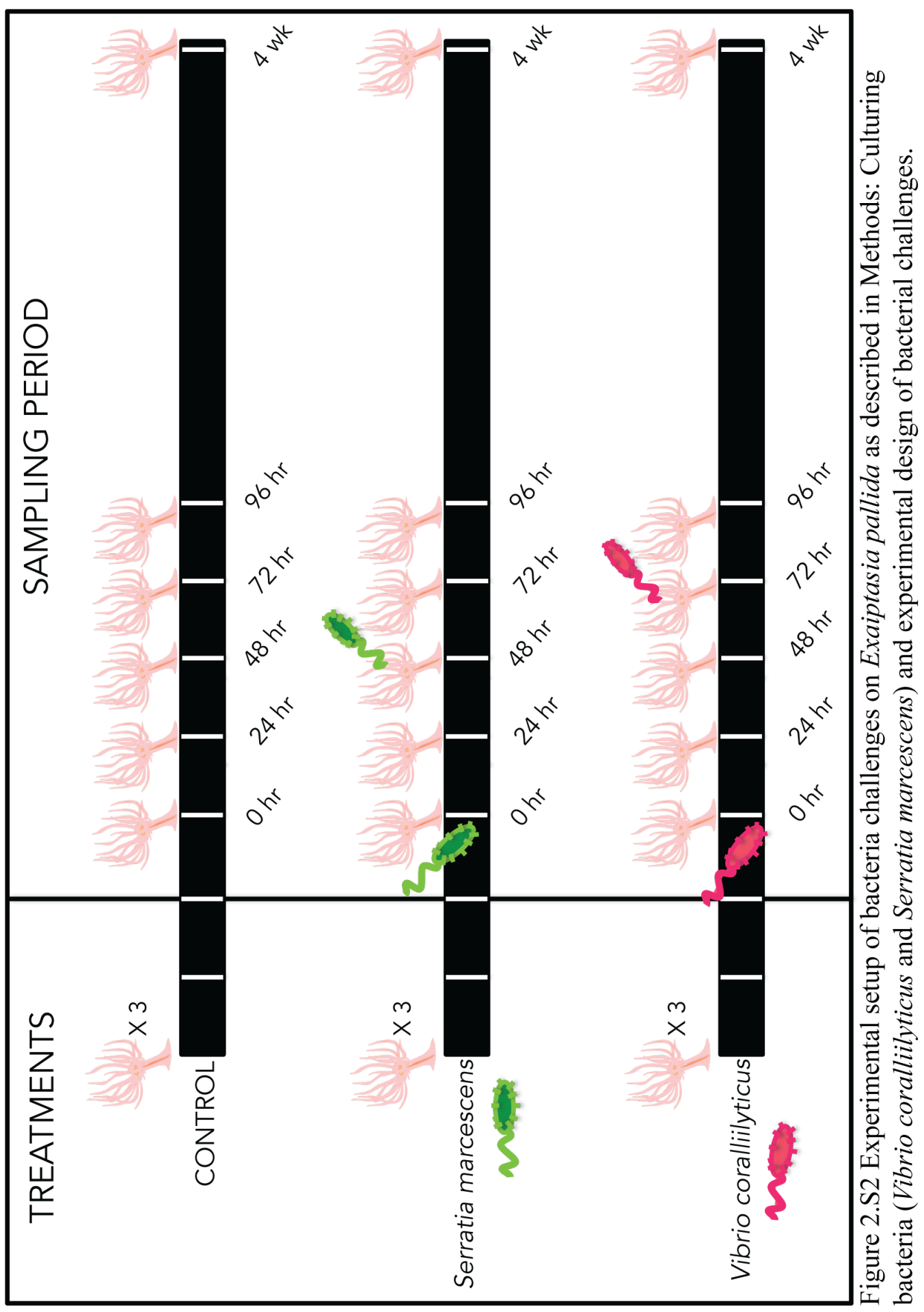




\subsection{References}

1. Seed KD, Lazinski DW, Calderwood SB, Camilli A. A bacteriophage encodes its own CRISPR/Cas adaptive response to evade host innate immunity. Nature. 2013;494(7438):489-91.

2. Seed KD. Battling Phages: How Bacteria Defend against Viral Attack. PLoS Pathog. 2015;11(6):e1004847.

3. Bosch TCG. Rethinking the role of immunity: lessons from Hydra. Trends Immunol. 2014;35(10):495-502.

4. Bosch TCG. Cnidarian-Microbe Interactions and the Origin of Innate Immunity in Metazoans. Annu Rev Microbiol. 2013;67:499-518.

5. Bosch TCG, Klimovich A, Domazet-Lošo T, Gründer S, Holstein TW, Jékely G, et al. Back to the Basics: Cnidarians Start to Fire. Trends Neurosci. 2017;40(2):92-105.

6. Hemmrich G, Miller DJ, Bosch TCG. The evolution of immunity : a low-life perspective. Trends Immunol. 2007;28(10).

7. Miller DJ, Hemmrich G, Ball EE, Hayward DC, Khalturin K, Funayama N, et al. The innate immune repertoire in Cnidaria - ancestral complexity and stochastic gene loss. Genome Biol. 2007;8(4):R59.

8. Rinkevich B. The "immunology trap" of anthozoans. ISJ. 2011;8:153-61.

9. Little TJ, Hultmark D, Read AF. Invertebrate immunity and the limits of mechanistic immunology. Nat Immunol. 2005;6(7):651-4.

10. Degnan SM. The surprisingly complex immune gene repertoire of a simple sponge, exemplified by the NLR genes: A capacity for specificity? Dev Comp Immunol. 2015;48(2):269-74.

11. Bosch TCG, Augustin R, Anton-Erxleben F, Fraune S, Hemmrich G, Zill H, et al. Uncovering the evolutionary history of innate immunity: The simple metazoan Hydra uses epithelial cells for host defence. Dev Comp Immunol. 2009;33:55969.

12. Janeway Jr. CA. Approaching the Asymptote? Evolution and Revolution in Immunology. Cold Spring Harb Symp Quant Biol. 1989;54:1-13. 
13. Medzhitov R. Approaching the Asymptote: 20 Years Later. Immunity. 2009;30(6):766-75.

14. Brown T, Bourne D, Rodriguez-Lanetty M. Transcriptional Activation of $\mathrm{c} 3$ and hsp70 as Part of the Immune Response of Acropora millepora to Bacterial Challenges. PLoS One. 2013;

15. Brown T, Rodriguez-Lanetty M. Defending against pathogens - immunological priming and its molecular basis in a sea anemone, cnidarian. Sci Rep. 2015;5:17425.

16. Forde BG, Roberts MR. Glutamate receptor-like channels in plants: a role as amino acid sensors in plant defence? F1000Prime Rep. 2014;6(37):1-7.

17. Forde BG. Glutamate signalling in roots. J Exp Bot. 2014;65(3):779-87.

18. Benton R, Vannice KS, Gomez-Diaz C, Vosshall LB. Variant Ionotropic Glutamate Receptors as Chemosensory Receptors in Drosophila. Cell. 2009;136(149-162).

19. Benton R. Multigene Family Evolution: Perspectives from Insect Chemoreceptors. Trends Ecol Evol. 2015;30(10):590-600.

20. Abuin L, Bargeton B, Ulbrich MH, Isacoff EY, Kellenberger S, Benton R. Functional Architecture of Olfactory Ionotropic Glutamate Receptors. Neuron. 2011;69:44-60.

21. Croset V, Rytz R, Cummins SF, Budd A, Brawand D, Kaessmann H, et al. Ancient protostome origin of chemosensory ionotropic glutamate receptors and the evolution of insect taste and olfaction. PLoS Genet. 2010;6(8):e1001064.

22. Hart AC, Chao MY. From odors to behaviors in Caenorhabditis elegans. The Neurobiology of Olfaction. Front Neurosci. 2010;

23. Rytz R, Croset V, Benton R. Ionotropic Receptors (IRs): Chemosensory ionotropic glutamate receptors in Drosophila and beyond. Insect Biochem Mol Biol. 2013;43:888-97.

24. Liebeskind BJ, Hofmann HA, Hillis DM, Zakon HH. Evolution of Animal Neural Systems. Annu Rev Ecol Evol Syst. 2017;48:377-98.

25. Putnam NH, Srivastava M, Hellsten U, Dirks B, Chapman H, Salamov A, et al. Sea Anemone Genome Reveals Ancestral Eumetazoan Gene Repertoire and Genomic Organization. Science (80- ). 2007;317(5834):86-94. 
26. Ryan JF, Pang K, Schnitzler CE, Nguyen A-D, Moreland RT, Simmons DK, et al. The genome of the ctenophore Mnemiopsis leidyi and its implications for cell type evolution. Science (80- ). 2013;342(6164):1242592.

27. Moroz LL, Kocot KM, Citarella MR, Dosung S, Norekian TP, Povolotskaya IS, et al. The ctenophore genome and the evolutionary origins of neural systems. Nature. $2014 ; 510$.

28. Watanabe H, Fujisawa T, Holstein TW. Cnidarians and the evolutionary origin of the nervous system. Dev Growth Differ. 2009;51:167-83.

29. Pierobon P. Coordinated modulation of cellular signaling through ligand-gated ion channels in Hydra vulgaris (Cnidaria, Hydrozoa). Int J Dev Biol. 2012;56(68):551-65.

30. Jékely G. Origin and early evolution of neural circuits for the control of ciliary locomotion. Proc R Soc B. 2011;278:914-22.

31. Vatsa P, Chiltz A, Bourque S, Wendehenne D, Garcia-brugger A, Pugin A. Biochimie Involvement of putative glutamate receptors in plant defence signaling and NO production. Biochimie. 2011;93(12):2095-101.

32. Manzoor H, Kelloniemi J, Chiltz A, Wendehenne D, Pugin A, Poinssot B, et al. Involvement of the glutamate receptor AtGLR3.3 in plant defense signaling and resistance to Hyaloperonospora arabidopsidis. Plant J. 2013;76(3):466-80.

33. Tapken D, Anschütz U, Liu L, Huelsken T, Seebohm G, Becker D, et al. A Plant Homolog of Animal Glutamate Receptors Is an Ion Channel Gated by Multiple Hydrophobic Amino Acids. Sci Signal. 2013;6(279):ra47.

34. Baumgarten S, Simakov O, Esherick LY, Liew YJ, Lehnert EM, Michell CT, et al. The genome of Aiptasia, a sea anemone model for coral symbiosis. Proc Natl Acad Sci. 2015;Early Edit.

35. Zhang Z, Schwartz S, Wagner L, Miller W. A Greedy Algorithm for Aligning DNA Sequences. J Comput Biol. 2000;7(12):203-14.

36. Lefort V, Longueville JE, Gascuel O. SMS: Smart Model Selection in PhyML. Mol Biol Evol. 2017;34(9):2422-4.

37. Miller MA, Pfeiffer W, Schwartz T. Creating the CIPRES Science Gateway for Inference of Large Phylogenetic Trees. 2010; 
38. Guindon S, Dufayard JF, Lefort V, Anisimova M, Hordijk W, Gascuel O. New algorithms and methods to estimate maximum-likelihood phylogenies: Assessing the performance of PhyML 3.0. Syst Biol. 2010 May;59(3):307-21.

39. Finn RD, Bateman A, Clements J, Coggill P, Eberhardt RY, Eddy SR, et al. Pfam: the protein families database. Nucleic Acids Res. 2014;42(Database issue):D22230.

40. Arnold K, Bordoli L, Rgen Kopp J, Schwede T. The SWISS-MODEL workspace: a web-based environment for protein structure homology modelling. Bioinformatics. 2006;22(2):195-201.

41. Guex N, Peitsch MC, Schwede T. Automated comparative protein structure modeling with SWISS-MODEL and Swiss-PdbViewer: A historical perspective. Electrophoresis. 2009 Jun 1;30(S1):S162-73.

42. Bertoni M, Kiefer F, Biasini M, Bordoli L, Schwede T. Modeling protein quaternary structure of homo- and hetero-oligomers beyond binary interactions by homology. Sci Rep. 2017 Dec 5;7(1):10480.

43. Kiefer F, Arnold K, Kü Nzli M, Bordoli L, Schwede T. The SWISS-MODEL Repository and associated resources. Nucleic Acids Res. 2009;37(Database issue):D387-92.

44. Biasini M, Bienert S, Waterhouse A, Arnold K, Studer G, Schmidt T, et al. SWISS-MODEL: modelling protein tertiary and quaternary structure using evolutionary information. Nucleic Acids Res. 2014;42:W252-8.

45. Benkert P, Biasini M, Schwede T. Toward the estimation of the absolute quality of individual protein structure models. Bioinformatics. 2011 Feb 1;27(3):343-50.

46. Waterhouse A, Bertoni M, Bienert S, Studer G, Tauriello G, Gumienny R, et al. SWISS-MODEL: homology modelling of protein structures and complexes. Nucleic Acids Res. 2018 Jul 2;46(W1):W296-303.

47. Bienert S, Waterhouse A, de Beer TAP, Tauriello G, Studer G, Bordoli L, et al. The SWISS-MODEL Repository - new features and functionality. Nucleic Acids Res. 2017 Jan 4;45(D1):D313-9.

48. Remmert M, Biegert A, Hauser A, Söding J. HHblits: lightning-fast iterative protein sequence searching by HMM-HMM alignment. Nat Methods. 2012 Feb $25 ; 9(2): 173-5$.

49. Lewis ACF, Jones NS, Porter MA, Deane CM. What Evidence Is There for the Homology of Protein- Protein Interactions? PLoS Comput Biol. 2012;8(9). 
50. Benkert P, Tosatto SCE, Schomburg D. QMEAN: A comprehensive scoring function for model quality assessment. Proteins Struct Funct Bioinforma. 2008;71:261-77.

51. The PyMOL Molecular Graphics System. Schrödinger, LLC;

52. Brown T. Phenomenological and Molecular Basis of the Cnidarian Immune System. Florida International University; 2017.

53. Gimbrone N, Castro H, Ritchie KB, Teplitski M, Alagely A, Paul VJ, et al. Interactions between the tropical sea anemone Aiptasia pallida and Serratia marcescens, an opportunistic pathogen of corals. Environ Microbiol Rep. 2014;6(3):287-92.

54. Aranda PS, Lajoie DM, Jorcyk CL. Bleach Gel: A Simple Agarose Gel for Analyzing RNA Quality. Electrophoresis. 2012;33(2):366-9.

55. Ruijter JM, Pfaffl MW, Zhao S, Spiess AN, Boggy G, Blom J, et al. Evaluation of qPCR curve analysis methods for reliable biomarker discovery: Bias, resolution, precision, and implications. Methods. 2013;59(1):32-46.

56. Hellemans J, Mortier G, Paepe A, Speleman F, Vandesompele J. qBase relative quantification framework and software for management and automated analysis of real-time quantitative PCR data. Genome Biol. 2007;8(2):R19.

57. Vandesompele J, De Preter K, Pattyn ilip, Poppe B, Van Roy N, De Paepe A, et al. Accurate normalization of real-time quantitative RT-PCR data by geometric averaging of multiple internal control genes. Genome Biol. 2002;3(7):0034.1-12.

58. R Core Team. R: A Language and Environment for Statistical Computing. Vienna, Austria: R Foundation for Statistical Computing; 2014.

59. Royston JP. An Extension of Shapiro and Wilk's W Test for Normality to Large Samples. Source J R Stat Soc Ser C (Applied Stat. 1982;31(2):115-24.

60. Hester J. glue Interpreted String Literals for R: An implementation of Interpreted String Literals (f-strings) for R.

61. Anokhin B, Hemmrich-Stanisak G, Bosch T. Karyotyping and single-gene detection using fluorescence in situ hybridization on chromosomes of Hydra magnipapillata (Cnidaria: Hydrozoa). Comp Cytogenet. 2010 Dec 30;4(2):97-110. 
62. Mangiafico SS. Summary and Analysis of Extension Program Evaluation in R. version 1. rcompanion.org/handbook/, editor. New Brunswick, NJ: Rutgers Cooperative Extension; 2016.

63. Peters G. userfriendlyscience: Quantitative analysis made accessible. https://userfriendlyscience.com.; 2018.

64. Collingridge GL, Olsen RW, Peters J, Spedding M. A nomenclature for ligandgated ion channels. Neuropharmacology. 2009;56(1):2-5.

65. Lehnert EM, Burriesci MS, Pringle JR. Developing the anemone Aiptasia as a tractable model for cnidarian-dinoflagellate symbiosis: the transcriptome of aposymbiotic $A$. pallida. BMC Genomics. 2012;13:271.

66. Hayashi T. Evolutionarily conserved palmitoylation-dependent regulation of ionotropic glutamate receptors in vertebrates. Neurotransmitter. 2014 Jan;1(e388).

67. Greer JB, Khuri S, Fieber LA. Phylogenetic analysis of ionotropic L- glutamate receptor genes in the Bilateria, with special notes on Aplysia californica. BMC Evol Biol. 2017;17(11).

68. Wang Y, Liu Q, Dai W, Sun Z, Zhou L, Zhang J, et al. Evolutionary Mode and Functional Divergence of Vertebrate NMDA Receptor Subunit 2 Genes. PLoS One. 2010;5(10):e13342.

69. Ramos-Vicente D, Ji J, Gratacòs-Batlle E, Gou G, Reig-Viader R, Luís J, et al. Metazoan evolution of glutamate receptors reveals unreported phylogenetic groups and divergent lineage-specific events. Elife. 2018;7:e35774.

70. Koo JCP, Hampson DR. Phylogenic and Evolutionary Analysis of Glutamate Receptors Based on Extant Invertebrate Genes. J Undergrad Life Sci. 2010;4(1):42-9.

71. Eyun S-I, Soh HY, Posavi M, Munro JB, Hughes DST, Murali SC, et al. Evolutionary History of Chemosensory-Related Gene Families across the Arthropoda. Mol Biol Evol. 2017;34(8):1838-62.

72. Lynch M. The Evolutionary Fate and Consequences of Duplicate Genes. Science (80- ). 2002;290(5494):1151-5.

73. Force A, Lynch M, Pickett FB, Amores A, Yan YL, Postlethwait J. Preservation of duplicate genes by complementary, degenerative mutations. Genetics. 1999;151(4):1531-45. 
74. Rastogi S, Liberles D. Subfunctionalization of duplicated genes as a transition state to neofunctionalization. BMC Evol Biol. 2005;5(28).

75. Glezer I, Zekki H, Scavone C, Rivest S. Modulation of the innate immune response by NMDA receptors has neuropathological consequences. J Neurosci. 2003;23(35):11094-103.

76. Boldyrev AA, Carpenter DO, Johnson P. Emerging evidence for a similar role of glutamate receptors in the nervous and immune systems. J Neurochem. 2005;95:913-8.

77. Ganor Y, Levite M. The neurotransmitter glutamate and human T cells: glutamate receptors and glutamate-induced direct and potent effects on normal human $\mathrm{T}$ cells, cancerous human leukemia and lymphoma T cells, and autoimmune human T cells. J Neural Transm. 2014;121(8):983-1006. 


\section{CHAPTER III. EXPRESSION OF CHEMOSENSORY IONOTROPIC GLUTAMATE RECEPTORS UNDER THE INFLUENCE OF BIOLOGICAL AND ENVIRONMENTAL OSCILLATIONS IN CNIDARIANS}

\subsection{Abstract}

Environmental fluctuations of light, tide, and temperature influence physiology and behavior of marine invertebrates, particularly sessile corals and anemones.

Accompanying environmental changes is the internal endosymbiosis of algal dinoflagellates, which dominantly affects physiology of cnidarian hosts through diel rhythms. Both intracellular and extracellular oscillations have been demonstrated to alter host gene expression within corals and anemones, including products of metabolic processing. Chemosensory gene regulation has not been explored in relation to diurnal fluctuations and endosymbiosis. In Cnidaria, the chemosensory protein family, ionotropic glutamate receptors (iGluRs), are of particular consequence as signaling proteins in interpreting envrionmental cues. Here we quantified mRNA expression of two Exaiptasia pallida iGluR genes (gluR.2 and gluR8) to compare the influence of a recurring light: dark cycle and response to constant darkness on gene transcription. In the absence of light, periodicity length of E. pallida gluR.2 and gluR8 expression increased. The change in transcription periodicity as result of light changes paired with symbiotic versus aposymbiotic periodicity changes from Exaiptasia diaphana transcriptomic data, implicates regulation of iGluRs by Symbiodiniacea metabolic outputs. While an endogenous circadian rhythm does not appear to be the primary contributor to 
biorhythmic expression cycling in these chemosensory genes, symbiosis and diurnal fluctuations appear to have a role.

\subsection{Keywords}

Exaiptasia; chemosensing; iGluR; circadian; diurnal; diel; circatidal; cnidarian; symbiosis

\subsection{Introduction}

Corals build the highly productive framework of diversified reef ecosystems that provide essential habitat for fish and marine invertebrates, a foundational service for fisheries and ecotourism (1-3). Corals and closely related anemones are particularly impacted by environmental diel cycles of light and temperature because they are sessile (4-6). While both diel and circadian refer to a period of approximately 24-hrs, circadian rhythms are considered as endogenous, oscillating under constant conditions in absence of a stimulus whereas in diel rhythms are directed by external cues $(7,8)$. There are also observations of diel rhythms for a suite of cnidarian behaviors and variation in gene expression (9-12). Daily light cycles regulate numerous physiological processes and behaviors, observed in wide-ranging physiological and even behavioral changes of coral from metabolism to tentacle retraction and expansion (13-16). Evidence of altered expression of genes related to growth is also consistent with a diurnal response $(5,17)$. Light drives much of the physiological responses observed through the innerworkings between symbiosis and metabolysis. 
Cnidarian diel cycles are strongly linked to central metabolism and cellular redox states through glycolytic enzyme cycles (12). Within symbiotic cnidarians, diurnal cycles produce alternating energy sources from nutrients delivered by a close-knit endosymbiosis with the algal dinoflagellate family Symbiodiniaceae $(3,12,18-22)$. In daylight, energy is derived from photosynthesis by the Symbiodiniacea partner alongside consequent reactive oxygen species (ROS) production, while at night corals consume zooplankton (23-29). Heterotrophic feeding and continued respiration in the absence of photosynthesis can lead to hypoxia and decreased $\mathrm{pH}$ within tissues $(12,24,30,31)$. A predicted consequence of nightly respiration-driven hypoxia is the upregulation of hypoxia inducible factor (HIF)-targeted genes i.e., glycolic enzymes aldolase, phosphoglycerate kinase, and phosphoglycerate dehydrogenase (32). The characteristic 24-hr periodicity occurred in facultative symbiotic anemones (32). It it proposed that the endosymbiosis of Symbiodiniaceae promotes the cycle of a diel or circadian 24-hr period (33).

The origins of circadian clockwork within cnidarians are suggested to include conserved molecular and functional components $(10,15,16,33,34)$. These molecular components follow the 24-hr circadian cycle, but have two alternative regulating sources. Cnidarian clock, cycle, $(11,15)$ and cryptochrome $(14)$ are proposed to act as clockcontrolled genes (CCG), meaning expression is regulated by an internal molecular clock to produce measureable circadian rhythm as a cycle (16). In contrast, diel cycle genes (DCGs) display a 24-hr period, but remain reliant on consistent diel oscillations. Cnidarian DCGs include some cryptochrome (blue light photoreceptor) genes (14). Select 
photoreceptor DCGs i.e., cryptochromes and opsins, are hypothesized to interact with circadian genes as transcription factors to produce a response via physiological changes under circadian regulation $(10,12,15,16,32)$. It appears these biogeochemical environmental conditions are the ultimate drivers behind physiological changes in molecular systems through entrainment of physiological rhythms. However, the reception and propogation of signals within the response to environmental cues in cnidarians is largely unknown and predominantly based on correlative findings.

Cnidarians have chemosensory receptors coded within their genomes to sense environmental changes $(35,36)($ Ch.2). Receptors include chemosensory gated ion channels that have the potential to propogate further signals. The expression rhythms associated with chemosensory genes that are responsible for near immediate reactions and responses, including the family of ionotropic glutamate receptors (iGluRs), are for the most part unknown. The function of iGluRs has yet to be identified within cnidarians and most organisms, aside from antennal ionotropic receptors within insects (37-40). These transmembrane proteins receive exogenous chemical signals and relay signals through a membrane-bound ion channel $(39,41)$. Signals sent through the iGluR prompt responses in accordance with chemical environment changes, either as synaptic communication or feeding detection (42-45). The family of iGluRs have established roles within neuronal communication (46-48) and the potential for relaying signals in perpetuating biological rhythms. Genes regulated by biological rhythms are predicted to change expression in anticipation of environmental oscillations $(15,36,49,50)$. Initial measurements of iGluR expression within the sea anemone Exaiptasia pallida (Agassiz 
in Verrill, 1864) indicated variable expression responses to pathogenic bacteria; unknown if variation in gluR expression is the result of biological rhythms (Fig. 3.1; Ch. 2). This opened the possibility that variation in gluR expression reflects a biological rhythm. Examination of expression profiles further prompted exploration into the rhythmicity of chemosensory genes to determine if the circadian clock or another rhythm regulates gluR expression. Responses to environmental variations were previously reported in chemosensory iGluR, Ionotropic Receptor 25a (IR25a) (51). Clock protein oscillations are postulated to control temperature-dependent behaviors in low-amplitude envrionmental cycles within central clock neurons in conjunction with cycling expression of IR25a in Drosophila $(51,52)$. Similar low-varying day-night fluctuations are found in some marine environments during certain seasons $(3,6)$. We postulate intracellular cycles further perpetuate the influence of expression cycles via environmental, chemosensory receptors.

The chemosensory iGluR family includes receptors that respond to glutamate as an activating ligand (53). Cnidarian glutamate cycling, within cells, is observed in the conversion of glutamine to glutamate via glutamate synthase at night (Fig. 3.2) (5). Glutamine synthetase in E. pallida is predicted to fix inorganic nitrogen as a function of Symbiodiniacea-cnidarian endosymbiosis $(54,55)$. The rhythm of gluR genes may be consequence of diurnal cycling or an endogenous source. In order to test the diel cycle and whether expression is influenced by diurnal factors or if expression has an endogenous rhythm, E. pallida anemones were subjected to two diel treatments to compare consequent gene expression of gluRs. To follow a circadian cycle, a peak and 
trough would be clearly present for E. pallida gluR gene expression within both a control treatment and retained through a 48-hr period of darkness. However, another biological rhythm may be apparent if there is an endogenous expression regulator as it is possible gluR expression patterns are associated with clock genes (9). Alternatively, if gluR expression is driven by documented daily changes in glutamate metabolism $(5,53,55)$, expression patterns would be expected to decrease at the end of day to middle of night. Expression of gluR genes may be sensitive to changing glutamate levels from Symbiodiniacea metabolism, which is predicted to lead to differentiating expression cycles between diel cycling (4). Hypotheses on the function of E. pallida gluR genes can be challenged through measuring the gene expression responses between the two diurnal states.

\subsection{Methods}

3.4.1 Animal maintenance: Clonal symbiotic E. pallida anemones (CC7) were used in the experiment to reduce genetic variation and maintained in artificial seawater at approximately $27^{\circ} \mathrm{C}$. Populations were maintained in a day/night cycle of $12 \mathrm{hr}$ light: 12 hr dark with 40 to $60 \mu \mathrm{mol}$ photons $\mathrm{m}^{-2} \mathrm{~s}^{-1}$ of light intensity and fed freshly hatched brine shrimp Artemia nauplii twice per week. E. pallida anemones were approximately $3 \mathrm{~mm}$ in diameter and $8 \mathrm{~mm}$ in length.

3.4.2 Diurnal experimentation: Two light treatments were used to test how the diurnal light cycle affects gluR.2 (isoform of GluR) and gluR8 expression. Clonal CC7 symbiotic 
anemones were placed into 12 -well plates $72 \mathrm{hrs}$ prior to sampling for settlement and not fed throughout the experiment. Control anemones remained under $12 \mathrm{hr}$ light: $12 \mathrm{hr}$ dark (LD) with lights turning on at $0800 \mathrm{hrs}$ and off at $2000 \mathrm{hrs}$ (Fig. 3.3). In the DD treatment, anemones were placed in the dark for one complete $24-\mathrm{hr}$ cycle prior to sampling to study if expression in temporal, displaying circadian rhythmic periodicity or diel influence without carry over from the first 24 hrs (Fig. 3.3) (56). Anemones were snap-frozen using liquid nitrogen every four hours for a $24 \mathrm{hr}$ period (Fig. 3.3). The process of placing anemones in well plates and consequent LD and DD sampling was repeated four additional times, over the course of four weeks to collect a total of five biological samples per time point for each cycle treatment (Fig. 3.3) (56).

\subsubsection{RNA extraction and cDNA synthesis: We used the Monarch Total RNA Miniprep} Kit (New England Biolabs, Ipswich, MA) to extract RNA. The RNA was eluted in a final volume of $50 \mu \mathrm{L}$ of nuclease-free water. Quantification of extracted RNA was conducted using a NanoDrop 2000 (ThermoFisher, Carlsbad, CA) and RNA integrity assessed visually on a $1 \%$ agarose gel, including 11 samples randomly selected for BioAnalyzer (Agilent Technologies, Inc, Waldbronn, Germany) assessments. Three of the 60 samples with initial spectrophotometric $260 / 230$ ratio of less than 1.5 underwent additional cleanup using 10\% $3 \mathrm{M}$ sodium acetate ( $\mathrm{pH}$ 5.2), RNA-grade glycogen at a final concentration of $0.05-1.0 \mu \mathrm{g} / \mathrm{mL}$, and 2.5 volumes of $100 \% \mathrm{EtOH}$ to the sample. Following precipitation overnight at $-80{ }^{\circ} \mathrm{C}$, samples were centrifuged for $20 \mathrm{~min}$ at $9500 \mathrm{rpm}\left(4^{\circ} \mathrm{C}\right)$ to pellet RNA. The pellet was washed with $250 \mu \mathrm{L}$ of $70 \% \mathrm{EtOH}$, centrifuged for $5 \mathrm{~min}$ at the same conditions listed above, and dried before re-suspending 
in $30 \mu \mathrm{L}$ of nuclease-free water. Synthesis of cDNA was performed with Superscript III first strand synthesis system (Life Technologies, Eugene, OR) using $200 \mathrm{ng}$ of RNA and Oligo dT primer.

3.4.4 Quantitative RealTime-PCR assays: To measure RNA expression using $\mathrm{qPCR}$, a master mix of SsoAdvanced Universal SYBR Green Supermix (BioRad, Hercules, CA), $0.34 \mu \mathrm{M}$ of forward and reverse primers and nuclease-free water was added in $19.5 \mu \mathrm{L}$ aliquots to each well with $0.5 \mu \mathrm{L}$ of sample template. Primers with a length of 18 to 25 base pairs and an optimal melting temperature $(\mathrm{Tm})$ of $60{ }^{\circ} \mathrm{C}$ were designed using PrimerQuest (IDT, Coralville, IA). Through comparing to the E. pallida genome (Baumgarten et al., 2015), primer pairs (Table 3.1) were designed across introns to prevent genomic DNA amplification. Primer sets for gluR.2 and gluR8 were verified by length using normal endpoint PCR. Primer concentrations were optimized by comparing qPCR reads using $0.5 \mathrm{nM}$ and $0.25 \mathrm{nM}$ of primer in order to minimize primer dimer formation and maximize target amplification.

Plates for each gene were run on Bio-Rad CFX Connect systems (BioRad, Hercules, CA) at a thermocycle of initial polymerase activation of $30 \mathrm{~s}$ at $98{ }^{\circ} \mathrm{C}$, followed by 40 cycles of $15 \mathrm{~s}$ denaturation at $95{ }^{\circ} \mathrm{C}$, then annealing for $30 \mathrm{~s}$ at $54{ }^{\circ} \mathrm{C}$, followed by extension for $30 \mathrm{~s}$ at $72{ }^{\circ} \mathrm{C}$ with a plate read, and after 40 cycles a final Melt-Curve analysis. Plates had an Inter-Run Calibrator to control for variation of reads between runs. Negative controls for No Reverse Transcriptase were run for all samples with primers to confirm no genomic DNA contamination or bias. Negative controls for 
primers and samples were also run on plates to confirm no bias or contamination. Raw output was corrected for variations between runs with an inter-plate calibrator $(57,58)$. Gene expression was normalized using the geometric means of three reference genes, ribosomal genes 110 and 112, and poly (A)-binding protein, which had minimal change in expression among treatments or time points (59-64). Relative quantities were calculated by subtracting the minimum normalized average from each biological replicate to measure relative difference in expression (65-67).

Expression quantities were tested for normality, variance, heteroscedasticity using the gvlma package (68) and Shapiro-Wilk test $(69,70)$ in Rv3.5.0 (71). Statistical analyses to compare light cycle effects on relative quantities of GluR.2 across time points were completed by linear model ANOVA using Tukey Honestly Significant Difference Test with a 95\% family-wise confidence level along with two-way ANOVA using Tukey HSD post-hoc or using the car package for White's Corrected ANOVA for heteroscedasticity (72) along with a Tukey post-hoc test using glue (73) and userfriendlyscience $(74,75)$ packages in Rv3.5.0. Rhythmicity in gene expression profiles was assessed using JTK_CYCLE in R (76-78). Analysis using JTK_CYCLE was chosen as the program produces a robust reference curve that is suited for data with noise and a sampling frequency of four hours or less $(79,80)$. Settings included five biological replicates for each sample time point, sample interval of every four hours and to look for rhythms with a period range of eight to 24-hr. Relative quantities of gluR.2 and gluR8 were graphed in Rv3.5.0 $(61,81,82)$. 


\subsection{Results}

In the study transcriptional expression of two Exaiptasia pallida gluR genes was quantified to compare relative changes between the light cycles of $12 \mathrm{hr}$ light: $12 \mathrm{hr}$ dark (LD) and 12hr dark: $12 \mathrm{hr}$ dark (DD). Time and light treatment were analyzed for gluR.2 and gluR 8 gene expression, in addition to rhythm analysis for the $24 \mathrm{hr}$ sample period. The $24 \mathrm{hr}$ sample period compares LD to DD, the entrained LD cycle and constant darkness starting at 24-hrs within the DD cycle, to isolate endogenous versus dieldependent expression patterns.

\subsection{1 qPCR assays of Exaiptasia pallida gluR.2 under light treatments: The light}

treatment (LD vs. DD) did not significantly affect measured transcriptional expression of gluR.2 (Table 3.2; two-way ANOVA; $\mathrm{p}=0.1306$ ). Time was a factor in significantly differentiating the expression (Table 3.2; two-way ANOVA; $p=0.0007$ ). In LD, gluR.2 exhibited relatively high expression at $1400 \mathrm{hrs}$ with lowest expression at $1800 \mathrm{hrs}$, which increased again at $2200 \mathrm{hrs}$ (Fig. 3.4). In DD, transcriptional expression appears to vary significantly. Expression was elevated at $1000 \mathrm{hrs,} \mathrm{peaked} \mathrm{again} \mathrm{at} 2200 \mathrm{hrs}$ and had the lowest relative expression at 0200 hrs (Fig. 3.4). In both DD and LD, gluR.2 expression was relatively high followed by a decrease in relative expression quantity. The lowest point at LD 1800 hrs was significantly different from the peaks in expression at DD 1000 hrs and 2200 hrs (Fig. 3.4; two-way ANOVA; A p=0.0046, B p=0.0101). Within DD, 0200 hrs time point was significantly different to both 1000 hrs (Fig. 3.4, Table 3.2; two-way ANOVA; C p=0.0068) and 2200 hrs (Fig. 3.4, Table 3.2; two-way ANOVA; $\mathrm{D} p=0.0145)$. Between the diel cycles of LD and DD, the period changed from 
8-hr (Table 3.3; p-value $=0.0212$, JTK_CYCLE) to 16-hr $($ Table 3.3; p-value $=0.0039$, JTK_CYCLE) and the amplitude of expression increased (Table 3.3; LD 0.9167, DD 1.5526, JTK_CYCLE).

\subsection{2 qPCR assays of Exaiptasia pallida gluR8 under light treatments: Transcriptional} expression of gluR8 appeared relatively more consistent over time and with less spread for variance at each time point compared to gluR.2 particularly in LD (Fig. 3.5). Expression of gluR8 did not significantly differ as a function of the light treatment in statistical analysis (Fig. 3.5, Table 3.2; White's corrected two-way ANOVA; p=0.3331). However, the factor of time did significantly influence the expression patterns (Fig. 3.5, Table 3.2; White's corrected two-way ANOVA; $p=0.0031)$. In LD, time points of 1000 hrs and 1800 hrs differed significantly from DD 2200 hrs (Fig. 3.5, Table 3.2; corrected Tukey HSD; A p=0.0006, B p=0.0046). Expression values of gluR8 DD were fairly consistent with a single up-regulation peak at $2200 \mathrm{hrs}$. The timepoint of DD $2200 \mathrm{hrs}$ was significantly different compared to low expresion values measured at $0600 \mathrm{hrs}$ and 1000 hrs (Fig. 3.5, Table 3.2; corrected Tukey HSD; C p=0.0310, D p=0.0059). Periodicity changed from 8-hr in LD (Table 3.3.; p-value=0.0421, JTK_CYCLE) to 24-hr in DD (Table 3.3; p-value=0.0074, JTK_CYCLE). Unlike gluR.2, the amplitude decreased from LD to DD diurnal cycles (Table 3.3.; LD 0.7178, DD 0.5428, JTK_CYCLE). 


\subsection{Discussion}

From the innate function of iGluRs, the environment likely regulates gluR expression, whether external of the cnidarian host or via Symbiodiniaceae metabolism. Expression measurements imply gluRs were not predominantly acting in the role of 'pacemakers' for a circadian rhythm (83). Rather, the expression of gluRs followed an ultradian rhythm below 24-hr. The cycle measurements were produced from individuals with variation between biological replicates, opposed to aliasing from an observable consequence of the week or day sampled from potential lab conditions. Overall, the gluR.2 and gluR8 were observed to display statstically significant differences in rhythm periodicity between LD and DD.

3.6.1 Rhythm of expression in gluR.2: Expression of gluR.2 peaked at LD $1400 \mathrm{hrs}$ and again at LD 2200 hrs, with lowest expression at LD 1800 hrs. In comparison to DD, gluR.2 expression peaked at $1000 \mathrm{hrs}$ and $2200 \mathrm{hrs}$. While both gluRs increased in expression at $2200 \mathrm{hrs}$, in DD expression is up-regulated relative to the subsequent time point. The gluR.2 expression periodicity changes from LD 8-hr to DD 16-hr, which is consistent with a diel-controlled gene (DCG). The change in periodicity may signify constant LD oscillation is necessary to maintain expression (14) or an alternative biological rhythm. For instance, in marine intertidal areas, circatidal regulation is an influence of natural habit within a coastal environmental, in the intertidal zone (32). In this study, light or the affect of light on the biological factor of endosymbiosis with photosynthetic Symbiodiniaceae instigate variables for igluR.2 transcriptional response. 
3.6.2 Rhythm of expression in gluR8: Expression of gluR8 is up-regulated at $1800 \mathrm{hrs}$ in LD compared to 2200 hrs compared in DD (Fig. 3.4). Rhythm of gluR8 changes from an 8-hr period in LD to a circadian-like rhythm 24 -hr in DD, implying gluR8 is a clockcontrolled gene (CCG) as expression cycles are maintained under constant conditions. While CCG rhythm is not completely evident; Symbiodiniaceae are affected by diel cycles and in turn influence cnidarian gene expression, which is interpreted from the period shift in both genes. We postulate that dinoflagellate metabolic cycling affects the circadian nature of gluR8 while in constant LD cycling. Gene expression changes between LD vs DD are consistent with diel or light-dependent expression. In our experiment, nightly darkness occurred from $2000 \mathrm{hrs}$ to $0800 \mathrm{hrs,} \mathrm{placing} \mathrm{midnight} \mathrm{at}$ $0200 \mathrm{hrs}$ (Fig. 3.3). The expression of gluR8 peaked at $1800 \mathrm{hrs} \mathrm{LD} \mathrm{(Fig.} \mathrm{3.5).}$

3.6.3 Robustness of detecting host rhythms: Exaiptasia pallida gluR8 displays a 24-hr rhythm in DD, reminiscent of a circadian rhythm. While a 48-hour record is considered a standard, logistical and financial limitations presented a challenge in experimental design, which instigated the need for an alternative design (56). The study design provides a virgorously independent level of replication rather than a continuous series. In a continuous series experiment, an uncontrolled variable change over a single 48-hr experiment could have a very large impact on biasing the results. Through this method, we can observe for consistency in peak timing synchronized to the daily light cycle or within a 24-hour day. Failure to detect a rhythm may be due to aliasing, which is unlikely as anemones were entrained to the LD light cycle and experimental transitions to the dark remained the same for each replication. The detected cyclic patterns are more likely to be 
true temporal rhythms, whether endogenous or light-driven, over the five days of replication over a four week period. While the brevity of the study does make it difficult to calculate the length of the period with much accuracy, the objective of the study was to identify the sources of gene expression variation. Light and time were the factors measured, which very well correlate with metabolic diurnal cycling of Symbiodiniaceae. The shifts in rhythm are hypothesized to be a result of the change in glutamate levels under LD and the facultative symbiosis. If there is an influence of symbiosis on $E$. pallida gluR expression, then there would be a measurable difference between aposymbiotic and symbiotic anemones in diurnal cycles consistent with findings in Exaiptasia diaphana (32).

\subsubsection{Expression of iGluRs in Exaiptasia diaphana from transcriptomic data}

published by Sorek, et al. 2018: Physiological influences of cnidarian endosymbiosis have been studied across a range of symbiotic states including symbiotic reef-building corals, facultative symbiosis, and aposymbiotic cnidarians $(5,9,12,14,15)$. A recent study of aposymbiotic and symbiotic anemones in Exaiptasia diaphana (Rapp, 1829) detected biological rhythms for seven genes containing conserved domains associated to ionotropic receptor activity or extracellular-glutamate-gated ion channel activity exhibited (32). From the available metadata, we identified the Interpro conserved domains for iGluRs including ionotropic glutamate receptor L-glutamate and glycine binding domain (IPR019594, IPR001320), receptor ligand binding region (IPR001828, IPR028082), and metazoan ionotropic glutamate receptor (IPR001508) (Table 3.4) (32). The E. diaphana iGluRs displayed expression cycle patterns around a circatidal 12-hr 
period (Fig. 3.6). Four iGluRs, glutamate-ammonia ligase, and glutamate synthase have a 12-hr period (Table 3.4 and Fig. 3.6). One 12-hr period iGluRs (c160485) had no difference between the aposymbiotic and symbiotic states, which leads to a postulation that this particular iGluR transcription is not influenced by symbiosis. Three iGluRs had 24-hr periods (Table 3.4; Fig. 3.7). Two of these iGluRs were found in aposymbiotic anemones compared to one in symbiotic anemones. The differences between expression responses of iGluRs to symbiosis and to diurnal cycles lead to our hypothesis that genes identified as iGluRs have the capacity to function as a response to environmental signals from symbiotic metabolites. We speculate that cnidarian iGluRs can alter the potential to respond to specific ligands through acting in various pairs as co-receptors, while iGluRs with inverse expression patterns have different functions altogether. Another possibility for downstream physiological responses is multiple iGluRs must respond to two or more specific ligands, acting in concert to send a signal.

\subsubsection{Symbiodiniaceae and light influences on iGluR expression rhythms: Biological} rhythms are dynamic and complex, especially when attempting to identify the regulators or causation of gene expression cycles. From these findings and analysis the iGluR and related genes expression patterns from Sorek et al. (32), we hypothesize iGluR gene expression rhythms changed in response to diel stimulation. Cnidarian gene expression is altered by symbiosis, including metabolic changes influenced by light in symbiotic cnidarians $(4,31,32)$. Symbiodiniaceae are tightly associated with metabolic cycles in corals and anemones $(5,28,29)$. As light affects Symbiodiniacea metabolism, E. pallida gluR expression could change in response to the intercellular amount of glutamate 
available $(12,31,84)$. Conversely, gluR expression could decrease in preparation for the influx of glutamate with changing glutamate metabolism. In facultative symbiotic $E$. pallida, anemones with endosymbionts have documented up-regulated expression of glutamine synthetase and glutamate synthase, which is predicted to occur from host cnidarians altering nitrogen metabolism in accordance to Symbiodiniaceae (55). In this example, rather than acting in response to light entrainment, expression of glutamate transporters might respond to the diel-oriented glutamate concentration in cells to avoid overstimulation (85), which supports the hypotheses that E. pallida gluRs respond to environmental cues of pathogens and chemicals, or indirectly to diel rhythmicity through the symbiotic relationship with Symbiodiniaceae via light cues.

\subsection{Conclusion}

Diurnal expression analysis indicate that gluR.2 and gluR8 rhythms are influenced by time of day and effect of light on symbiosis. Along with symbiotic expression differences in E. diaphana, iGluR mRNA expression cycles are responsive to the light cycle. Expression of gluRs under LD is not consistent with predicted levels of levels of glutamate increasing at night from symbiosis. Internal molecular modulators of E. pallida may anticipate increases in glutamate synthesis when not under LD cycles and subsequent metabolic outputs from Symbiodiniaceae. These findings suggest that under normal operating conditions cnidarian, E. pallida and E. diaphana, iGluR transcription display innate ultradian biological rhythms, which we hypothesize is influenced by endosymbiosis with light-dependent Symbiodiniaceae . 
While for the most part, chemosensory receptors like iGluRs are known to respond to environmental cues in the form of chemical ligands, however, putative functions of cnidarian gluRs are unknown. The hypotheses developed from our findings of characterizing cnidarian iGluRs create a basis for functional studies. Measuring the rhythmicity of expression improves the knowledge base on iGluR presence within genomes and expression within cnidarians: organisms without a central nervous system to process neural signaling.

\subsection{Acknowledgements}

EGD and MRL conceived the experiment. EGD, with input from AMT, designed the experiment. EGD carried out the diurnal experiments and preserving samples. RNA extraction and cDNA synthesis were completed by EGD, BM and ML. EGD and BM prepared and ran qPCR assays. EGD analyzed expression data and ran statistical analyses. EGD, AMT, and MRL wrote the manuscript.

A special note of appreciation to Ann Tarrant (WHOI) for her invaluable recommendations and willingness to provide feedback on experimental design and manuscript. 


\subsection{Tables and Figures}

Table 3.1 Primer sequences for qPCR assay with sequence names and gene identity

\begin{tabular}{|c|c|c|l|}
\hline Gene & Sequence ID & Primer Name & \multicolumn{1}{|c|}{ Primer Sequence } \\
\hline \multirow{3}{*}{ gluR.2 } & 24462 & iGluR2f & ACGTGAGGAAGGAGAGATTGA \\
\cline { 2 - 4 } & 24462 & iGluR2r & AAGTGAAAAACCGGGCTGTG \\
\hline \multirow{3}{*}{ gluR8 } & 29007 & iGluR8f & TGCAGTTCTTGATGCCCTTTGA \\
\cline { 2 - 4 } & 29007 & iGluR8r & GACCAGAAGACGGCCTGATATAGA \\
\hline \multirow{3}{*}{ L10 } & 29093 & L10f & CTTGGACAGGAGCATCGATAATA \\
\cline { 2 - 4 } & 29093 & L10r & CCAGTGGGTTACGAAGACTTT \\
\hline \multirow{3}{*}{ L12 } & 8348 & L12f & TCACATGGTGTCAGATGAGTATG \\
\cline { 2 - 4 } & 8348 & L12r & CATACCAGTTTGGAGCCTATCA \\
\hline \multirow{3}{*}{ PABP } & 2230 & PABPf & AGAGAATTCCGCTTAGCAACTC \\
\cline { 2 - 4 } & 2230 & PABPr & GAAGCAAGCTGTCGCTTAGA \\
\hline
\end{tabular}




\begin{tabular}{|c|c|c|c|c|c|c|c|c|c|}
\hline 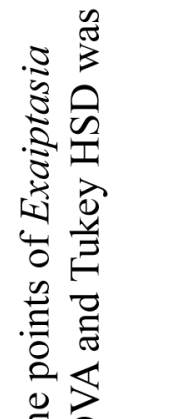 & 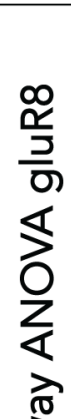 & $\begin{array}{l}\frac{0}{2} \\
\frac{\pi}{3} \\
\text { ف }\end{array}$ & $\begin{array}{l}\bar{m} \\
\text { m} \\
m \\
\stackrel{0}{0}\end{array}$ & $\begin{array}{l}\bar{m} \\
\stackrel{8}{8} \\
\dot{0}\end{array}$ & ¿ & 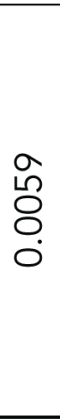 & $\begin{array}{l}\bar{m} \\
0 \\
0\end{array}$ & 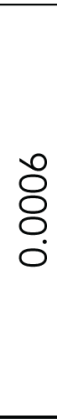 & 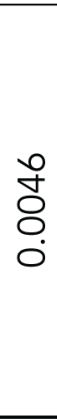 \\
\hline 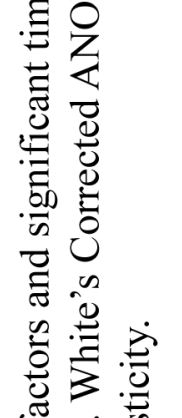 & 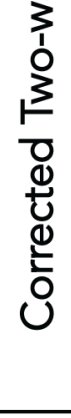 & 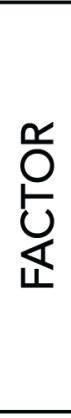 & $\begin{array}{l}\text { 贞 } \\
\underline{\underline{0}}\end{array}$ & $\underset{F}{\stackrel{\varpi}{\rightleftarrows}}$ & 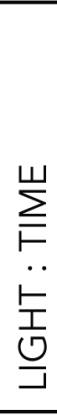 & 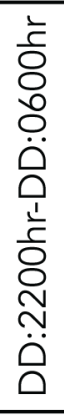 & 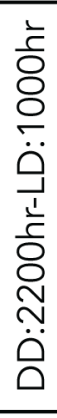 & 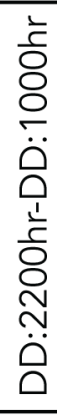 & 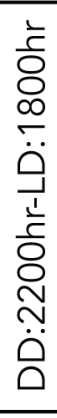 \\
\hline 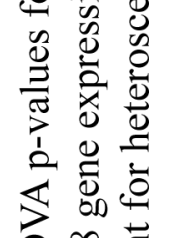 & 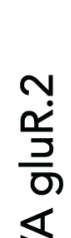 & $\begin{array}{l}\frac{0}{2} \\
\frac{\pi}{3} \\
\frac{1}{2}\end{array}$ & $\frac{\stackrel{\circ}{\circ}}{\stackrel{m}{\circ}}$ & $\begin{array}{l}\text { ○े } \\
8 \\
8\end{array}$ & $\begin{array}{l}\text { ○े } \\
\text { Oे } \\
\text { o. }\end{array}$ & $\begin{array}{l}\infty \\
8 \\
8 \\
\varnothing \\
0\end{array}$ & $\frac{\stackrel{1}{+}}{0}$ & $\begin{array}{l}\text { ¿ } \\
\text { ○ } \\
0\end{array}$ & $\begin{array}{l}\overline{0} \\
0 \\
0\end{array}$ \\
\hline 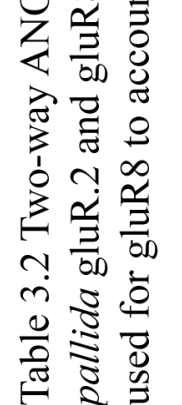 & 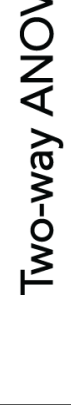 & 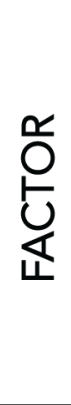 & $\begin{array}{l}\stackrel{\vdash}{I} \\
\stackrel{0}{\Xi}\end{array}$ & $\underset{F}{\stackrel{\rightleftarrows}{\Sigma}}$ & 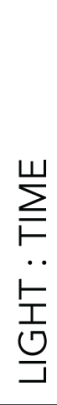 & 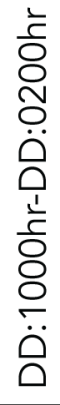 & 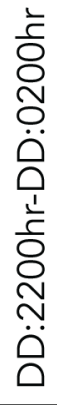 & 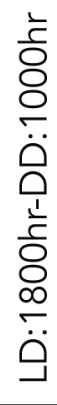 & 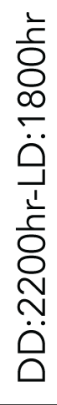 \\
\hline
\end{tabular}




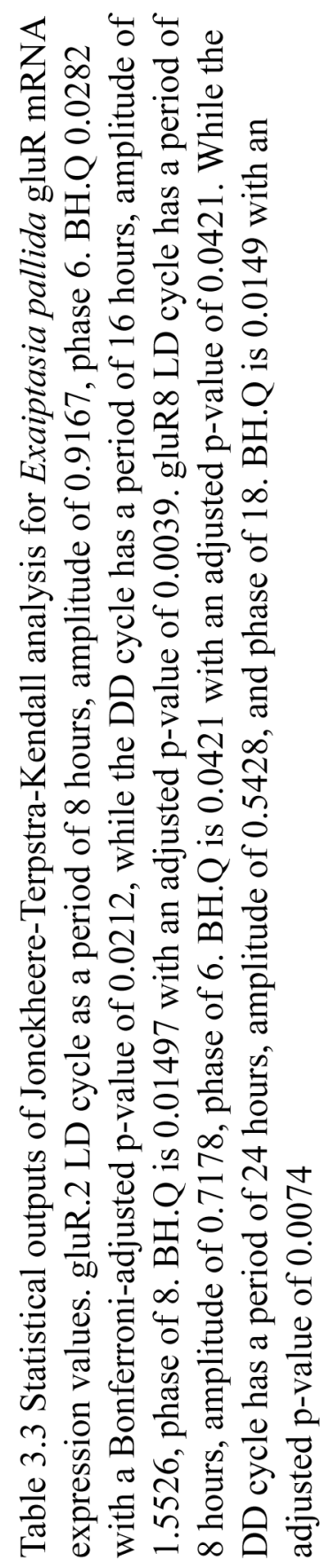

\begin{tabular}{|c|c|c|c|c|}
\hline$\frac{\frac{1}{0}}{\frac{2}{2}}$ & $\begin{array}{l}\hat{\sigma} \\
\frac{1}{0} \\
0\end{array}$ & 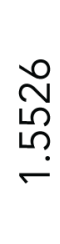 & $\frac{\infty}{\stackrel{\infty}{N}}$ & \begin{tabular}{l}
$\infty$ \\
\multirow{V}{*}{} \\
ம \\
0
\end{tabular} \\
\hline 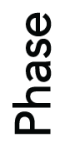 & ○ & $\infty$ & 0 & $\stackrel{\infty}{\sim}$ \\
\hline 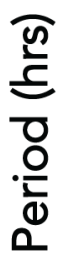 & $\infty$ & 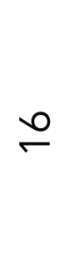 & $\infty$ & $\stackrel{む}{\sim}$ \\
\hline 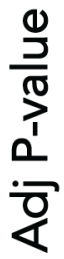 & $\begin{array}{l}\frac{N}{N} \\
\stackrel{\sigma}{0} \\
\stackrel{0}{0}\end{array}$ & 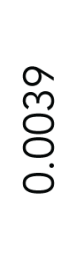 & 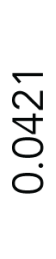 & $\begin{array}{l}\text { J } \\
\text { O̊ } \\
0\end{array}$ \\
\hline$\frac{O}{\frac{I}{\infty}}$ & 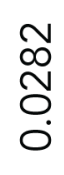 & $\begin{array}{l}a \\
\text { ป } \\
\vdots \\
0 \\
0\end{array}$ & 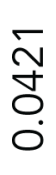 & $\begin{array}{l}\stackrel{\sigma}{\sigma} \\
\dot{0} \\
\dot{0}\end{array}$ \\
\hline$\frac{0}{U}$ & 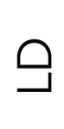 & ๑ & 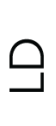 & $\bigcirc$ \\
\hline 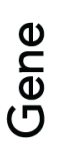 & & & & \\
\hline
\end{tabular}




\begin{tabular}{|c|c|c|c|c|c|c|c|c|c|}
\hline & $\begin{array}{l}\widetilde{O} \\
\frac{\mathrm{O}}{\mathbb{O}} \\
\stackrel{N}{V}\end{array}$ & $\begin{array}{l}\frac{\widehat{O}}{0} \\
\frac{\mathrm{O}}{\mathbb{N}} \\
\stackrel{\sim}{\sim}\end{array}$ & 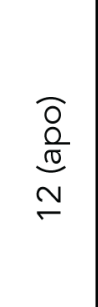 & 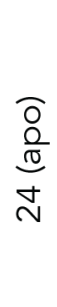 & 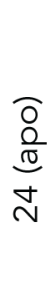 & 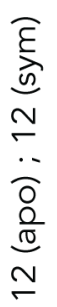 & 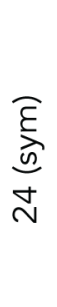 & 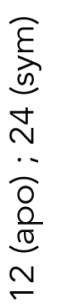 & 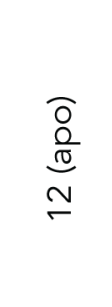 \\
\hline & 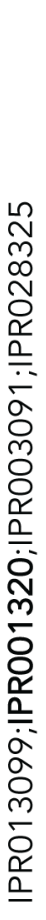 & 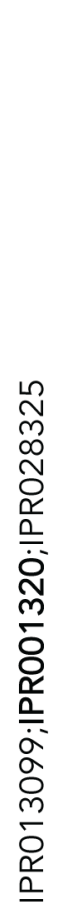 & 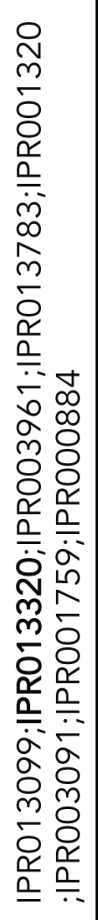 & 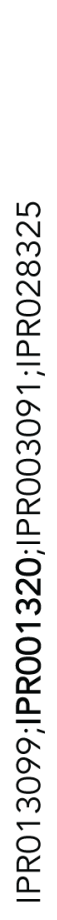 & 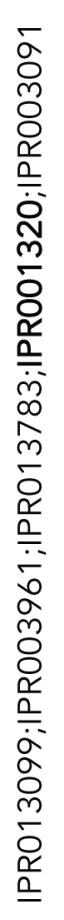 & 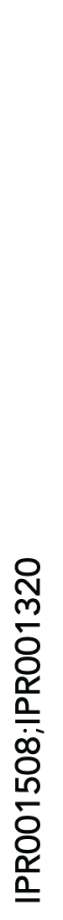 & 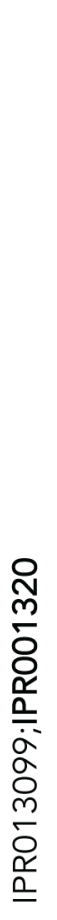 & 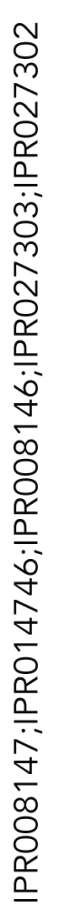 & 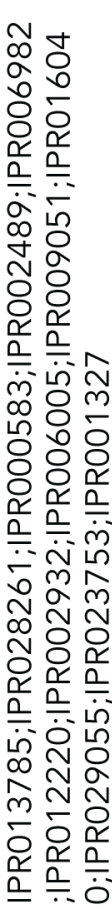 \\
\hline & $\frac{\frac{\alpha}{3}}{\underline{0}}$ & $\frac{\frac{\alpha}{3}}{\underline{0}}$ & $\frac{\frac{\alpha}{3}}{\frac{0}{0}}$ & $\frac{\underline{\alpha}}{\frac{\partial}{0}}$ & $\frac{\frac{\alpha}{J}}{\underline{0}}$ & $\frac{\frac{\alpha}{J}}{\underline{0}}$ & $\frac{\frac{\alpha}{3}}{\underline{0}}$ & 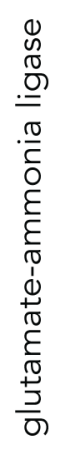 & 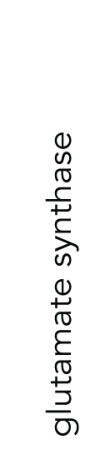 \\
\hline & 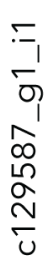 & $\begin{array}{l}\overline{-} \\
\bar{\sigma} \\
\bar{\sigma} \\
\bar{\infty} \\
\bar{\infty} \\
\tilde{n} \\
\overline{0}\end{array}$ & 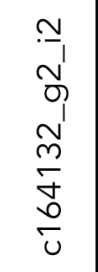 & $\begin{array}{l}\simeq 1 \\
\tilde{\sigma} \\
\tilde{\sigma} \\
\hat{N} \\
\tilde{0} \\
\tilde{0}\end{array}$ & 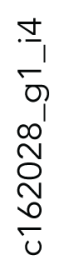 & $\begin{array}{l}= \\
-1 \\
0 \\
1 \\
0 \\
\infty \\
0 \\
0 \\
0 \\
\tilde{u}\end{array}$ & 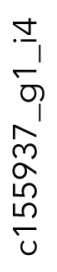 & $\begin{array}{l}= \\
- \\
\bar{\sigma} \\
1 \\
\infty \\
\infty \\
0 \\
\sigma \\
\bar{\sigma} \\
\bar{v}\end{array}$ & $\begin{array}{l}\cong \\
- \\
\sigma \\
\hat{N} \\
\tilde{n} \\
\frac{n}{0} \\
\tilde{U}\end{array}$ \\
\hline
\end{tabular}




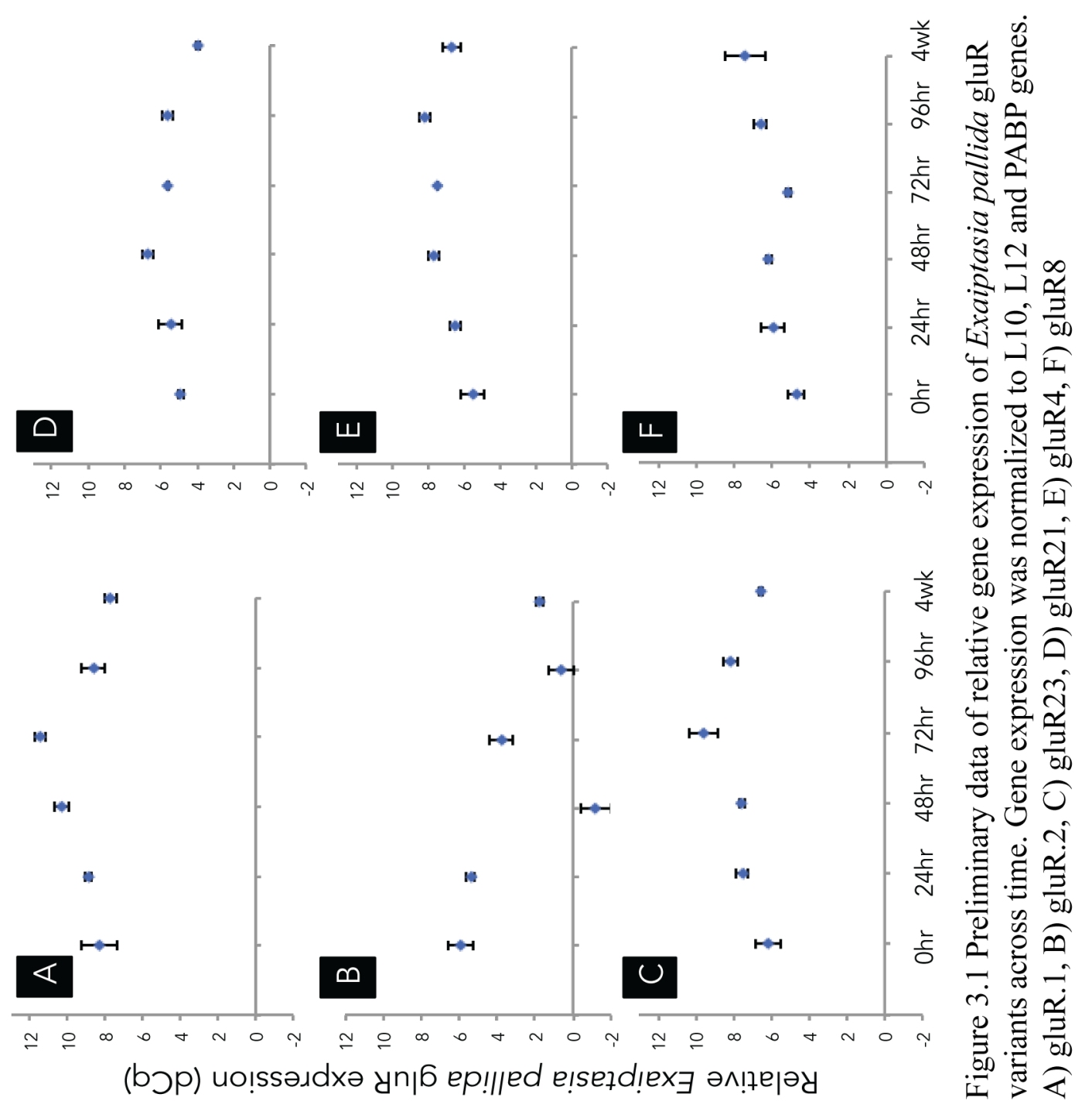




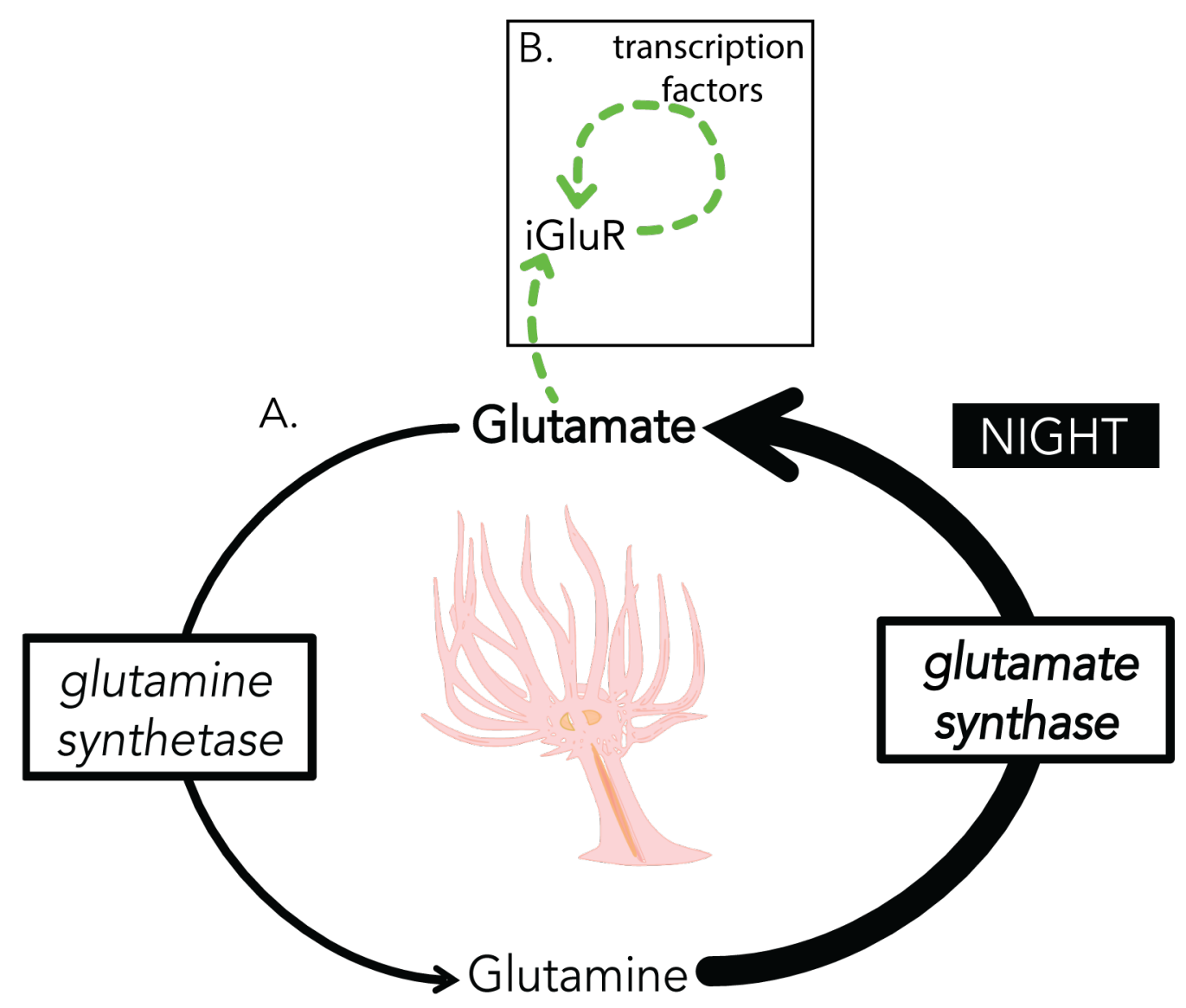

Figure 3.2 Representation of glutamate concentration cycling in cnidarians. A. levels of glutamate increase at night in consequence of the activation of glutamate synthase versus the shift to increasing levels of glutamine during the day with activation of glutamine synthetase in cnidarians. B. Putative response of iGluRs accompanied by the potential for downstream pathways affecting iGluR transcription from increased glutamate concentration. 


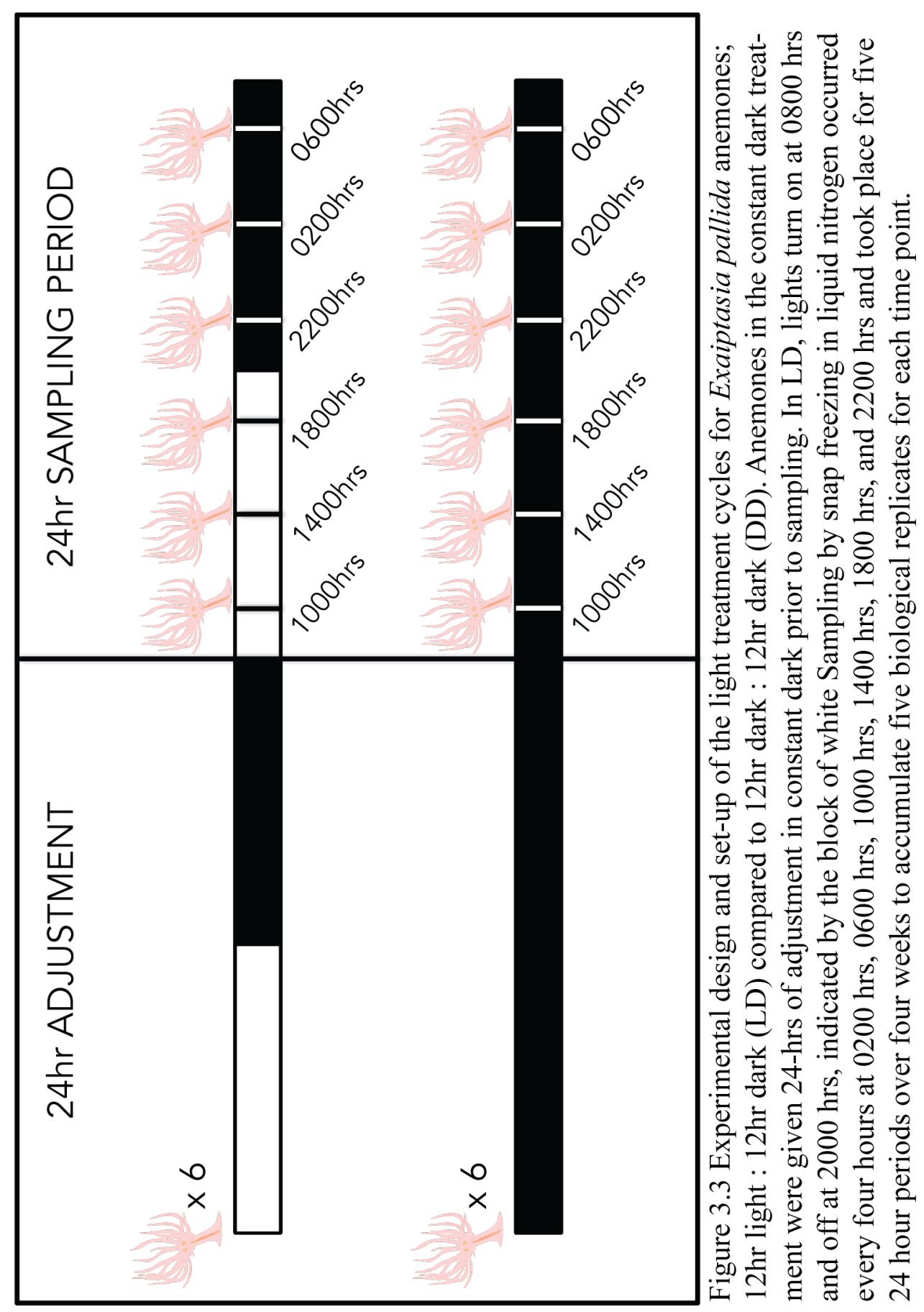




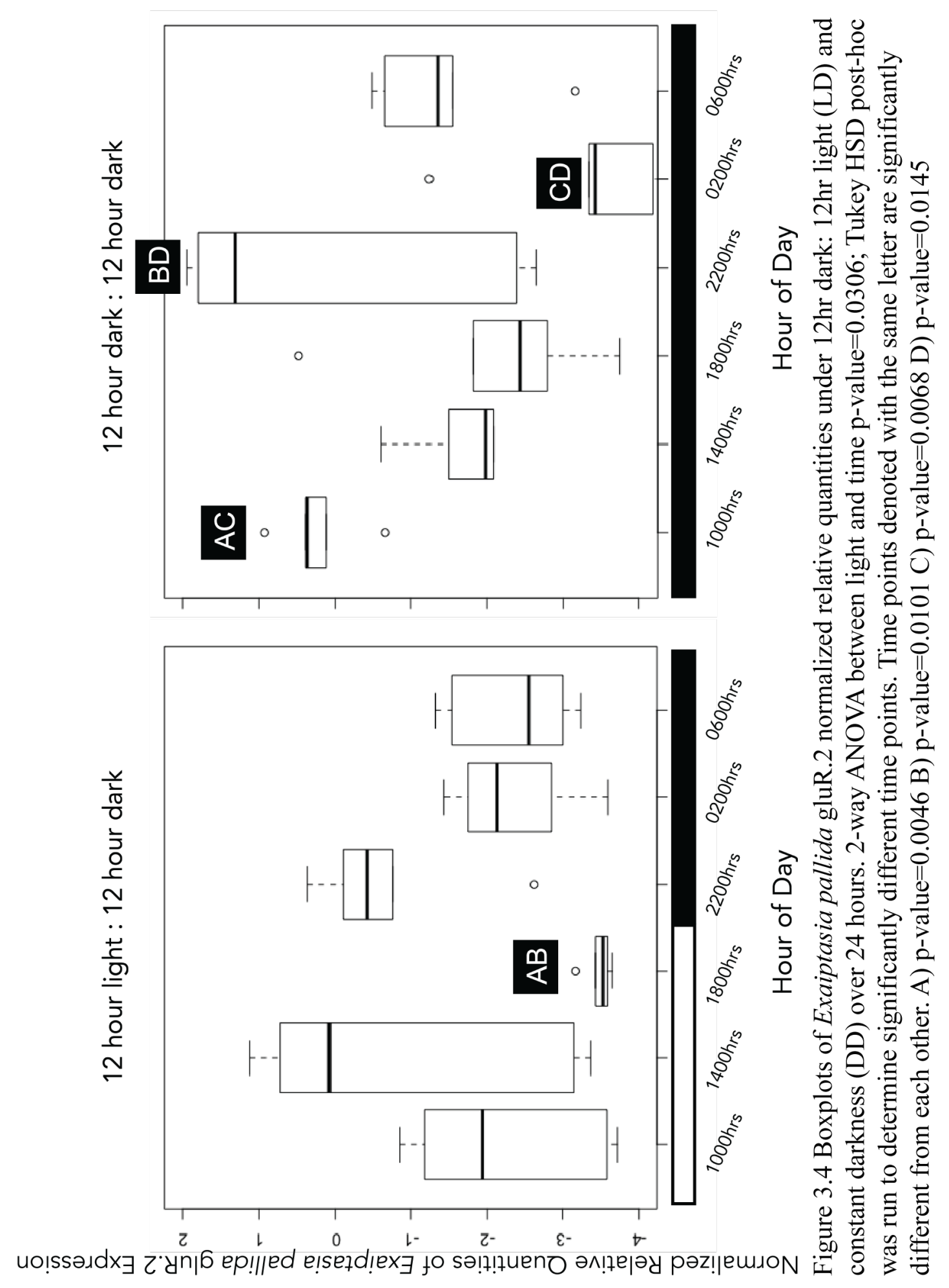




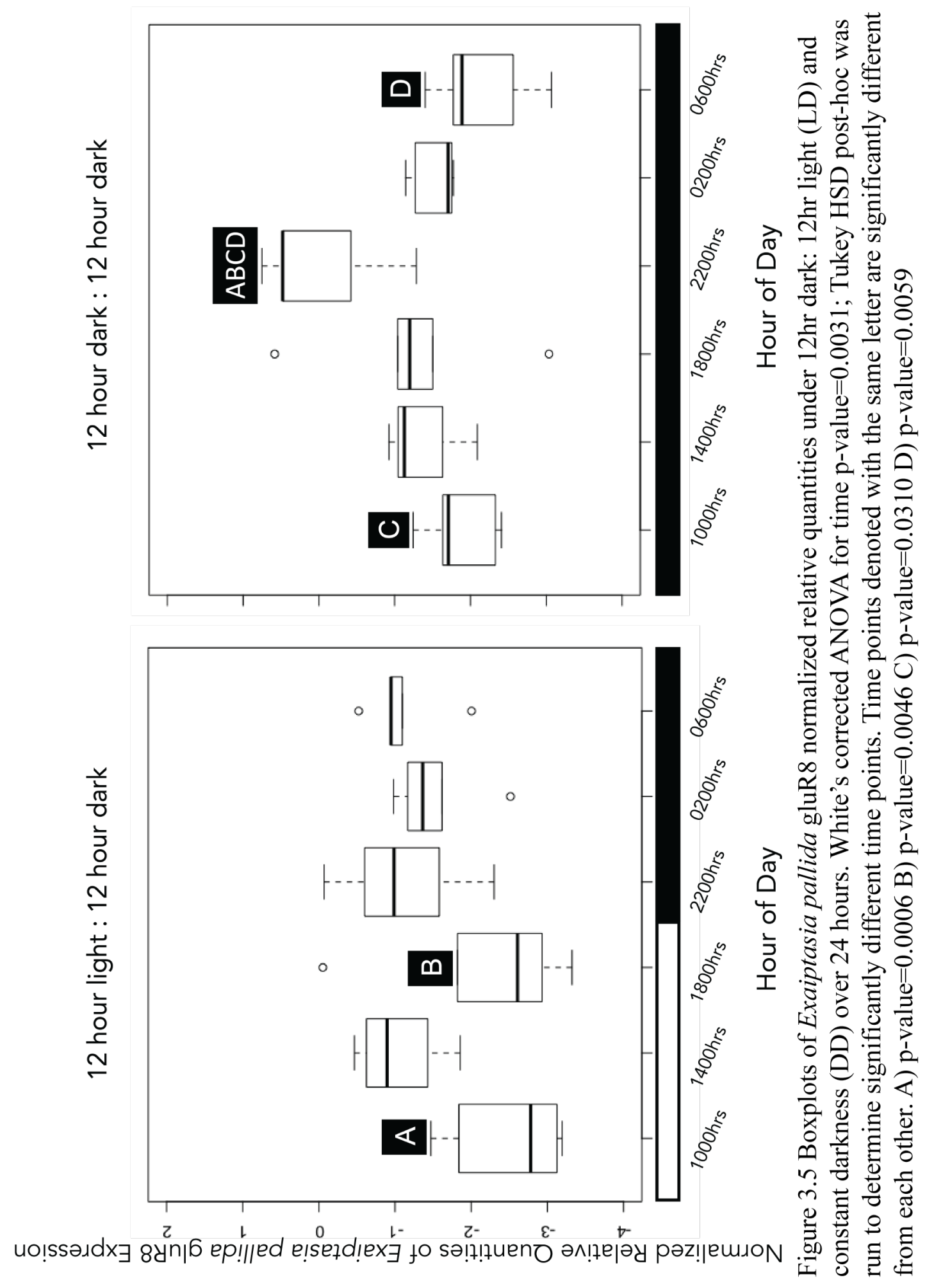



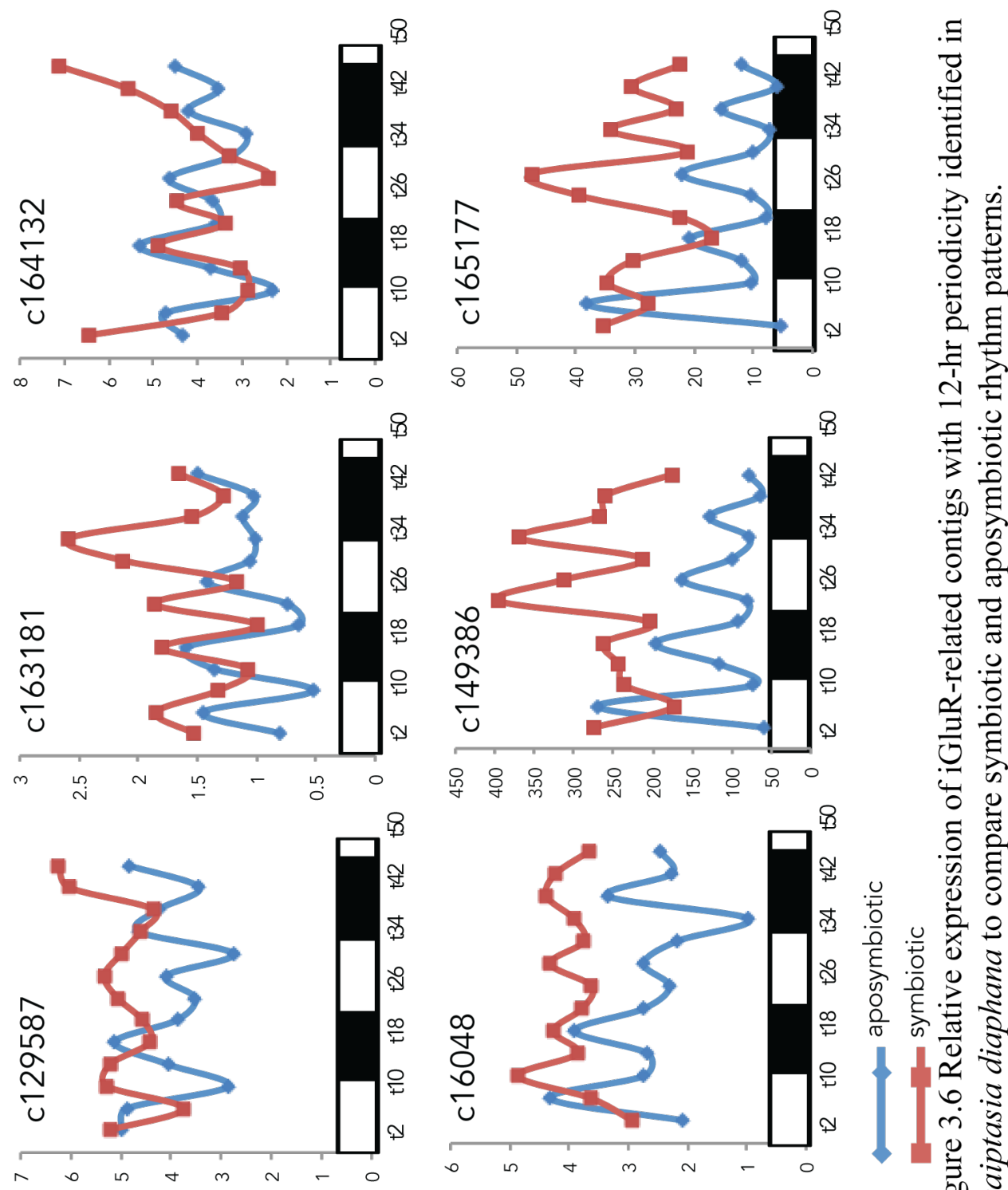

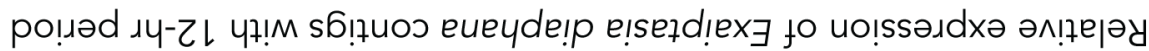




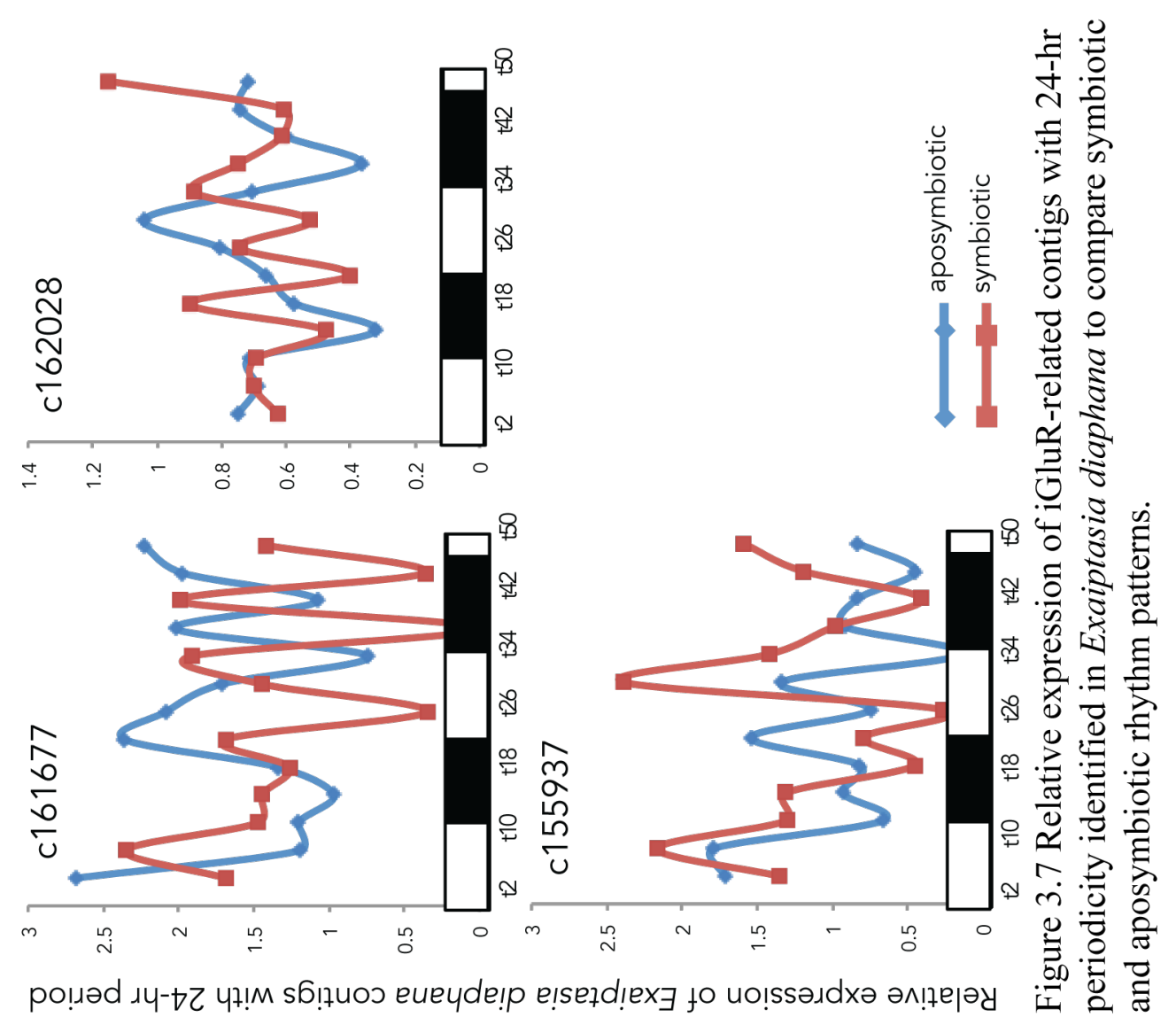




\subsection{References}

1. Butler AJ, Chesson PL. Ecology of sessile animals on sublittoral hard substrata: The need to measure variation. Aust J Ecol. 1990;15(4):521-31.

2. Seneca FO, Palumbi SR. The role of transcriptome resilience in resistance of corals to bleaching. Mol Ecol. 2015;

3. Putnam HM, Edmunds PJ. The physiological response of reef corals to diel fluctuations in seawater temperature. J Exp Mar Bio Ecol. 2011;396(2):216-23.

4. Sorek M, Levy O. Influence of the Quantity and Quality of Light on Photosynthetic Periodicity in Coral Endosymbiotic Algae. PLoS One. 2012;7(8):e43264.

5. Hemond EM, Vollmer S V. Diurnal and nocturnal transcriptomic variation in the Caribbean staghorn coral, Acropora cervicornis. Mol Ecol. 2015;24(17):4460-73.

6. Price NN, Martz TR, Brainard RE, Smith JE. Diel Variability in Seawater pH Relates to Calcification and Benthic Community Structure on Coral Reefs. PLoS One. 2012;7(8):1-9.

7. Oren M, Tarrant AM, Alon S, Simon-Blecher N, Elbaz I, Appelbaum L, et al. Profiling molecular and behavioral circadian rhythms in the non-symbiotic sea anemone Nematostella vectensis. Sci Rep. 2015 Jun 17;5:11418.

8. Sorek M, Díaz-Almeyda EM, Medina M, Levy O. Circadian clocks in symbiotic corals: The duet between Symbiodinium algae and their coral host. Mar Genomics. 2014;14:47-57.

9. Kaniewska P, Campbell PR, Fine M, Hoegh-Guldberg O. Phototropic growth in a reef flat acroporid branching coral species. J Exp Biol. 2009;212(5):662-7.

10. Raible F, Falciatore A. It's about time: Rhythms as a new dimension of molecular marine research. Mar Genomics. 2014;14(April):1-2.

11. Raible F, Falciatore A. Glossary Table. Nomenclature in the framework of (marine) rhythm biology. Mar Genomics. 2014;14(April):iii-iv.

12. Reitzel AM, Tarrant AM, Levy O. Circadian Clocks in the Cnidaria: Environmental Entrainment, Molecular Regulation, and Organismal Outputs. Integr Comp Biol. 2013;53(1):118-30.

13. Vize PD. Transcriptome Analysis of the Circadian Regulatory Network in the Coral Acropora millepora. Biol Bull. 2009;216:131-7. 
14. Reitzel AM, Behrendt L, Tarrant AM. Light Entrained Rhythmic Gene Expression in the Sea Anemone Nematostella vectensis: The Evolution of the Animal Circadian Clock. PLoS One. 2010;5(9):e12805.

15. Shoguchi E, Tanaka M, Shinzato C, Kawashima T, Satoh N. A genome-wide survey of photoreceptor and circadian genes in the coral, Acropora digitifera. Gene. 2013;515(2):426-31.

16. Levy O, Kaniewska P, Alon S, Eisenberg E, Karako-Lampert S, Bay LK, et al. Complex diel cycles of gene expression in coral-algal symbiosis. Science. 2011;331(6014):175.

17. Fitt WK. Cellular Growth of Host and Symbiont in a Symbiosis. Biol Bull. 2010;198(1):110-20.

18. Yellowlees D, Rees TA V, Leggat W. Metabolic interactions between algal symbionts and invertebrate hosts. Plant, Cell Environ. 2008;31:679-94.

19. Weis VM, Smith GJ, Muscatine L. A "CO2 supply" mechanism in zooxanthellate cnidarians: role of carbonic anhydrase. Mar Biol. 1989;100:195-202.

20. Sorek M, Schnytzer Y, Waldman Ben-Asher H, Chalifa Caspi V, Chen C-S, Miller DJ, et al. Setting the pace: host rhythmic behaviour and gene expression patterns in the facultatively symbiotic cnidarian Aiptasia are determined largely by Symbiodinium. Microbiome. 2018;6(83).

21. Vize PD, Hilton JD, Brady AK. Biological clock driven circadian transcription cycles in Acropora millepora. Proc 12th Int Coral Reef Symp Cairns, Aust. 2012;(July):9-13.

22. Hoadley KD, Vize PD, Pyott SJ. Current Understanding of the Circadian Clock Within Cnidaria. Cnidaria, Past, Present Futur. 2016;1-855.

23. Watanabe H, Fujisawa T, Holstein TW. Cnidarians and the evolutionary origin of the nervous system. Dev Growth Differ. 2009;51:167-83.

24. Liebeskind BJ, Hofmann HA, Hillis DM, Zakon HH. Evolution of Animal Neural Systems. Annu Rev Ecol Evol Syst. 2017;48:377-98.

25. Willard SS, Koochekpour S. Glutamate, Glutamate Receptors, and Downstream Signaling Pathways. Int J Biol Sci. 2013;9:949-59. 
26. Koo JCP, Hampson DR. Phylogenic and Evolutionary Analysis of Glutamate Receptors Based on Extant Invertebrate Genes. J Undergrad Life Sci. 2010;4(1):42-9.

27. Rytz R, Croset V, Benton R. Ionotropic Receptors (IRs): Chemosensory ionotropic glutamate receptors in Drosophila and beyond. Insect Biochem Mol Biol. 2013;43:888-97.

28. Bigge CF. lonotropic glutamate receptors. Curr Opin Chem Biol. 1999;3:441-7.

29. Benton R. Multigene Family Evolution: Perspectives from Insect Chemoreceptors. Trends Ecol Evol. 2015;30(10):590-600.

30. Eyun S-I, Soh HY, Posavi M, Munro JB, Hughes DST, Murali SC, et al. Evolutionary History of Chemosensory-Related Gene Families across the Arthropoda. Mol Biol Evol. 2017;34(8):1838-62.

31. Hayashi T. Evolutionarily conserved palmitoylation-dependent regulation of ionotropic glutamate receptors in vertebrates. Neurotransmitter. 2014 Jan;1(e388).

32. Greer JB, Khuri S, Fieber LA. Phylogenetic analysis of ionotropic L- glutamate receptor genes in the Bilateria, with special notes on Aplysia californica. BMC Evol Biol. 2017;17(11).

33. Collingridge GL, Lodge D, Mayer M, Turrigiano G, Frenguelli BG. Ionotropic glutamate receptors: Still exciting after all these years. Neuropharmacology. 2017;112:1-3.

34. Varoqueaux F, Fasshauer D. Getting Nervous: An Evolutionary Overhaul for Communication. Annu Rev Genet. 2017;51:455-76.

35. Benton R, Vannice KS, Gomez-Diaz C, Vosshall LB. Variant Ionotropic Glutamate Receptors as Chemosensory Receptors in Drosophila. Cell. 2009;136(149-162).

36. Watanabe H. Back Through Time: How Cnidarians and Basal Metazoans Shed Light on Ancient Nervous Systems. In: Shigeno S, Murakami Y, Nomura T, editors. Diversity and Commonality in Animals: Brain Evolution by Design From Neural Origin to Cognitive Architecture. Japan: Springer, Diversity and Commonality in Animals; 2017. p. 45-75.

37. Croset V, Rytz R, Cummins SF, Budd A, Brawand D, Kaessmann H, et al. Ancient protostome origin of chemosensory ionotropic glutamate receptors and the evolution of insect taste and olfaction. PLoS Genet. 2010;6(8):e1001064. 
38. Abuin L, Bargeton B, Ulbrich MH, Isacoff EY, Kellenberger S, Benton R. Functional Architecture of Olfactory Ionotropic Glutamate Receptors. Neuron. 2011;69:44-60.

39. Chen C, Buhl E, Xu M, Croset V, Rees JS, Lilley KS, et al. Drosophila Ionotropic Receptor 25a mediates circadian clock resetting by temperature. Nature. 2015;527:516-20.

40. Van Giesen L, Garrity PA, Grunwald-Kadow I. More than meets the IR: the expanding roles of variant Ionotropic Glutamate Receptors in sensing odor, taste, temperature and moisture. F1000Research. 2017;6:1753.

41. Golombek DA, Rosenstein RE. Physiology of Circadian Entrainment. Physiol Rev. 2010;90:1063-102.

42. Smith OP, Marinov AD, Chan KM, Ferrier MD. Cloning and sequencing of cDNA encoding glutamine synthetase from the sea anemone Aiptasia pallida. Hydrobiologia. 2004;530-531:267-72.

43. Lehnert EM, Mouchka ME, Burriesci MS, Gallo ND, Schwarz JA, Pringle JR. Extensive Differences in Gene Expression between Symbiotic and Aposymbiotic Cnidarians. G3 Genes Genomes Genet. 2014;4(2):277-95.

44. Hughes ME, Abruzzi KC, Allada R, Anafi R, Arpat AB, Asher G, et al. Guidelines for Genome-Scale Analysis of Biological Rhythms. J Biol Rhythms. 2017;32(5):380-93.

45. TATAA Genomics. User Manual TATAA Interplate Calibrator SYBR protocol. 2012;(August):1-16.

46. Ruijter JM, Ruiz Villalba A, Hellemans J, Untergasser A, van den Hoff MJB. Removal of between-run variation in a multi-plate qPCR experiment. Biomol Detect Quantif. 2015;5:10-4.

47. Hellemans J, Mortier G, Paepe A, Speleman F, Vandesompele J. qBase relative quantification framework and software for management and automated analysis of real-time quantitative PCR data. Genome Biol. 2007;8(2):R19.

48. Vandesompele J, De Preter K, Pattyn ilip, Poppe B, Van Roy N, De Paepe A, et al. Accurate normalization of real-time quantitative RT-PCR data by geometric averaging of multiple internal control genes. Genome Biol. 2002;3(7):0034.1-12.

49. Ling D, Salvaterra PM. Robust RT-qPCR data normalization: Validation and selection of internal reference genes during post-experimental data analysis. PLoS One. 2011;6(3). 
50. Kozera B, Rapacz M. Reference genes in real-time PCR. Plant Genet. 2013;54:391-406.

51. Rosic NN, Pernice M. Validation of Housekeeping Genes for Gene Expression Studies in Symbiodinium Exposed to Thermal and Light Stress. Mar Biotechnol. 2011;13:355-65.

52. Rodriguez-Lanetty M, Phillips WS, Dove S, Hoegh-Guldberg O, Weis VM. Analytical approach for selecting normalizing genes from a cDNA microarray platform to be used in q-RT-PCR assays : A cnidarian case study. J Biochem Biophys Methods. 2008;70(6):985-91.

53. Pfaffl MW. Quantification strategies in real-time PCR. A-Z Quant PCR. 2004;87112.

54. Pabinger S, Rödiger S, Kriegner A, Vierlinger K, Weinhäusel A. A survey of tools for the analysis of quantitative PCR (qPCR) data. Biomol Detect Quantif.

2014;1:23-33.

55. Boyer TC, Hanson T, Singer RS. Estimation of Low Quantity Genes: A Hierarchical Model for Analyzing Censored Quantitative Real-Time PCR Data Estimation of Low Quantity Genes: A Hierarchical Model for Analyzing Censored Quantitative Real-Time PCR. PLoS One. 2013;8(5):e64900.

56. Peña EA, Slate EH. Global Validation of Linear Model Assumptions. J Am Stat Assoc. 2006;101(473):341-54.

57. Royston JP. An Extension of Shapiro and Wilk's W Test for Normality to Large Samples. Source J R Stat Soc Ser C (Applied Stat. 1982;31(2):115-24.

58. Royston JP. Algorithm AS 181: The W Test for Normality. Source J R Stat Soc Ser C (Applied Stat. 1982;31(2):176-80.

59. R Core Team. R: A Language and Environment for Statistical Computing. Vienna, Austria: R Foundation for Statistical Computing; 2014.

60. Fox J, Weisberg S. An R companion to applied regression. 2nd ed. Thousand Oaks, Calif.: SAGE Publications; 2011.

61. Hester J. glue Interpreted String Literals for R: An implementation of Interpreted String Literals (f-strings) for R.

62. Peters G. userfriendlyscience: Quantitative analysis made accessible. https://userfriendlyscience.com.; 2018. 
63. Peters GY. Diamond plots: a tutorial to introduce a visualisation tool that facilitates interpretation and comparison of multiple sample estimates while respecting their inaccuracy. PsyArXiv. 2017;

64. Hughes ME, Hogenesch JB, Kornacker K. JTK_CYCLE: an efficient nonparametric algorithm for detecting rhythmic components in genome-scale datasets. J Biol Rhythm. 2011;25(5):372-80.

65. Miyazaki M, Schroder E, Edelmann SE, Hughes ME, Balke CW, Esser KA. AgeAssociated Disruption of Molecular Clock Expression in Skeletal Muscle of the Spontaneously Hypertensive Rat. PLoS One. 2011;6(11):1-11.

66. Ren Y, Hong CI, Lim S, Song S. Finding Clocks in Genes: A Bayesian Approach to Estimate Periodicity. Biomed Res Int. 2016;3017475.

67. Hutchison AL, Maienschein-Cline M, Chiang AH, Tabei SMA, Gudjonson H, Bahroos N, et al. Improved Statistical Methods Enable Greater Sensitivity in Rhythm Detection for Genome-Wide Data. PLoS Comput Biol. 2015;11(3):1-29.

68. Wu G, Anafi RC, Hughes ME, Kornacker K, Hogenesch JB. Systems biology MetaCycle : an integrated $\mathrm{R}$ package to evaluate periodicity in large scale data. Bioinformatics. 2016;32(July):3351-3.

69. Krzywinski M, Altman N. Visualizing samples with box plots. Nat Methods. 2014;11(2).

70. Bustin SA, Benes V, Garson J, Hellemans J, Huggett J, Kubista M, et al. The need for transparency and good practices in the qPCR literature. Nat Methods. 2013;10(11):1063-7.

71. Rusak B, Zucker I. Neural Regulation of Circadian Rhythms. Physiol Rev. 1979;59(3):449-526.

72. Venn AA, Loram JE, Douglas AE. Photosynthetic symbioses in animals. J Exp Bot. 2008;59(5):1069-80.

73. Oakley CA, Ameismeier MF, Peng L, Weis VM, Grossman AR, Davy SK. Symbiosis induces widespread changes in the proteome of the model cnidarian Aiptasia. Cell Microbiol. 2016;18(7):1009-23.

74. Rosic N, Yew E, Ling S, Chan C-KK, Lee HC, Kaniewska P, et al. Unfolding the secrets of coral-algal symbiosis. ISME J. 2015;9182(10):844-56. 
75. Weis VM, Levine RP, Station HM, Grove P. Differential protein profiles reflect the different lifestyles of symbiotic and aposymbiotic Anthopleura elegantissima, a sea anemone from temperate waters. J Exp Biol. 1996;199:883-92.

76. Chi-Castañeda D, Ortega A. Circadian Regulation of Glutamate Transporters. Front Endocrinol (Lausanne). 2018;9(340). 


\section{CHAPTER IV. LOCALIZATION OF IONOTROPIC GLUTAMATE RECEPTORS \\ WITHIN THE CNIDARIAN EXAIPTASIA PALLIDA VIA IN SITU HYBRIDIZATION}

\subsection{Abstract}

Ionotropic glutamate receptors (iGluRs) provide chemical sensory signals to various metazoans, including a recently uncovered expansion of homologs within Exaiptasia pallida. While the response of E. pallida iGluRs is correlated to bacteria-specific signaling and environmental processing, it is important to now understand where these genes are expressed. Cnidarian iGluRs are proposed to work by detecting external signals from bacteria, potential extracellular ligands, and external environment cues. Defensive or offensive spirocyst discharging may involve iGluRs via glutamate as a neurotransmitter within the dispersed sensory system that lies within the epidermis. Through developing mRNA in situ hybridization techniques for the sea anemone $E$. pallida, iGluR gene expression was located primarily in tentacles, which contain high densities of nematocytes and spirocytes, in addition to the polyp column for select iGluRs. From these findings, gene expression of iGluRs is closely tied to the epiderm that contains sensory cells and along the nerve net. In addition to cnidocytic protective mechanisms within the anemone E. pallida, iGluRs are predicted to be involved in the perpetuation of neural signals throughout the polyp column. Exaiptasia pallida iGluR RNA expression was localized to the epidermis near sensory cells, congregated in the tentacles in greater densities relative to the polyp column of anemones, which is consistent with the chemosensory nature of this family of genes. 


\subsection{Keywords}

Exaiptasia pallida, in situ localization, chemosensory, iGluR, Cnidaria

\subsection{Introduction}

Cnidarians, like many marine invertebrates, utilize various receptor pathways within their innate immune systems to recognize non-self and regulate interactions with organisms, such as microbes in the water column and symbiotic dinoflagellates (Symbiodiniaceae) (1-7). The phylum Cnidaria includes sessile organisms, corals and anemones, which introduces an obstacle to overcome with physiological responses $(8,9)$. The first barrier in organismal defense includes recognition via the immune system. Immune-associated cells that identify non-self components i.e., immunocytes, are inhibited by immunosuppression chemicals, which prevent fusion and rejection during allogeneic experiments and in turn suppress the immune response $(10,11)$. Immune gene expression in cnidarians has been associated with the gastroderm $(2,8)$, which is consistent with recognition cells located in gastrodermal tissues that are often exposed to external organisms and chemicals taken in through the pharynx into the gastric cavity (Fig. 4.1A). To perpetuate communication and elicit defense responses from immune signals of changing environments, cnidarians require cells that function as receptors to recognize environmental factors.

Recognition receptors that distinguish infections may function differently within a dispersed nervous system in contrast to derived metazoans with bilateral body plans and centralized neural networks (12-14). These receptors may have multiple functional roles or diverged in evolution to serve as a connection between nervous systems and immune 
recognition. A family of chemosensory receptors, ionotropic glutamate receptors (iGluRs) may have likely evolved in cnidarians to connect chemosensation and neural signaling with innate immunity (Ch. 2). The iGluR receptor family is responsible for perceiving signals, particularly from environmental chemicals within many invertebrates from insects to mollusks (15-17). While cnidarian iGluRs have been implicated in a chemosensory or immune response to bacteria (Ch. 2), the location of where iGluRs are expression is unknown within cnidarians $(18,19)$. To better understand potential functions of cnidarian iGluRs, mapping the localization of iGluRs within the cnidarian nervous system is of utmost importance.

The cnidarian nervous system for anemones is a nerve net of interconnected nerve fibers, neurons and neurites that form synaptic contacts, dispersed between epithelial cells of the epiderm (20). The nervous system consists of sensory cells that receive information, ganglion cells that process information, from neuroglandular synapses within the pharynx to mechanically responsive muscle cells located at the base of the epidermal epithelium $(20,21)$. Receptors send signals into cells via neural networks, beginning with apical cilium sensory cells, which lead to one- or two-way interneuronal, neuromuscular, and uniquely cnidarian neuro-nematocyte synapses (22-24). Cnidarian tentacles contain stinging cells: nematocytes and spirocytes, that have microscopic harpoons called nematocysts and spirocysts, respectively (Fig. 4.1; 16-19). Discharge of nematocysts was thought to be under local control by chemical and mechanical triggers, including neural control of spirocyst discharge $(27,28)$; known as the independent effector hypothesis (without neural involvement), a three-cell pathway with a ganglion 
cell integrated motor functions and sensory cells triggered spirocytes by vibratory and chemical stimuli from prey (25). However, synaptic modulation is involved in neural control of spirocyst discharge and more than one neurotransmitter can control spirocyst discharge with changes to mechanistic physiology $(21,24,29)$. Sea anemones contain more ganglion than sensory types of neurons, which include dissociated neural cells within tentacles (22). The proximity of iGluRs to the nerve net, related ganglion and sensory neurons is unknown.

From our knowledge of iGluR function, putative specific ligands from phylogenetics (Ch. 2), and the involvement of glutamate in discharging spirocysts and nematocysts $(25,27,29)$, there is a plausible connection of iGluRs and defense in cnidarians within the sea anemone Exaiptasia pallida. These connections have created speculation as to where iGluRs are expressed and involvement in the neurosensory network. By localizing expression through chromogenic mRNA in situ hybridization is the first step in understanding the function of iGluRs in E. pallida.

\subsection{Methods}

4.4.1 Animal maintenance: Clonal symbiotic E. pallida anemones (CC7) were maintained in artificial seawater at approximately $27^{\circ} \mathrm{C}$. Populations were kept on a day/night cycle of 12 hours light: 12 hours dark with 30 to $60 \mu \mathrm{mol}$ photons $\mathrm{m}^{-2} \mathrm{~s}^{-1}$ of light intensity and fed freshly hatched brine shrimp Artemia nauplii twice a week. Exaiptasia pallida anemones fixed for in situ hybridization were approximately $300 \mu \mathrm{m}$ 
in diameter and $750 \mu \mathrm{m}$ in length, while anemones used for RNA extraction were larger at 2-3 $\mathrm{mm}$ in diameter and 6-8 $\mathrm{mm}$ in length.

4.4.2 RNA extraction and cDNA synthesis: The RNeasy mini kit (Qiagen, Valencia, CA) and Monarch Total RNA Miniprep Kit (New England Biolabs, Ipswich, MA) were used to extract RNA. The samples used on-column DNase treatment (New England Biolabs, Ipswich, MA) and 2 additional $80 \%$ EtOH washes. We eluted RNA in a final volume of 30 or $50 \mu \mathrm{L}$, respective of kit, with nuclease-free water. Quantification of extracted RNA was conducted using a NanoDrop 2000 (ThermoFisher, Carlsbad, CA) and RNA integrity assessed visually on a $1 \%$ TAE bleach gel (30). Samples with a spectrophotometric 260/230 ratio of less than 1.5 underwent additional cleanup using $10 \% 3 \mathrm{M}$ sodium acetate ( $\mathrm{pH}$ 5.2), RNA-grade glycogen at a final concentration of $0.05-$ $1.0 \mu \mathrm{g} / \mathrm{mL}$, and 2.5 volumes of $100 \% \mathrm{ETOH}$ to the sample. Following precipitation overnight at $-80{ }^{\circ} \mathrm{C}$, samples were centrifuged for $20 \mathrm{~min}$ at $9500 \mathrm{rpm}\left(4^{\circ} \mathrm{C}\right)$ to pellet RNA. The pellet was washed with $250 \mu \mathrm{L}$ of $70 \% \mathrm{ETOH}$, centrifuged for $5 \mathrm{~min}$ at the same conditions listed above, and dried before re-suspending in $20-40 \mu \mathrm{L}$ of nucleasefree water. cDNA synthesis was performed with Superscript III first strand synthesis system using Oligo dT primer (Life Technologies, Eugene, OR) and 200-400 ng of RNA.

4.4.3 Exaiptasia pallida RNA probe design: Full-length PCR primers between 18 and $25 \mathrm{bp}$ in length were designed with an optimal $\mathrm{Tm}$ of $60^{\circ} \mathrm{C}$ for $E$. pallida iGluR genes (gluR) using PrimerQuest (IDT, Coralville, IA). For PCR analysis, each targeted gene of interest was prepared with a 31 of $\mathrm{H}_{2} \mathrm{O}, 5 \mu \mathrm{L}$ of $10 \mathrm{x}$ Buffer for KOD polymerase, $5 \mu \mathrm{L}$ 
dNTPs, $3 \mu \mathrm{L} \mathrm{MgSO}_{4}, 1.5 \mu \mathrm{L}$ of each primer, $2 \mu \mathrm{L}$ cDNA from E. pallida, $1 \mu \mathrm{L}$ KOD polymerase (Billerica MA). Reactions were run on a thermocycle of initial polymerase activation of 2 min at $95^{\circ} \mathrm{C}$, followed by 39 cycles of 20 s denaturation at $95^{\circ} \mathrm{C}$, annealing for $30 \mathrm{~s}$ at a gradient from $52{ }^{\circ} \mathrm{C}$ to $59{ }^{\circ} \mathrm{C}$, followed by extension for $60 \mathrm{~s}$ at 70 ${ }^{\circ} \mathrm{C}$. Products were checked for size on a gel using $10 \mu \mathrm{L}$ of PCR product. The remaining $40 \mu \mathrm{L}$ of PCR product was processed through the NEB PCR and DNA Cleanup (New England BioLabs, Ipswich MA). For amplicon isolation, the low concentration reactions were pooled and bands extracted from agarose gels. Concentrations were determined using a NanoDrop 2000 (ThermoFisher, Carlsbad, CA).

The NEB PCR Cloning Kit was used to ligate and transform PCR product into competent E. coli cells following kit protocol (New England BioLabs, Ipswich MA). Cloned products (Protocol Exemption IBC-17-017) were isolated using Plasmid MiniPrep kit from NEB (New England BioLabs, Ipswich MA) and sequenced using primers from the kit. Cloned genes are listed in Table 4.1. Once gluR sequences were verified, PCR was used to re-amplify gluRs with additional pGEMHE overhangs (Table 4.2) and generate a linear for pGEMHE vector with the selected insertion site. Correct band size of product was gel extracted and purified. The gluR inserts and pGEMHE were assembled using the NEB HiFi Assembly kit (New England BioLabs, Ipswich MA) and then transformed into competent cells. Transformations were screened using ampicillin resistance, colony PCR, and sequencing. Plasmids were extracted using Plasmid MidiPrep kit from NEB (New England BioLabs, Ipswich MA). Plasmids were then completely digested as prescribed using SmaI or Sbfl restriction digest enzymes (New 
England BioLabs, Ipswich MA). Probes were synthesized via in vitro transcription with the Digoxigenin (DIG) RNA Labeling Kit using Sp6 polymerase for the antisense probes (digested with SmaI) or T7 polymerase for sense probes (digested with SbfI) (Roche Life Science, Indianapolis IN).

4.4.4 Chromogenic RNA in situ hydridization: In situ hybridization was carried out on Exaiptasia pallida anemones following an adapted protocol from Nematostella vectensis $(31,32)$ and Cassiopea sp. following suggestions from Leslie Babonis and Bailey Steinworth (Martindale Lab, Whitney Labs, University of Florida) completing between four to ten biological replicates per gene. Anemones were immobilized in $7 \% \mathrm{MgCl}_{2}$ in filtered seawater, then fixed in ice-cold $4 \%$ paraformaldehyde with $0.2 \%$ glutaraldehyde for $1 \mathrm{~min} 30 \mathrm{sec}$, followed by $4 \%$ paraformaldehyde in phosphate-buffered saline with $1 \%$ Tween $(\mathrm{PTw})$ at $\mathrm{pH} 8.8$ for $1 \mathrm{hr}$ at $4{ }^{\circ} \mathrm{C}$. Anemones were digested in proteinase $\mathrm{K}$ for $35 \mathrm{~min}$ (45 min and longer resulted in sloughing off sensory cells from epidermis) and pre-hybridized overnight at $63^{\circ} \mathrm{C}$ with hybridization buffer including salmon sperm. The DIG-labeled RNA probes with a concentration of $1 \mathrm{ng} / \mathrm{ul}$ in hybridization buffer with salmon sperm were hybridized at $63^{\circ} \mathrm{C}$ up to 2 days (minimum of overnight).

Development took part with the enzymatic reaction of NBT-BCIP as substrate for the alkaline phosphatase conjugated anti-DIG antibody. Samples were developed until purple precipitate was visible as dependent on each probe, development stopped and samples were washed before mounting. Whole anemones were mounted in $80 \%$ glycerol solution on glass slides. Fixed and developed anemones were viewed under bright-field using a 
Leica DM5500 B microscope. Images were taken using a Leica DFC310 FX C-Mount 0.70x camera attachment and white balance restored in Adobe Photoshop.

\subsection{Results}

4.5.1 Identifiable anatomy in whole mount Exaiptasia pallida anemones: Anatomy, tissues, and cell types of the anemones were classified to identify the types of cells gluRs were expressed within or around. Anemones varied in size from 0.5 to $1 \mathrm{~mm}$ in length with oral diameters measuring from 0.2 to $0.4 \mathrm{~mm}$. Tissue and cell types were visible within E. pallida from 200x to 1000x magnification (Fig. 4.1). Orange-red colored carotenoids are present in tentacles and the body column of some anemones (Fig. 4.1FG). Carotenoids, associated with coral bleaching (loss of Symbiodiniaceae), are known for antioxidant responses as oxidative stress resistance within cnidarians were present in some of the anemones $(26,33,34)$. Symbiodiniaceae, algal-dinoflagellates that form an endosymbiosis with cnidarians (7), have diameters of approximately 10 um and clearly located within tentacles and some tissues within the body column (Fig. 4.1B and D) (3537). Acontinia, mesenterial filaments packed with mastigophores and isorhizas were visible in the column (Fig. 4.1D).

Tentacles have a variety of cell types, which were differentiated at 1000x (Fig. 4.1). Morphology was used to idententify sensory cells within tentacles of E. pallida (Fig. 4.1). Sensory cells that an apical cilium or a ciliary cone provide a point of contact to the external environment, similar to nematocytes, are closely associated with nematocytes 
and spirocytes that run along a nerve plexus (20). The general term for cnidarian nematocytes and spirocytes is cnidocyte $(38,39)$, which provides the namesake for the phylum Cnidaria. A cnidocyte contains a single cnida, the organelle known as a nematocyst or spirocyst (in general cnidocyst) $(26,27)$ that launches the microtubule attached to the stylet. Once nematocysts are discharged out of the cell, the long microtubules are visible outside of the cell. There are two main groups of nematocytes: mastigophores that are within long oblong shaped cells distinguished by a prominent central shaft (Fig. 4.1B) (26) and basitrich isorhizas that have a less noticeable central shaft $(25,27,40)$. In some images, tubules from mastigophores can be seen outside, as cnidae were ejected (Fig. 4.1C). The smaller defensive cells are spirocytes containing spirocysts, which lack a physical trigger hair or stereocilia. Spirocytes are distinguished by thin-walled spirocysts that have a visible accordion-pleated spiral tubule (Fig. 4.1B and D; (24). To identify each of these cell types within the microscopy images allows gluRs localization and association to the system of sensory and signaling cells.

\subsubsection{Localization of gluR genes in whole mount Exaiptasia pallida: Expression of} nine gluRs (E. pallida iGluR genes) (Table 4.1) was localized in whole mount E. pallida anemones via chromogenic in situ hybridization. Between concentrations of $1 \mathrm{ng} / \mathrm{ul}$ and 8 $\mathrm{ng} / \mathrm{ul}$, RNA probe concentration of $1 \mathrm{ng} / \mathrm{ul}$ provided the best resolution for in situ hybridization in the experimental pilot. Sense probe controls had rare false positive occurrence and presented little background noise across replicates (Fig. 4.2 and 4.3). For each gene, at least four biological replicates for antisense probes and three for sense probes were completed to demonstrate reproducibility (Table 4.3). 
Expression of gluR was confinedto the epidermal tissue layers (Fig. 4.6). Patterns of gluR expression differed between tentacles and column (Table 4.3, Fig. 4.2). There are several patterns that present more strongly than others. Localization occurred mostly within tentacles and adjacent to sensory cells. Expression of gluRs .1, .2, 4, 8, 10, 15, 21 and 22 displayed tentacles with more pigment relative to the body (Fig. 4.2). At increased magnification, gluR .1, .2, 8, 15, and 21 expression was concentrated at tips of tentacles (Fig. 4.3). Punctate patterns within tentacles occurred for gluR4 (Fig. 4.4), gluR10 (Fig. 4.3E), and gluR22 (Fig. 4.3I). The overall expression of gluR12 appeared less concentrated at 1000x magnification relative to the other gluR genes and was recurrent throughout the column of the polyp (Fig. 4.3F and 4.9). There was no gluR expression around the 10 um Symbiodiniacea located within the gastrodermal layer (Fig. 4.3 and 4.4) $(7,35)$. Localization of gluR expression was not detected within the internal gastrodermal tissues of the column or tentacles. The expression of E. pallida gluRs was not localized around carotenoids within the gastroderm (Fig. 4.1F and G), but gluR4 did express around the acontium where mastigophores are located (Fig. 4.7C). Considering the pattern of expression within the anemone column, some gluRs have evenly distributed probe localization across the entire body to the column of the polyps (Table 4.3, Fig. 4.6 and 4.10).

Polyp column staining was less prominent within chromogenic ISH of gluR mRNA expression. Expression within the column of the polyp (Fig. 4.2) occurs more prominently in gluRs 10,12 , and 22 where localized points of expression are scattered 
throughout tissue (Fig. 4.2, Table 4.3). Gene expression of gluR8 was quite sparse in the polyp column relative to the punctate pattern of gluR12 throughout tentacles in addition to the column (Fig. 4.8 and 4.9).

4.5.3 RNA localization of gluRs in relation to sensory cells: Expression of gluRs was confined to the epidermal cell layer of tentacles, which is the tissue layer that contains sensory cells i.e., nematocysts and spirocyts (Fig. $4.3 \& 4.6$ ). Along tentacles, gluR.1 was evenly dispersed and expression localized to sensory related cells, mastigophores (Fig. 4.4), while expression of gluR4 has sparsely punctate expression (Fig. 4.5). The homolog gluR4 was also expressed in tissues around the pedal disc of the anemone, the mastigophore-filled acontium, and potentially a dissociated neural cell (Fig. 4.7). Expression of gluR4 was also confined to the tips of mastigophores (Fig. 4.5). In contrast to mastigophores, spirocytes tend to be more abundant in tentacles than in the column (24), which may contribute to the pattern of gluR expression isolated to the tentacles. The high concentration in the tentacles for gluRs .1, .2, 15, 21, and 22 implicates gluR localization to spirocytes (Fig. 4.3). On the contrary, gluRs 4, 8, 10 and 12 present patterns evenly spaced, with punctate expression (Fig. 4.3). Relatively lower number of positive cells presenting gluR expression may account for less density of nematocytes versus spirocytes within the tentacles.

The punctate patterns of expression across the body column may also indicate gluR expression follow a path along the nerve net throughout the anemone (13). The gluRs that are expressed in this pattern, including gluR4, opens the possibility to gluR 
expression within the neuroepiderm. The neuroepiderm is where the nerve cells are located below the most outer cells of the epidermal tissue layer (41). Expression points of gluR12 are evenly spaced along the epiderm of the polyp column (Fig. 4.9), which is consistent with the nerve network of anemones. False positives or non-specific background staining appeared to occur more commonly, albeit still to a minor extent, in gluR sense probes with corresponding antisense-localization to the column (Fig. 4.2F) and in those that presented staining along the polyp column (Fig. 4.2H-I, Table 4.3). Any staining from sense probes was used to compare with antisense whole mounts staining to account for background or non-specific binding.

\subsection{Discussion}

The expression of E. pallida mRNA occurred within the epidermal tissue layers, localizing to the tentacles and to a more limited extent to the column (Fig. 4.1-2), which is consistent with the hypothesis that iGluR variants can functional in different roles $(16,42-46)$. Expression of gluRs on tentacles and sensory cells was hypothesized from localization of sensory-associated gene expression through cnidarian development $(13,31,47-50)$ and presence of iGluRs from genomic evidence in anthozoan species $(51,52)$. Signals occuring via synapses within sensory cells are found to perpetuate signals along the nerve plexus within cnidarians $(20,23,24)$. We inferred gluRs were likely functioning in or alongside specific sensory cells, potentially within nematocytes and cnidocytes $(25,27,29,41)$ from characterizational evidence (Ch. 2$)$ on the plausibility of glutamate and iGluRs in cnidocyte defense (27). Resulting expression of gluRs around sensory cells, including nematocytes and spirocytes, followed the hypothesized 
localization as deduced from findings of iGluR function and within developing anthozoans related to E. pallida.

Expression of gluRs throughout tentacles is consistent with spirocysts using chemical triggers rather than requiring physical contact to discharge $(24,25)$. We predict the rheostat determining where gluRs are expressed within anemone tentacles is related to chemosensation and physical triggering of spirocysts and nematocysts via these cnidocytes or neuro-synapse associated sensory cells. If gluRs require close proximal physical contact, then there is the increased likelihood of association with nematocytes that require cilia to act as as trigger. An alternative hypothesis is that these genes function in nerve net signaling. There is a pattern of expression that is similar to the path of the nerve net along the column of a polyp (Fig. 4.7A). As genes gluR4, gluR8, and gluR12 were chromogenically located throughout the entirety of the polyp (Fig. 4.8 and 4.9), the localization of expression throughout the polyp is consistent with a connection of gluRs to synaptic transmission within the nervous system.

It should also be considered that not all gluR genes within the genome have been localized (Ch. 2). Possibly these genes that have not been cloned from transcripts have wider localization within the nerve net. However, subfamily or class did not appear to be a determinant in localization of gluRs, which was considered to as a possibility for expression patterns from correlations between iGluR lineage and specific functions atune with cell type. The majority of gluRs (aside from gluR21 GluN_cnid) belong to the subfamily of Epsilon iGluRs (Ch.2). While chromogenic ISH provides a reproducible 
protocol and foundation for localizing gluR RNA expression, it is necessary to pursue three-dimensional imaging using fluorescent in situ hybridization to more closely identify associated cells and overlap between gluR expression. Genes that were not uniformly expressed throughout an anemone potentially have a ligand response specific to different cells and tissues. The same concept can be proposed for all gluR genes with differential expression patterns and putative functions. Functional studies to determine the ligands that activate these gluRs are now needed. This will help the field separate which of these iGluRs are involved in primary responses to an external stimuli such as cnidocyte stinging cells, perpetuating neural signals within nerve net, or additional roles in the cnidarian nervous system.

\subsection{Conclusion}

Exaiptasia pallida gluR genes were localized to express adjacent to or within cells responsible for detecting and responding to chemical-specific environment cues, including stinging cells located within tentacles, but also punctate throughout the column of the polyps. Localization of gluRs within tentacles suggests iGluRs associate to spirocytes, while those expressed at less density and within the body column are predicted to have association to mastigophore nematocytes. As the expression of gluR genes is closely tied to these sensory cells that contain or are nearby glutamate-triggered mechanisms, iGluRs have a putative function in the discharge of nematocysts and spirocysts for hunting and protective mechanisms, along with neural signaling for the entire polyp. Close association of gluRs occurring along sensory cells supports their chemosensory nature within cnidarians. To further confirm the associated responses of 
cnidarian iGluRs we propose to inject Xenopus oocytes with E. pallida gluR mRNA for functional experiments to determine ligand specificity.

\subsection{Acknowledgements}

Funding for this research was provided through NSF EDGE grant \#1645164 (to MRL, VMW, MD, AG, JP). The Internal Review Board exempted the cloning protocol (IBC17-017).

Specal thanks to Baily Steinworth and Leslie Babonis of the Martindale lab at Whitney Lab in UF for sharing their in situ protocols. Thanks to Jason Pernell for the open dialogue on his experience with Exaiptasia in situ hybridization. Sincere gratitude to Matt DeGennaro and his lab, for opening their doors and providing the space and support to complete this project and portion of EGD's dissertation. Thank you to Marcela Nouzova and Erasmo Perera for support with microscopy. 


\subsection{Tables and Figures}

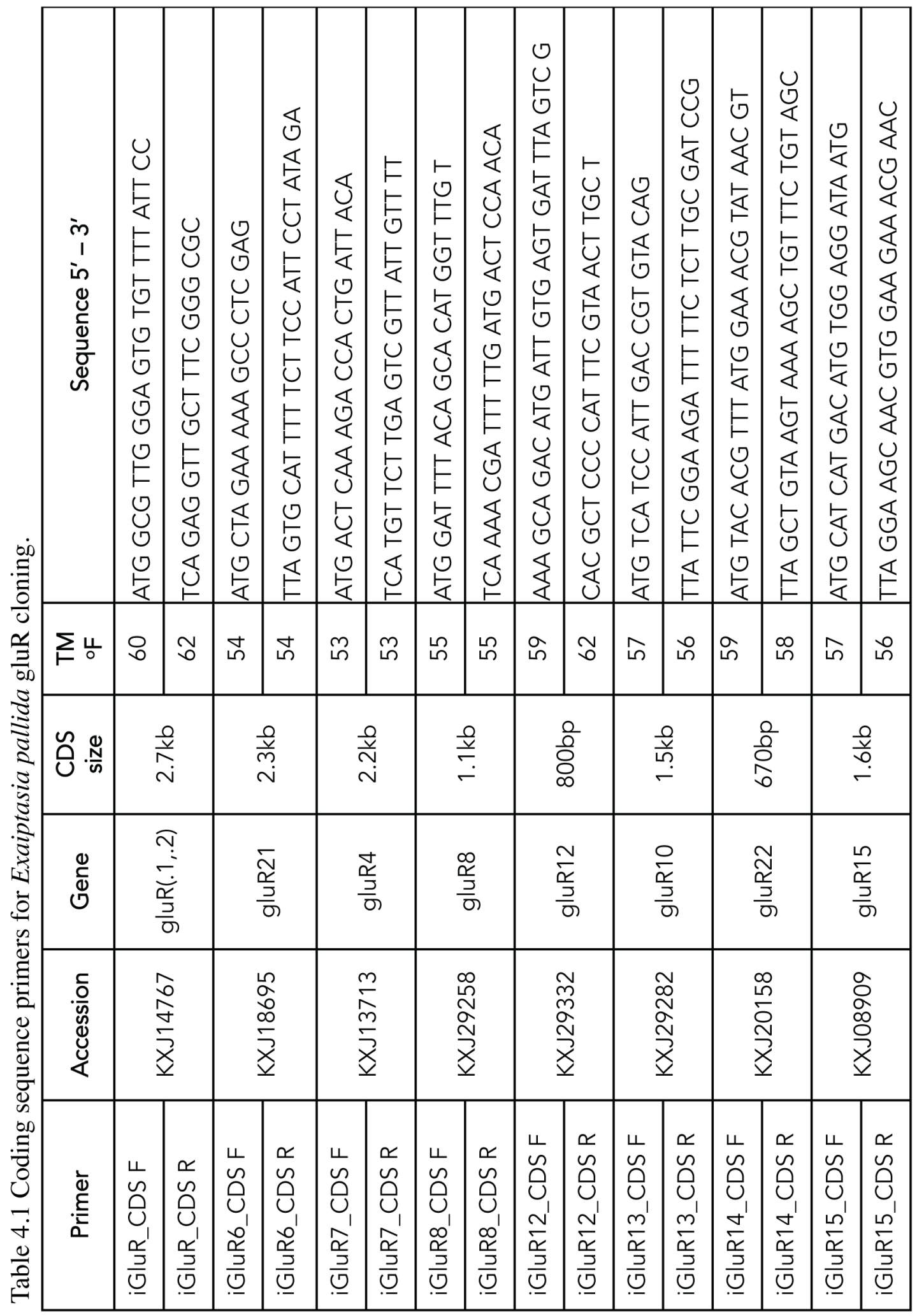


Table 4.2 Exaiptasia pallida gluR primers with pGEHME overhangs for directional assembly

\begin{tabular}{|c|c|c|}
\hline Primer & Gene & Sequence $5^{\prime}-3^{\prime}$ \\
\hline pGEMHE fwd & \multirow{2}{*}{ pGEMHE } & GAA TTC TCT AGA GCA AGC TTG ATC TGG TTA C \\
\hline pGEMHE rev & & GGA TCC CCG GGG AAT TGA TCT G \\
\hline iGluR fwd & \multirow{2}{*}{ KXJ14767 } & GAT CAA TTC CCC GGG GAT CCa tgg cgt tgg gag tgt gtt tta ttc \\
\hline iGluR rev & & CCA GAT CAA GCT TGC TCT AGA GAA TTC tta gag gtt tga ggg tcg gcc \\
\hline iGluR6 fwd & \multirow{2}{*}{ KXJ18695 } & GAT CAA TTC CCC GGG GAT Cca tgc tag aaa aag ccc tcg ag \\
\hline iGluR6 rev & & AAG CTT GCT CTA GAG AAT TCt tag tgc att ttt ctt cca ttc cta tag ata t \\
\hline iGluR7 fwd & \multirow{2}{*}{ KXJ13713 } & GAT CAA TTC CCC GGG GAT CCa tga ctc aaa gac cac tga tta cat tag \\
\hline iGluR7 rev & & CAA GCT TGC TCT AGA GAA TTC tca tgt tct tga gtc gtt att gtt ttt aga g \\
\hline iGluR8 fwd & \multirow{2}{*}{ KXJ29258 } & ATT CCC CGG GGA TCC atg gat ttt aca gca cat ggt ttg t \\
\hline iGluR8 rev & & TCT AGA GAA TTC tca aaa cga ttt ttg atg act cca aca tgc \\
\hline iGluR12 fwd & \multirow{2}{*}{ KXJ29332 } & TCC CCG GGG ATC Ccg gcc ccc aca cca ccg gtc att \\
\hline iGluR12 rev & & TTG CTC TAG AGA ATT Ctc acg ctc ccc att tcg taa ctt g \\
\hline iGluR13 fwd & \multirow{2}{*}{ KXJ29282 } & AAT TCC CCG GGG ATC Cat gtc atc cat tga ccg tgt aca g \\
\hline iGluR13 rev & & TAG AGA ATT Ctt att cgg aag att ttt ctc ttg cga tcc $g$ \\
\hline iGluR14 fwd & \multirow{2}{*}{ KXJ20158 } & CAG ATC AAT TCC CCG GGG ATC Cat gaa tct cgt tca atc tta cgt ctt tgt a \\
\hline iGluR14 rev & & AAG CTT GCT CTA GAG AAT TCc taa ttt gtg gct tgg gct cg \\
\hline iGluR15 fwd & \multirow{2}{*}{ KXJ08909 } & TCC CCG GGG ATC Cat gca tca tga cat gtg gag gat aat g \\
\hline iGluR15 fwd & & TGC TCT AGA GAA TTC tta gga agc aac gtg gaa gaa acg aac \\
\hline
\end{tabular}




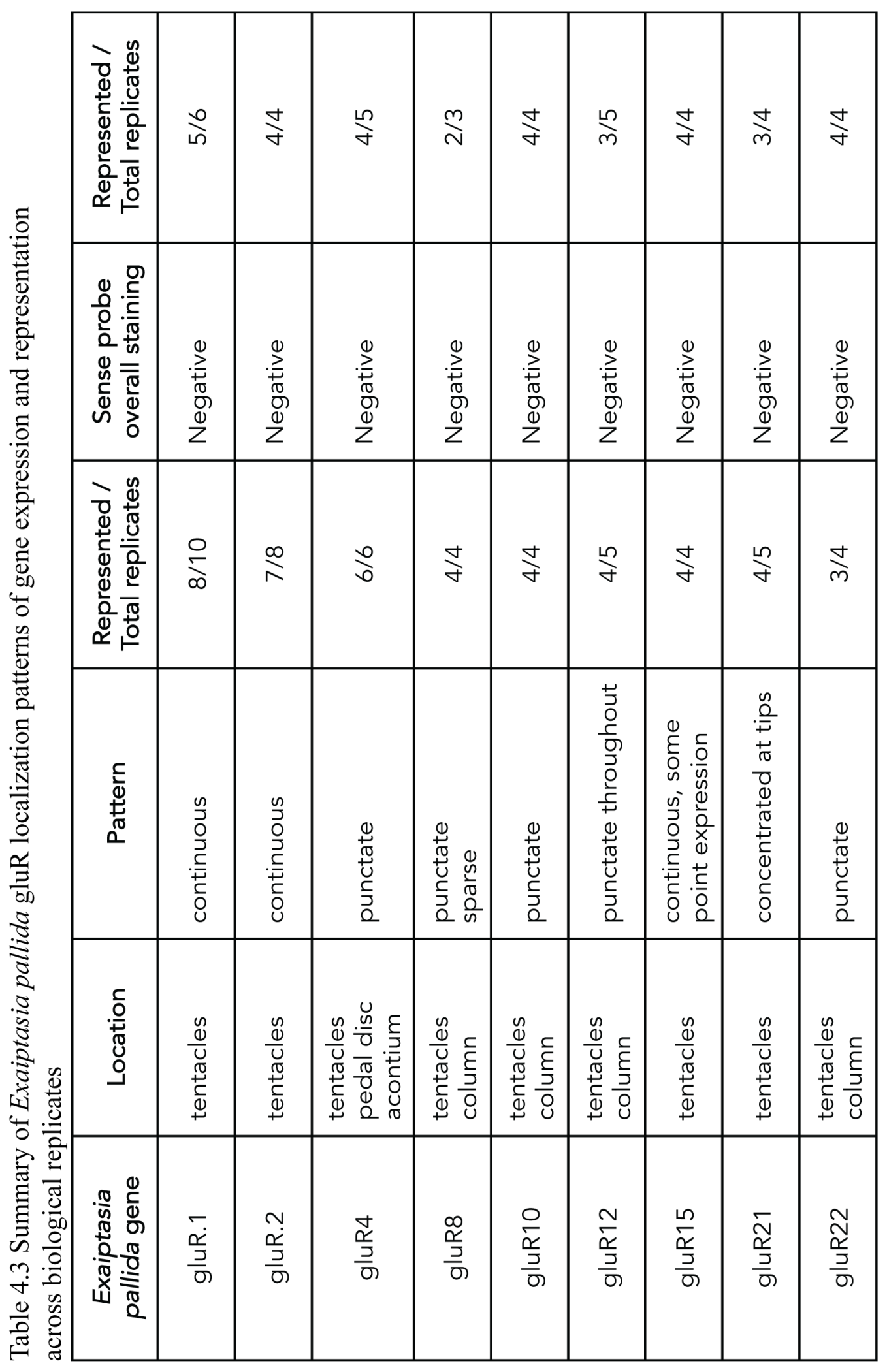




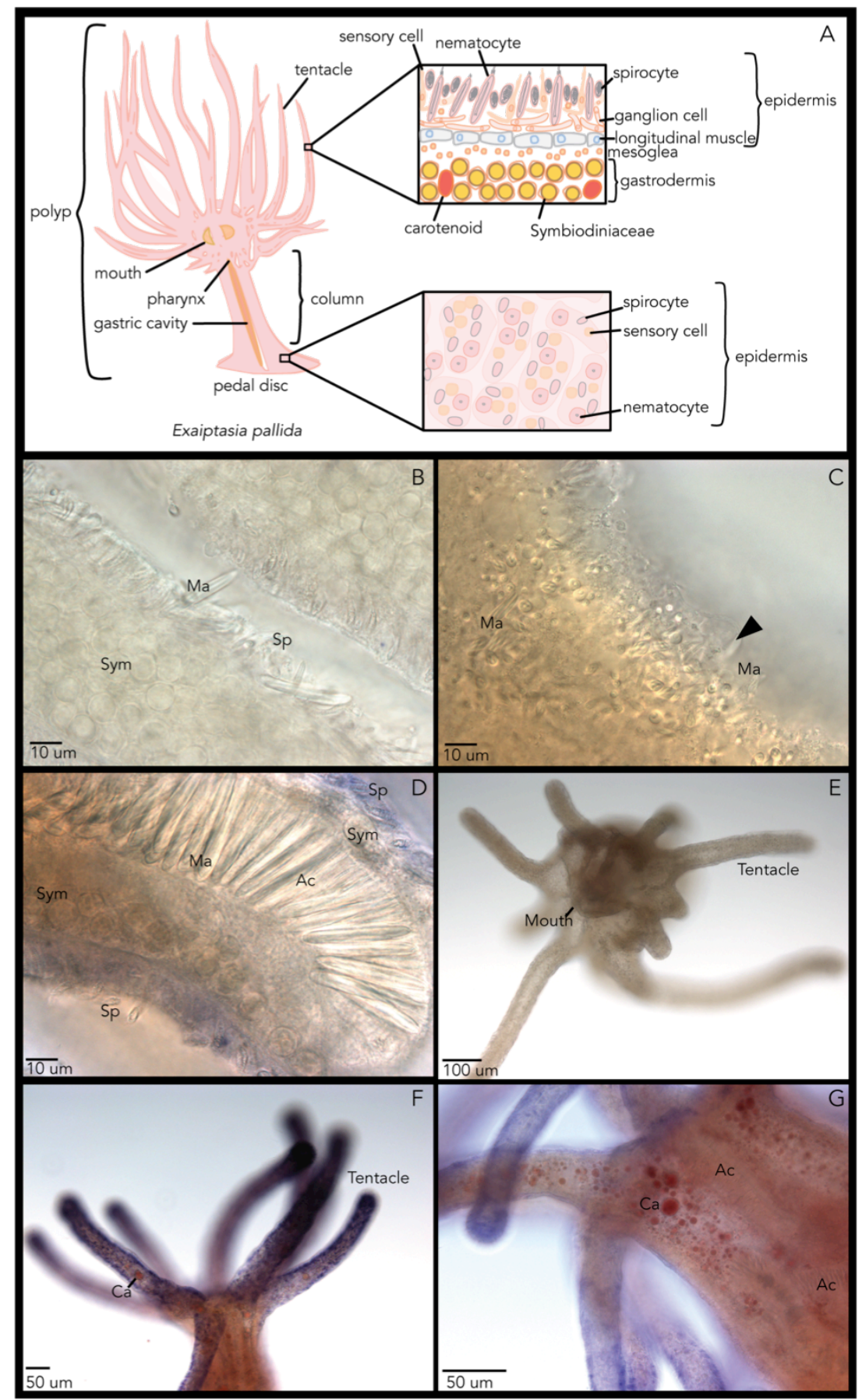

Figure 4.1 Cell differentation and anatomy of Exaiptasia pallida anemones.

A. Schematic of an anemone with anatomy labeled. Insets of tissue layers and cell differentiation. B. Tentacle filled with Symbiodiniaceae and lined with cnidae: nematocytes and spirocytes containing cnidocysts (nematocysts and spirocysts, respectively). C. Arrow pointing to launched tubule coil from a p-mastigophore on tentacle surface. D. Acontium packed with mastigophores. Visible spirocysts and mastigophores. E. Polyp from top view. F. gluR22 Antisense probe localization along the tentacles. Carotenoids present in anemone tentacles and body column. G. gluR.1 Antisense probe localization on tentacles and carotenoids within body and tentacles. ; $\mathrm{Ac}=$ acontium, $\mathrm{Ca}=$ carotenoid, $\mathrm{Sym}=$ Symbiodiniaceae, $\mathrm{Ma}=$ mastigophore, $\mathrm{Sp}=$ spirocyte, arrow=launched cnidae tubule 


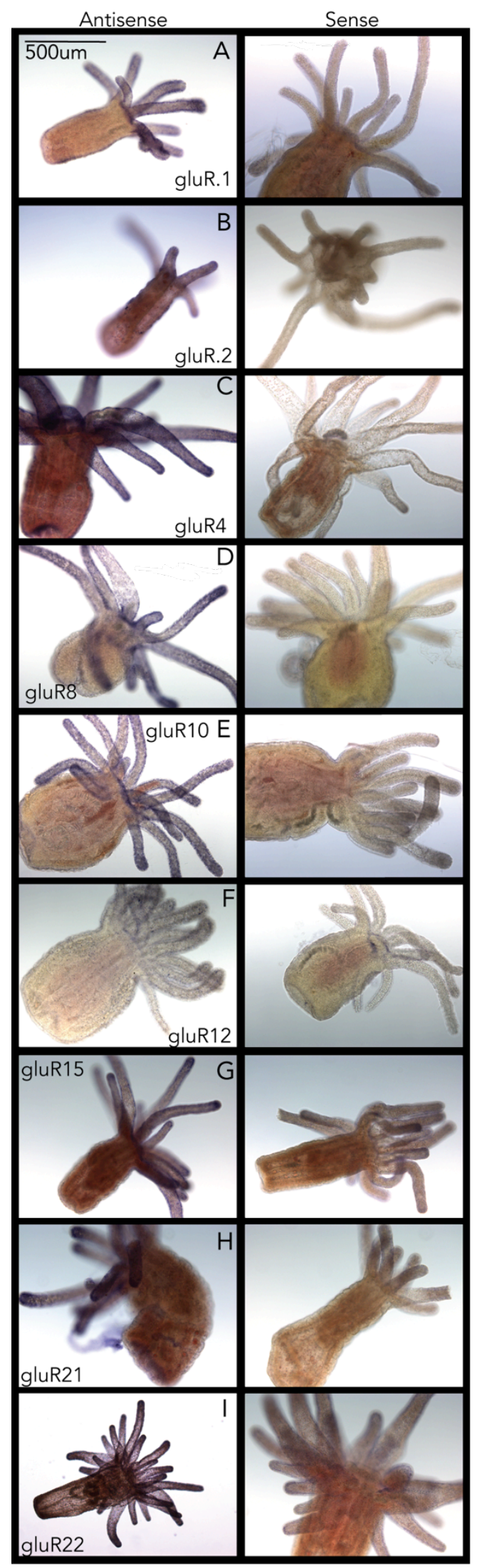

Figure 4.2 Whole mount in situ hybridization of Exaiptasia pallida anemones for gluR expression localization at $100 \mathrm{X}$ magnification under $70 \mathrm{X}$ camera magnification. Left antisense; right sense. Scale bar is $500 \mathrm{um}$. Representative images of A. gluR.1,

B. gluR.2, C. gluR4, D. gluR8, E. gluR10,

F. gluR12, G. gluR15, H. gluR21, I. gluR22 


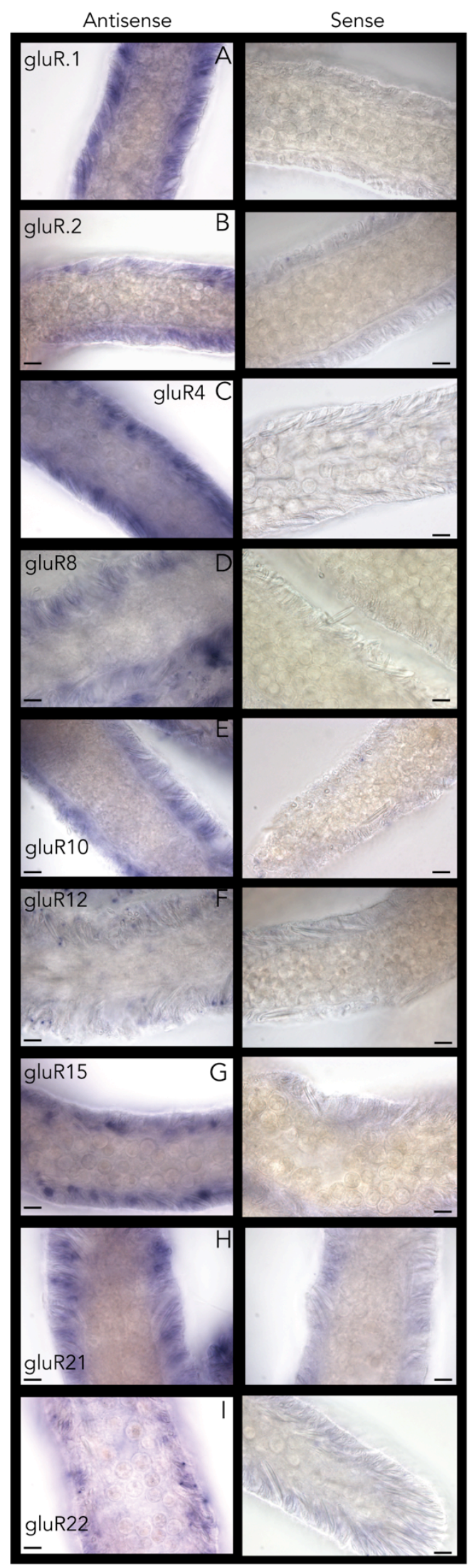

Figure 4.3 Tentacles of whole mount in situ hybridization of Exaiptasia pallida anemones for gluR expression localization at $1000 \mathrm{X}$ magnification under $70 \mathrm{X}$ camera magnification. Left antisense; right sense. The scale bar inset is $10 \mathrm{um}$, approximate size of Symbiodiniaceae. Representative images of A. gluR.1, B. gluR.2, C. gluR4, D. gluR8, E. gluR10, F. gluR12, G. gluR15, H. gluR21, I. gluR22 


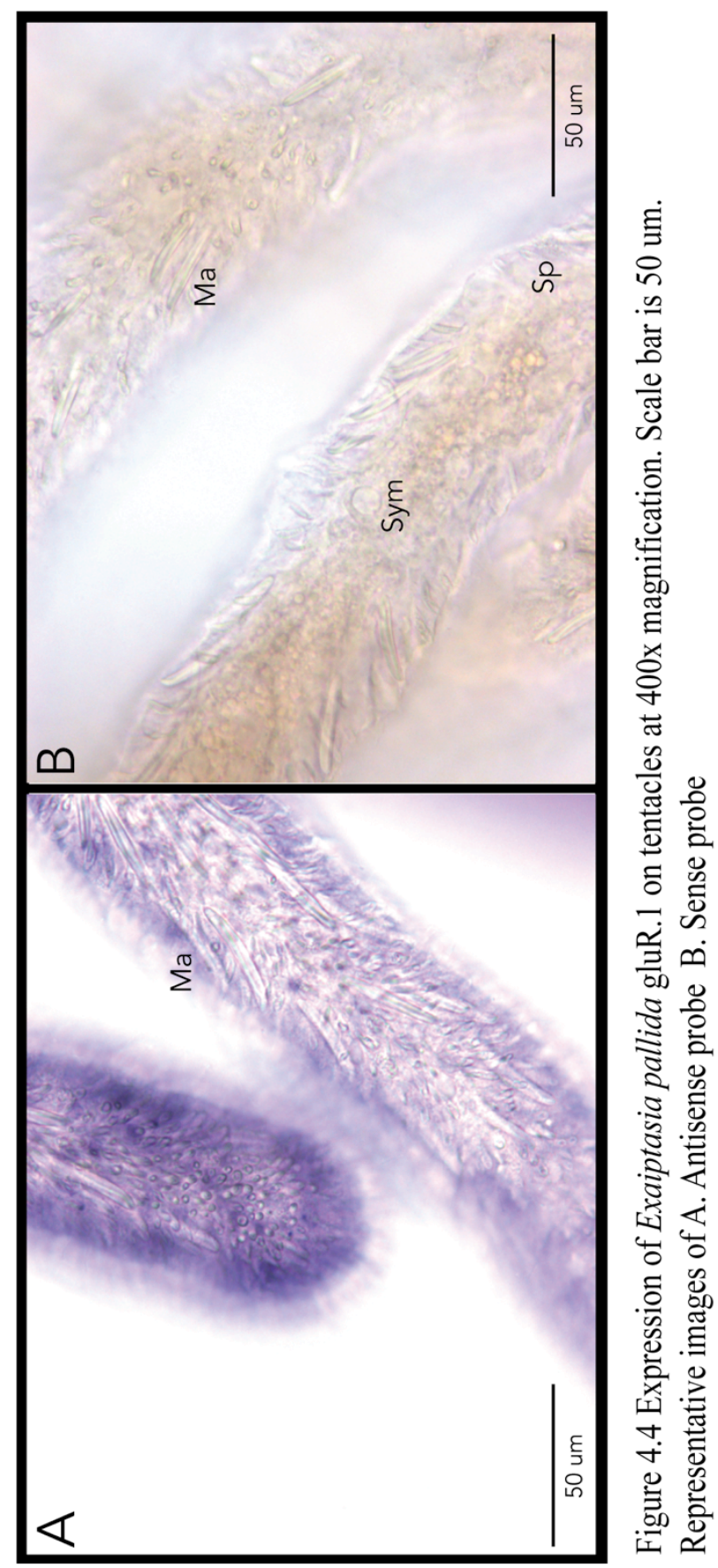




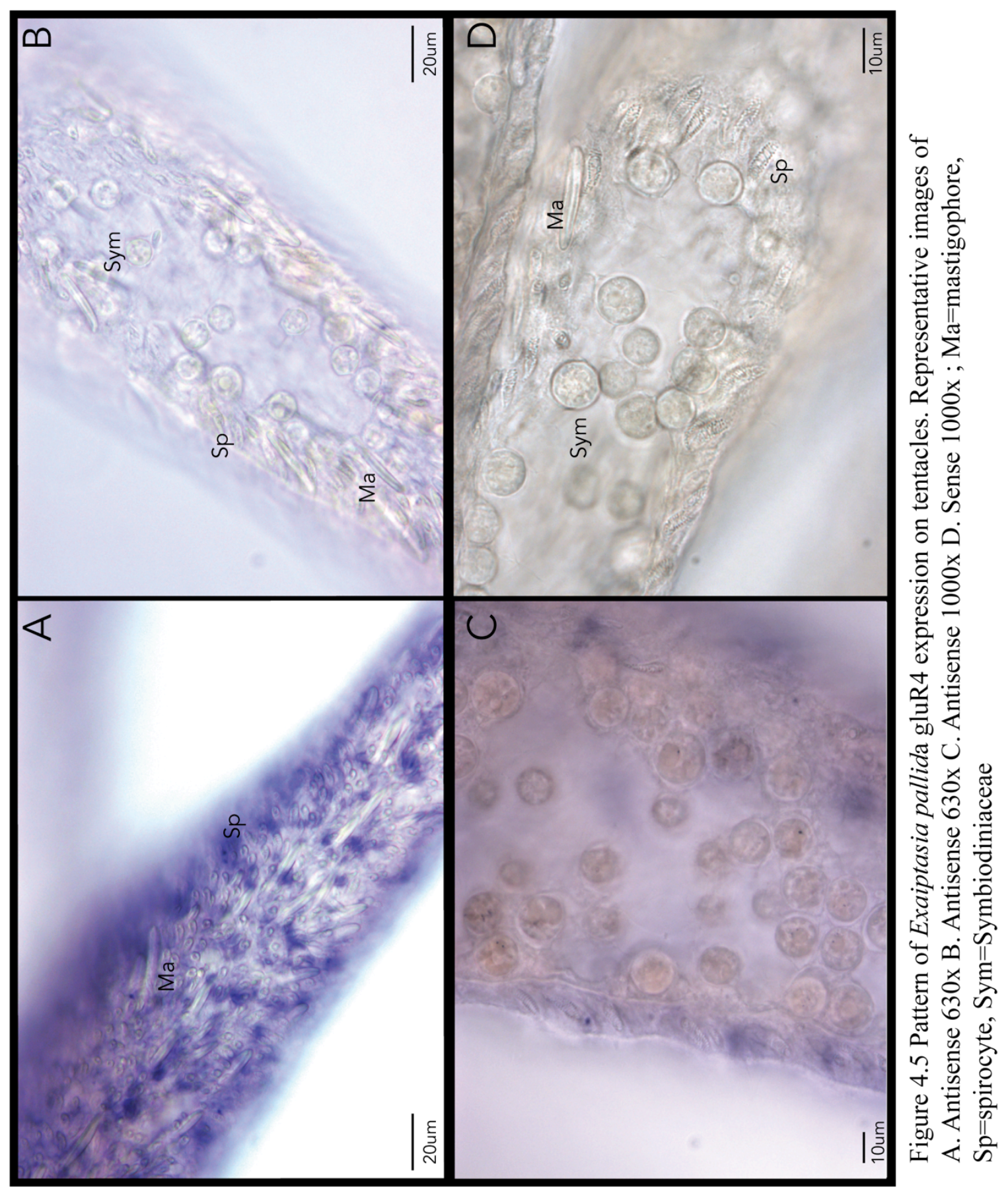




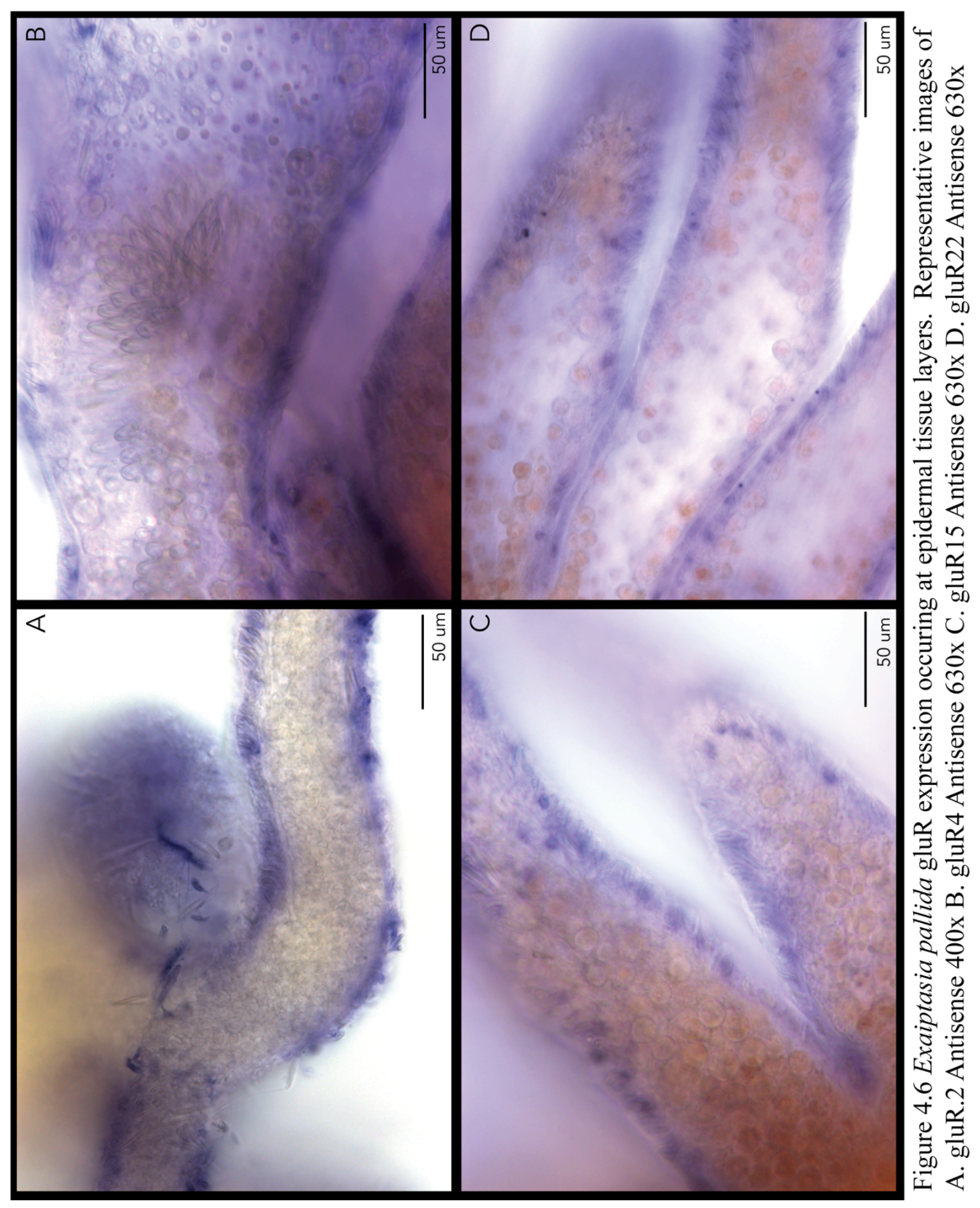




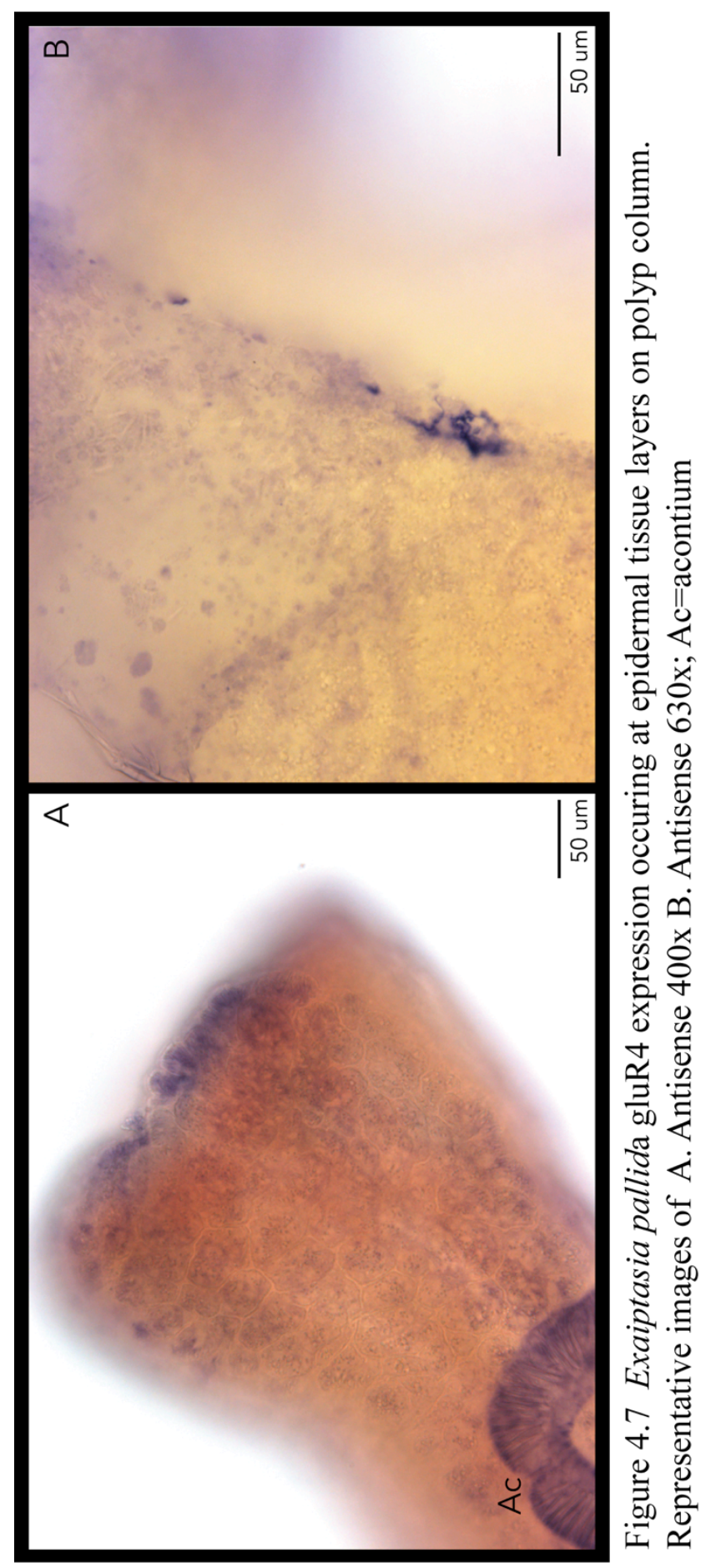




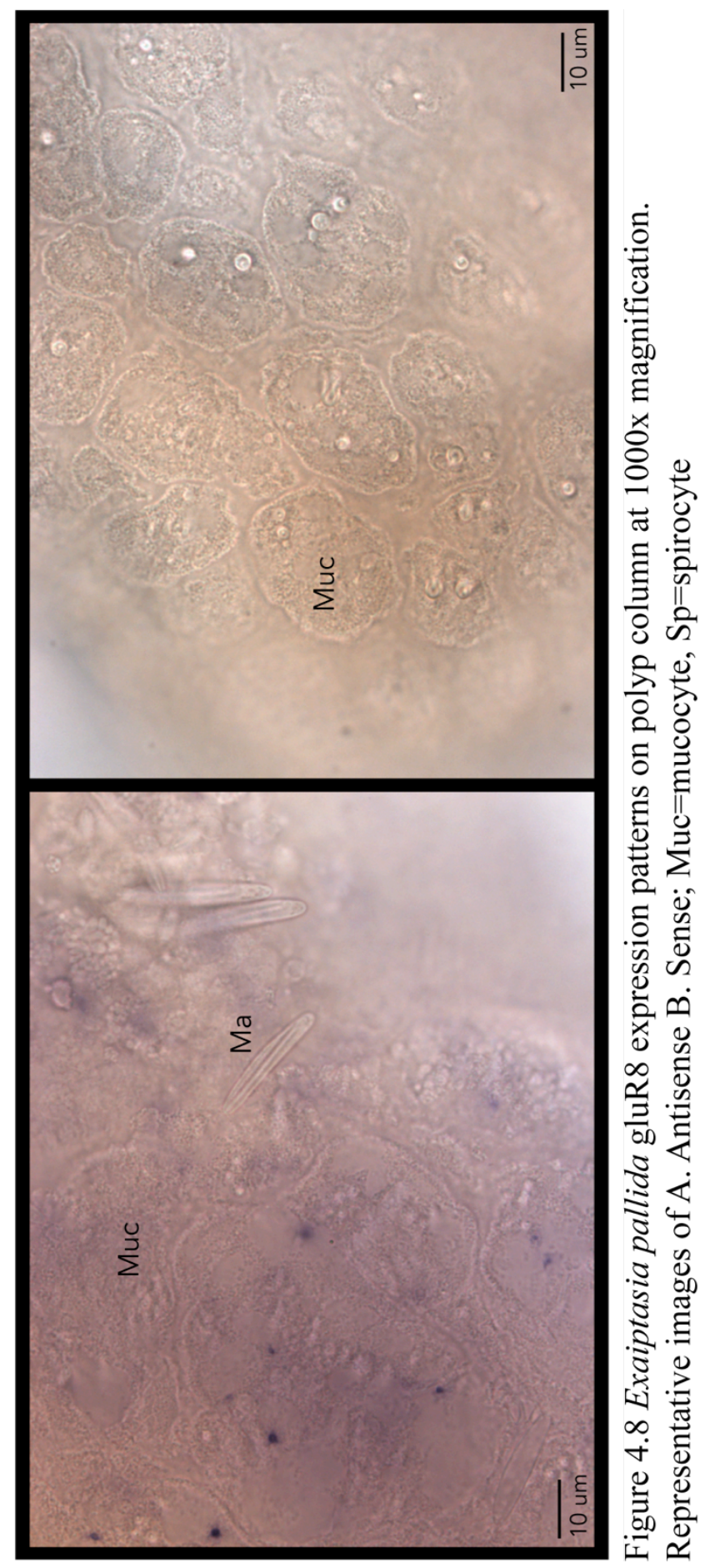




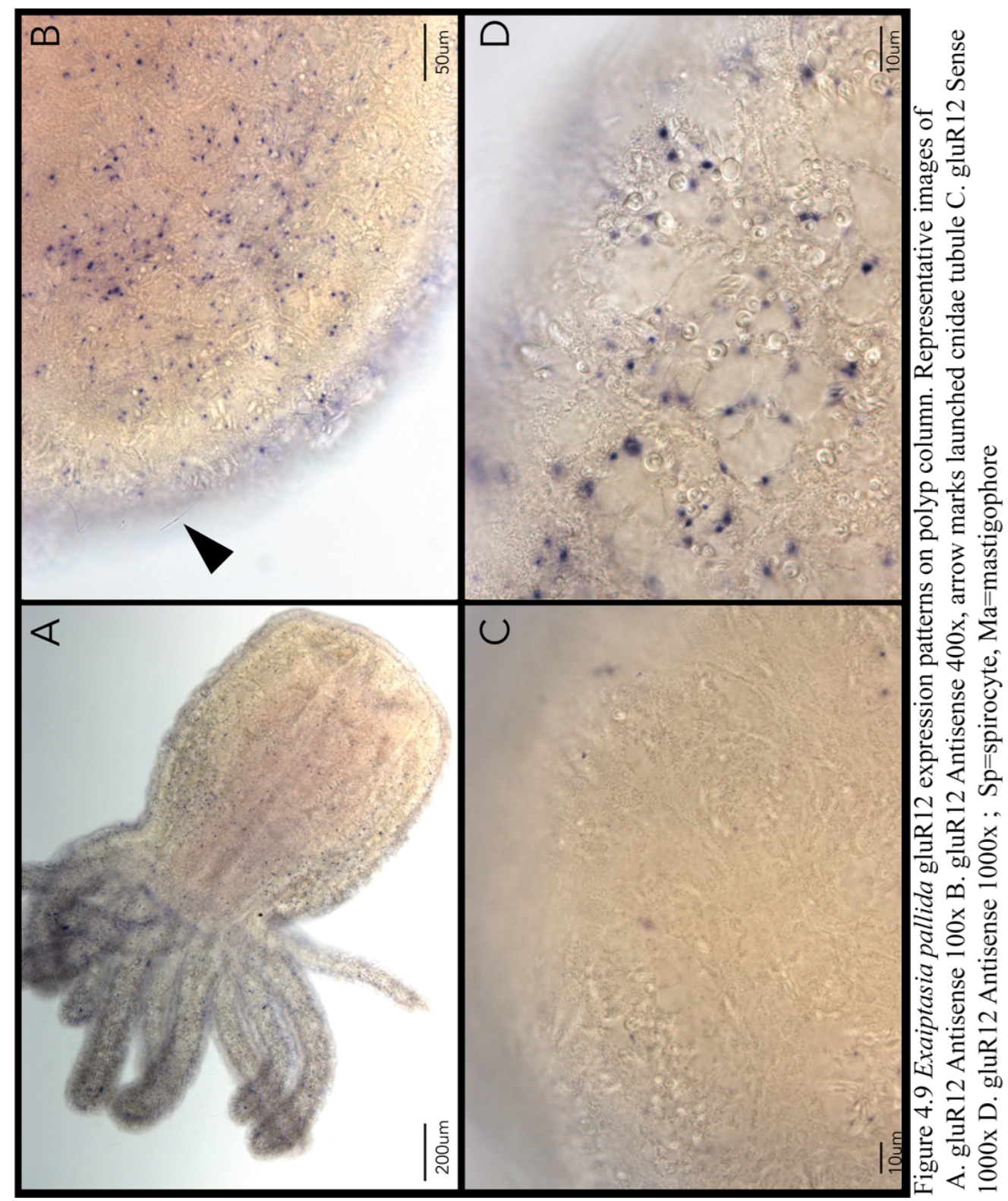




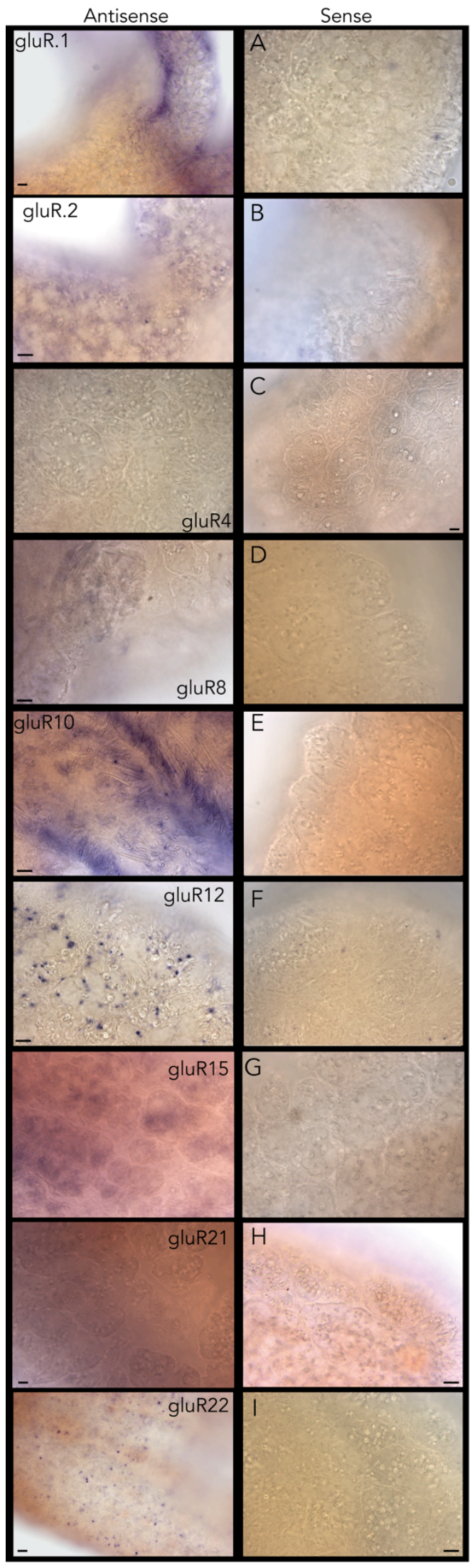

Figure 4.10 Expression of Exaiptasia pallida gluRs on polyp column of whole mount anemones. Viewed at $630 \mathrm{x}$ or $1000 \mathrm{X}$ magnification under $70 \mathrm{X}$ camera magnification. Left antisense; right sense. Scale bar set to 10um. Representative images of A. gluR.1, B. gluR.2, C. gluR4, D. gluR8, E. gluR10, F. gluR12, G. gluR15, H. gluR21, I. gluR22 


\subsection{References}

1. Zheng L, Zhang L, Lin H, McIntosh M, Malacrida A. Toll-like receptors in innate immunity. Int Immunol. 2005;17(1):1-14.

2. Miller DJ, Hemmrich G, Ball EE, Hayward DC, Khalturin K, Funayama N, et al. The innate immune repertoire in Cnidaria - ancestral complexity and stochastic gene loss. Genome Biol. 2007;8(4):R59.

3. Bosch TCG, Augustin R, Anton-Erxleben F, Fraune S, Hemmrich G, Zill H, et al. Uncovering the evolutionary history of innate immunity: The simple metazoan Hydra uses epithelial cells for host defence. Dev Comp Immunol. 2009;33:55969.

4. Hemmrich G, Miller DJ, Bosch TCG. The evolution of immunity : a low-life perspective. Trends Immunol. 2007;28(10).

5. Dunn S. Immunorecognition and immunoreceptors in the Cnidaria. ISJ. 2009;6:714.

6. Detournay O, Schnitzler CE, Poole A, Weis VM. Regulation of cnidariandinoflagellate mutualisms: Evidence that activation of a host TGF $\beta$ innate immune pathway promotes tolerance of the symbiont. Dev Comp Immunol. 2012;38:52537.

7. Palmer C V., Traylor-Knowles NG, Willis BL, Bythell JC. Corals use similar immune cells and wound-healing processes as those of higher organisms. PLoS One. $2011 ; 6(8): \mathrm{e} 23992$.

8. Müller WA, Leitz T. Metamorphosis in the Cnidaria. Can J Zool. 2002;80:175571.

9. Oren M, Paz G, Douek J, Rosner A, Amar KO, Rinkevich B. Marine invertebrates cross phyla comparisons reveal highly conserved immune machinery. Immunobiology. 2013;218:484-95.

10. Barr JJ, Auro R, Furlan M, Whiteson KL, Erb ML, Pogliano J, et al. Bacteriophage adhering to mucus provide a non-host-derived immunity Jeremy. PNAS. 2013;

11. Rentzsch F, Layden M, Manuel M. The cellular and molecular basis of cnidarian neurogenesis. Wiley Interdiscip Rev Dev Biol. 2017;6(1):1-19.

12. Watanabe H, Fujisawa T, Holstein TW. Cnidarians and the evolutionary origin of the nervous system. Dev Growth Differ. 2009;51:167-83. 
13. Furness JB, Stebbing MJ. The first brain: Species comparisons and evolutionary implications for the enteric and central nervous systems. Neurogastroenterol Motil. 2018 Feb 1;30(2):1-6.

14. Benton R, Vannice KS, Gomez-Diaz C, Vosshall LB. Variant Ionotropic Glutamate Receptors as Chemosensory Receptors in Drosophila. Cell. 2009;136(149-162).

15. Croset V, Rytz R, Cummins SF, Budd A, Brawand D, Kaessmann H, et al. Ancient protostome origin of chemosensory ionotropic glutamate receptors and the evolution of insect taste and olfaction. PLoS Genet. 2010;6(8):e1001064.

16. Abuin L, Bargeton B, Ulbrich MH, Isacoff EY, Kellenberger S, Benton R. Functional Architecture of Olfactory Ionotropic Glutamate Receptors. Neuron. 2011;69:44-60.

17. Liebeskind BJ, Hofmann HA, Hillis DM, Zakon HH. Evolution of Animal Neural Systems. Annu Rev Ecol Evol Syst. 2017;48:377-98.

18. Moroz LL. Convergent evolution of neural systems in ctenophores. J Exp Biol. 2015;218:598-611.

19. Westfall JA, Elliott CF. Ultrastructure of the Tentacle Nerve Plexus and Putative Neural Pathways in Sea Anemones. Biology (Basel). 2002;121(3):202-11.

20. Westfall JA, McCallum JD, Carlin RW. Neuroglandular synapses in the pharynx of the sea anemone Aiptasia pallida (Cnidaria, Anthozoa). J Morphol. $2001 \mathrm{Feb}$ $1 ; 247(2): 134-41$.

21. Saripalli LD, Westfall JA. Classification of Nerve Cells Dissociated From Tentacles of the Sea Anemone Calliactis parasitica. Ref Biol Bull. 1996;190:11124.

22. Westfall JA. Ultrastructure of systems synapses in the first-evolved nervous systems. J Neurocytol. 1996;25:735-46.

23. Westfall JA, Landers DD, McCallum JD. Ultrastructure of neuro-spirocyte synapses in the sea anemone Aiptasia pallida (Cnidaria, Anthozoa, Zoantharia). J Morphol. 1999 Aug 1;241(2):165-73.

24. Westfall JA. Neural pathways and innervation of cnidocytes in tentacles of sea anemones. Hydrobiologia. 2004;530/531:117-21.

25. Strömberg SM, Östman C. The cnidome and internal morphology of Lophelia pertusa (Linnaeus, 1758) (Cnidaria, Anthozoa). Acta Zool. 2017;98(2):191-213. 
26. Zbek S, Balasubramanian PG, Holstein TW. Cnidocyst structure and the biomechanics of discharge. Toxicon. 2009;54:1038-45.

27. Greenwood PG, Balboni IM, Lohmann C. A sea anemone's environment affects discharge of its isolated nematocysts. Comp Biochem Physiol Part A. 2003; 134:275-81.

28. Pierobon P. Coordinated modulation of cellular signaling through ligand-gated ion channels in Hydra vulgaris (Cnidaria, Hydrozoa). Int J Dev Biol. 2012;56(68):551-65.

29. Aranda PS, Lajoie DM, Jorcyk CL. Bleach Gel: A Simple Agarose Gel for Analyzing RNA Quality. Electrophoresis. 2012;33(2):366-9.

30. Wolenski FS, Layden MJ, Martindale MQ, Gilmore TD, Finnerty JR. Characterizing the spatiotemporal expression of RNAs and proteins in the starlet sea anemone, Nematostella vectensis. Nat Protoc. 2013;8(5):900-15.

31. Salinas-Saavedra M, Stephenson TQ, Dunn CW, Martindale MQ. Par system components are asymmetrically localized in ectodermal epithelia , but not during early development in the sea anemone Nematostella vectensis. Evodevo. 2015;120 .

32. Venn AA, Wilson MA, Trapido-Rosenthal HG, Keely BJ, Douglas AE. The impact of coral bleaching on the pigment profile of the symbiotic alga, Symbiodinium. Plant, Cell Environ. 2006;29:2133-42.

33. Galasso C, Corinaldesi C, Sansone C. Carotenoids from Marine Organisms: Biological Functions and Industrial Applications. Antioxidants. 2017;6(4):96.

34. Sunagar K, Columbus-Shenkar YY, Fridrich A, Gutkovich N, Aharoni R, Moran Y. Cell type-specific expression profiling unravels the development and evolution of stinging cells in sea anemone. BMC Biol. 2018;16(1):1-16.

35. Babonis LS, Martindale MQ. Old cell, new trick? Cnidocytes as a model for the evolution of novelty. Integr Comp Biol. 2014;54(4):714-22.

36. Westfall JA, Landers DD, McCallum JD. Different nematocytes have different synapses in the sea anemone Aiptasia pallida (Cnidaria, Anthozoa). J Morphol. 1998 Oct 1;238(1):53-62.

37. Wilkerson FP, Kobayashi D, Muscatine L. Mitotic index and size of symbiotic algae in Caribbean Reef corals. Coral Reefs. 1988;7(1):29-36. 
38. LaJeunesse TC, Parkinson JE, Gabrielson PW, Jeong HJ, Reimer JD, Voolstra CR, et al. Systematic Revision of Symbiodiniaceae Highlights the Antiquity and Diversity of Coral Endosymbionts. Curr Biol. 2018;28(16):2570-2580.e6.

39. Nakanishi N, Renfer E, Technau U, Rentzsch F. Nervous systems of the sea anemone Nematostella vectensis are generated by ectoderm and endoderm and shaped by distinct mechanisms. Development. 2012;139(2):347-57.

40. Collingridge GL, Lodge D, Mayer M, Turrigiano G, Frenguelli BG. Ionotropic glutamate receptors: Still exciting after all these years. Neuropharmacology. 2017;112:1-3.

41. Greer JB, Khuri S, Fieber LA. Phylogenetic analysis of ionotropic L- glutamate receptor genes in the Bilateria, with special notes on Aplysia californica. BMC Evol Biol. 2017;17(11).

42. Hodges G, Gutsell S, Taylor N, Brockmeier E, Butler E, Rendal C, et al. Invertebrate Model Species in AOP Development. In: Garcia-Reyero N, Murphy C, editors. A Systems Biology Approach to Advancing Adverse Outcome Pathways for Risk Assessment. Liverpool, UK: Springer International Publishing AG; 2018. p. 75-106.

43. Davenport R. Glutamate Receptors in Plants. Ann Bot. 2002;90:549-57.

44. Forde BG, Roberts MR. Glutamate receptor-like channels in plants: a role as amino acid sensors in plant defence? F1000Prime Rep. 2014;6(37):1-7.

45. Sinigaglia C, Busengdal H, Lerner A, Oliveri P, Rentzsch F. Evolution of Developmental Control Mechanisms Molecular characterization of the apical organ of the anthozoan Nematostella vectensis. Dev Biol. 2015;398:120-33.

46. Rentzsch F, Fritzenwanker JH, Scholz CB, Technau U. FGF signalling controls formation of the apical sensory organ in the cnidarian Nematostella vectensis. Development. 2008;135:1761-9.

47. Watanabe H. Back Through Time: How Cnidarians and Basal Metazoans Shed Light on Ancient Nervous Systems. In: Shigeno S, Murakami Y, Nomura T, editors. Diversity and Commonality in Animals: Brain Evolution by Design From Neural Origin to Cognitive Architecture. Japan: Springer, Diversity and Commonality in Animals; 2017. p. 45-75.

48. Saina M, Busengdal H, Sinigaglia C, Petrone L, Oliveri P, Rentzsch F, et al. A cnidarian homologue of an insect gustatory receptor functions in developmental body patterning. Nat Commun. 2015;6:6243. 
49. Ramos-Vicente D, Ji J, Gratacòs-Batlle E, Gou G, Reig-Viader R, Luís J, et al. Metazoan evolution of glutamate receptors reveals unreported phylogenetic groups and divergent lineage-specific events. Elife. 2018;7:e35774.

50. Krishnan A, Schiöth HB. The role of G protein-coupled receptors in the early evolution of neurotransmission and the nervous system. J Exp Biol. 2015;218:56271. 


\section{CHAPTER V. CONCLUSIONS AND SYNTHESIS}

\subsection{Synthesis of findings}

This project began as an investigation into the involvement of chemosensory proteins in an innate immunity-focused perspective and grew to encompass the study of the evolution and explore putative functions of chemosensory ionotropic glutamate receptors (iGluRs) within Cnidaria, explicitly using the model cnidarian, sea anemone Exaiptasia pallida (Agassiz in Verrill, 1864). The results of the findings presented through Data Chapters II-IV, have led to the development of several hypotheses on the evolutionary roles of neural networks and chemical signaling.

Chemosensation, or chemical sensing, is a fundamental signaling pathway correlating innate immunity and neural signaling within the anemone, E. pallida through chemosensory iGluRs. Polyphyletic E. pallida iGluRs are predicted to act as sensory receptors and respond to immune and environmental stimuli. This dissertation explored the diversity of iGluRs within cnidarians, measures expression of E. pallida iGluRs in response to two environmental stimuli, and chromogenic localization of expression in cells and tissues within whole E. pallida anemones. Several methods were used to characterize the iGluR protein family within Cnidaria. Phylogenetic analyses were used in the approach to determine the extent of cnidarian diversity across metazoans. Further bioinformatic analysis in homolog conservation was used as a starting point for predicting putative functions from protein functional domains. The available genome and transcriptome of E. pallida was important for both bioinformatics analysis and within the 
approach for gene expression. Bacterial challenges and diurnal cycling have implications of the environment in eliciting changes in expression of gluRs in E. pallida.

Chromogenic localization of genes is consistent with the hypothesis that gluR expression correlates to sensory cells and the nervous system in the putative function of iGluRs within Cnidaria.

\subsection{Chapter summaries}

The range of iGluR diversity in available genomes along with complementary data on putative cnidarian functions has given rise to hypotheses on the cnidarian role of iGluR function. Overall characterizations are summarized in Table 5.1. There were 22 unique genes coding for E. pallida GluR (one set of isoforms, named gluR.1 and gluR.2)

dispersed over four phylogenetic clades across subfamilies and classes of iGluRs (Ch. 2) with expansions in Cnidaria. Four of these genes from clades $\mathrm{C} 2$ and $\mathrm{C} 4$ had significant changes in expression, as a response to bacteria challenge, while two within $\mathrm{C} 4$ did not respond at all (Ch. 2). In addition to inference of GluN and GluE expression responses to bacteria, there was the underlying implication of time as a factor to investigate.

Two iGluRs differentiated in expression cycle when quantified via a diel zeitgeber, a $12 \mathrm{hr}$ light: $12 \mathrm{hr}$ dark cycle versus a $12 \mathrm{hr}$ dark: $12 \mathrm{hr}$ dark cycle sans light stimulus (Ch. 3). Exaiptasia pallida gluR.2 (isoform of GluR), a respondent to bacterial challenge, was calculated to have a 16-hr period, compared to an 8-hr period in LD cycle. Exaiptasia pallida gluR8, which had not responded to bacterial challenge, was calculated to have a 24-hr period within DD, referred to as a diel or circadian, compared to an 8-hr 
period in LD. While, we cannot claim gluR8 expression has a circadian rhythm, gluR expression changes are consistent with changes in transcriptomic data of iGluRs from the symbiotic relationship with algal-dinoflagellates and in Exaiptasia diaphana (Rapp,

1829) (Ch. 3) (1). The expression changes in sea anemones from symbionts and diurnal fluctuations imply the metabolic outputs of Symbiodiniacea via primary productivity affect transcription of iGluRs (Fig. 5.1).

Expression of E. pallida gluR genes was localized mostly to E. pallida tentacles, where nematocysts and sensory cells reside, while some gluRs were expressed throughout the column of anemone polyps (Ch. 4). The expression of gluRs to only tentacles implies the sensory proteins align to spirocysts or nematocysts, which is consistent with the connections of glutamate to cnidocyte firing (2,3). Exaiptasia pallida gluRs that did not have an expression response to bacteria are potentially associated to internal nervous system signaling. It is possible that different $E$. pallida gluRs are prescribed for different functional roles from synapses to environmental receptors, dependent on their association with sensory cells.

\subsection{Exploring ligand specificity}

Phylogenetic and structural analyses suggest identified E. pallida iGluRs maintain functional domains of chemosensory proteins (Ch. 2). Coupling bioinformatics with transcriptional data implies iGluRs have a putative function in the context of bacterial defense (Ch. 2). The GluR sequences from E. pallida span several clades, designating $E$. pallida iGluRs as polyphyletic. From our knowledge of classical iGluRs, we know this 
family of receptor proteins diverges into different clades based on their specificity of synthesized ligands. Drawing this parallel, we can hypothesize that each of these clades of E. pallida iGluRs (C1-C4) has a specific ligand (or several ligands), which requires further investigation to confirm. While the ligand-binding domain may detect glutamate or glycine or a bacterial-specific ligand site, we do not know at this stage as to which specific chemical each GluR subunit responds. Ligand specificity for different iGluR subunits have been documented as GluN1, GluN3, and GluD binding glycine and Dserine, while GluA and GluK bind glutamate (4-6). I hypothesize GluR subunits classified in $\mathrm{C} 1$ putatively respond to NMDA and glycine, GluRs within $\mathrm{C} 2$ to glycine and D-serine, and GluRs within C3 and C4 to glycine and/or glutamate. Exaiptaisa pallida iGluRs within $\mathrm{C} 2$ and $\mathrm{C} 4$ cnidarian subclades are conjectured to demonstrate cnidarian-specific ligand binding associations within GluN and GluE subfamilies, which is consistent with species-specific ligand specificity within respective expansions. Ligand specificity within species expansions has been demonstrated in ctenophore binding specificity toward glycine (7).

A standard method to assess ligand specificity is through measuring ion channel activity via expressing ion channel proteins within Xenopus oocytes $(8,9)$. Xenopus oocytes are standard to use as model cells for in vivo experimentation; the oocytes can be de-folliculated from the ovarian sacs and logistically important, are relatively large in size (8). A large cell size is ideal for studying ion channels as a two-electrode clamp can fitted on the cell to measure extracellular currents flowing through the membrane with a vibrating probe (9). This approach would use full-length E. pallida cDNA sequences of 
iGluRs within a pGEHME vector (provided from samples preserved during Ch. 4) to be transcribed into mRNA in vitro or synthesized into cRNA, and injected into Xenopus oocytes for expression, ultimately isolating the receptors individually or in co-receptor pairs to measure ligand response. Through this experimental system the selected iGluR proteins can be expressed alone or in specified combinations. To measure activity of the iGluRs, oocytes expressing receptors of interest would be exposed to candidate ligands related to immunity and chemosensation for ligand-specific measurements of electrophysiological response. Specific ligands that will be targeted include various known agonists and antagonists i.e., AMPA, NMDA, kainate, L-glutamic acid, L-aspartic acid, glutamate, glycine, D-serine and DNQX $(5,10)$ and immunological agents of $V$. coralliilyticus, S. marcescens, lipopolysaccharide, and peptidoglycan. Data from ligand specificity would allow us to confirm accuracy of the naming conventions used for iGluRs in applicability to invertebrates and more importantly, determine functional responses and the role of iGluRs within chemosensory pathways.

\subsection{Putative functions of Exaiptasia pallida iGluRs}

Initial exploration into gluR putative functions occurred through developing an immune challenge experiment using two bacteria, Vibrio coralliilyticus and Serratia marcescens (Ch. 2). It should be noted here the GluR with two isoforms, gluR.1 and gluR.2, responded to $S$. marcescens. In response to $V$. coralliilyticus, gluR.2 displayed the strongest transcriptional changes compared to the other gluR genes. Also from $\mathrm{C} 4, E$. pallida gluR4 and gluR8 did not have a significant response to the bacterial challenges. However, bacterial treatment did significantly impact C2 gluR21 and C3 gluR23. Across 
$\mathrm{C} 2-\mathrm{C} 4$, there is a bacterial response, but this does not mean every E. pallida gluR gene will respond to this stimulus. Sampling over the course of several days demonstrated that the 24-hour time point is the critical to the affect of time.

I further explored the expression of the bacteria-responsive gluR.2 and nonresponsive gluR8. Within diurnal experimentation via light cycling E. pallida gluR.2 and gluR8 expressed ultradian 8-hr periods under normal light:dark conditions. The period changed for both expression patterns of gluR genes when compared to anemones in constant darkness; implying E. pallida iGluRs have different expression cycles without a diurnal signal. Symbiosis tightly connects to diurnal cycles in cnidarians with endosymbiotic Symbiodiniaceae (11-13). Recent expression data from the facultativesymbiotic anemone Exaiptasia diaphana suggested iGluRs have different cycles dependent on the presence of symbionts (1). Transcriptional regulation of iGluR gene expression was consistent with altering from a symbiosis-associated 24-hr period to aposymbiotic-associated 12-hr period. While the pattern did not hold fast for every iGluR sequence, expression cycles consistently differed between symbiotic states for the anemones. Endosymbiotic Symbiodiniaceae rely on light for metabolism via primary productivity, which changes from day to night. When I interrupted the light cycle during the diurnal experiment (Ch. 3), I uncovered a potential endogenous period cycle for the E. pallida iGluRs. The rhythm would only be endogenous if it is circadian. Regardless, removing light changed iGluR transcription, which was consistent with iGluR transcription changes to Symbiodiniacea in E. diaphana. 
Expression data is not complete for the entirety of all E. pallida iGluRs, because of financial and practical restrictions including the number of individual anemones required for each experiment and consequent amount of processing. In addition to the number of iGluRs, there is also the consideration of the 24 -hr sample period versus a 48 hr period. Measuring expression over 48 hours would provide stronger support for the cycle of the biological rhythm (14), however, the aim of this experiment was to determine if there was a periodicity or factor of time in gluR expression. The most interesting finding for me within Chapter II was change in expression between light cycles and how this was consistent with the altering expression cycles of iGluRs from Symbiodiniacea (1). In order to demonstrate biological rhythms in E. pallida, a transcriptomics study sampling anemones at least every four hours over a 48-hr period with the experimental set up testing the factors of light as a diel factor or alternatively the presence and absence of Symbiodiniacea. In a transcriptomic study, the data could be explored to uncover iGluR genes and their expression cycles as I did with the E. diaphana data (1).

\subsection{Chromogenic localization of gluRs within Exaiptasia pallida}

To establish a chromogenic in situ hybridization protocol, I cloned and sequenced nine $E$. pallida iGluRs from cDNA. The E. pallida gluRs were inserted into a pGEMHEcontaining vector, which was necessary for the initially planned functional experiments. Each of these cloned genes was used to create probes specific to each gene. In situ localization has not always been successful within E. pallida, but I was able to develop a protocol and process nine probes, both sense and anti-sense, for characteristically lowexpressed receptors. Exaiptasia pallida gluRs were expressed for the most part within the 
tentacles of the whole mount anemones. I hypothesize the location of the E. pallida gluRs overlaps closely to sensory cells and defensive nematocytes and spirocytes. Anemones have a denser, higher concentration of spirocytes relative to nematocysts within tentacles. The genes expressed at high concentrations, total coverage of tentacles, are predicted to be expressed within or adjacent to spirocytes, while those with greater spacing between expression points within or adjacent to nematocytes. This idea is purely conjecture based on the concentrations of spirocytes versus nematocytes in anemone tissues $(3,15)$. However, the E. pallida iGluRs are expressed within the epidermal layer and not within the gastroderm. I originally hypothesized E. pallida iGluRs would be located within the gastroderm because of phagocytic cells and the initial connection of iGluRs to cnidarian innate immunity (16).

At this juncture, I hypothesize E. pallida iGluRs are more likely linked to neural signaling within the dispersed nervous system of the anemones. Anemones have variety of neural cells dispersed throughout a nerve net, that the anemone uses as communication across the polyp. Instead of having a CNS to process signals, anemones act purely on an immediate 'gut' reaction, quick signaling and identification of the biological or environmental elicitor that set off those signals via the nerve net. Perhaps in these earlydiverged metazoans, the large diversity across clades is necessary for more specificity and nuanced specificity between ligands. It is possible the E. pallida iGluRs act as coreceptors and forming various combinations of subunits, therefore, even with only four clades and 22 genes, it is possible there are many more potential ligands or specificity to different signals. 
To continue localizing gene expression of iGluRs, whole mount fluorescent in situ hybridization (FISH) can assess localization of iGluR gene expression $(17,18)$. FISH can be used to determine where iGluR genes are transcribed through fluorescent markers that bind to iGluR-specific probes. Co-expression can be determined by overlapping iGluR FISH with multiple probes and specific markers for each probe, which would provide potential pairs to inject into Xenopus oocytes. Then the ability to use RFamide to outline the cnidarian nervous system $(3,15,19)$ would allow overlap between labels to identify how closely E. pallida iGluRs associate to the nerve net.

\subsection{Ecological and applicable relevance of cnidarian iGluRs}

The primary goal of an organism is to maintain homeostasis, to stay within those set bounds for cellular operations. Changes within environment provoke behavioral changes or physiological responses, dependent on adaptations to environmental fluctuations. As anemones are sessile creatures, their responses are exclusively physiologically based and changing their internal environments. The most drastic physiological change cnidarians undergo is that disruption of symbiosis leading to coral bleaching, the event when polyp tissues either lose or eject Symbiodiniacea leaving bone-white ghostly coral frames. While unknown if these receptors are connected to bleaching, expression patterns of gluRs opens the possibility of influence by symbiosis. Their regulation changes in response to light and symbiotic state (Fig. 5.1). Alternating metabolic outputs, including extreme changes in $\mathrm{pH}$ and ion concentrations, may be the biochemical catalyst that impacts regulatory systems of E. pallida gluR expression within symbiotic anemones. 
This dissertation has led me throughout various methods to characterize a large family of signaling receptors. From bioinformatics to predictive applications, I characterized iGluRs within the genome of E. pallida and addressed putative involvement of several receptors. However, this is only a start to the understanding of the importance of cnidarian iGluRs, which is possible through the established genome. Through characterizing the E. pallida gluR genes, I developed hypotheses for predicted functions. In order to completely characterize these receptors, functional work across the entire phylogeny of E. pallida iGluRs needs to be completed.

To illustrate the role of these receptors within an invertebrate is important in relation to our knowledge of how iGluR dysfunction can lead to neurological issues and degeneration within humans. Cnidarians do not exhibit the aging process in the apparent way humans do, so perhaps through this experimental model we can learn more about how to better sustain iGluRs within the environment of an aging brain. An anemone is constantly communicating with the outside world, there is barely a barrier to define where the organism begins and ends between the symbioses with Symbiodiniaceae to associated bacteria to the biogeochemical components of the ocean. We contemplate how receptors unremittingly exposed to a changing environment and internal cycling in a sessile organism continue to work efficiently. Through continued research, we can observe iGluR involvement in development and predict the role within a nerve net that utilizes conductor and environmental sensory cells. We recognize these receptors are essential for various signaling functions; it is possible iGluRs have a putative function to 
maintain regulation and stability for homeostasis. From my dissertation research, I have opened several doors and explained how to continue exploration of these proteins and to learn how iGluRs function in cnidarians and their essential roles that required gene expansion. 


\subsection{Tables and Figures}

Table 5.1 Overview of Exaiptasia pallida iGluR characterization and findings.

\begin{tabular}{|c|c|c|c|c|c|}
\hline gluR & Clade & Class/Subfamily & Immune Response & Biological Rhythm & Location \\
\hline .1 & C4 & GluE_cnid & bacteria: Serratia & & tentacles \\
\hline .2 & C4 & GluE_cnid & bacteria: Serratia & LD 8-hr ; DD 16-hr & tentacles \\
\hline 3 & $\mathrm{C} 4$ & GluE_cnid & & & \\
\hline 4 & C4 & GluE_cnid & none & & tentacles, pedal disc, acontium \\
\hline 5 & C4 & GluE_cnid & & & \\
\hline 6 & C3 & GluE & & & \\
\hline 7 & C3 & GluE & & & \\
\hline 8 & C4 & GluE_cnid & none & LD 8-hr ; DD 24-hr & tentacles, column \\
\hline 9 & C4 & GluE_cnid & & & \\
\hline 10 & C4 & GluE_cnid & & & tentacles, column \\
\hline 11 & C2 & GluN_cnid & & & \\
\hline 12 & C4 & GluE_cnid & & & tentacles, column \\
\hline 13 & C2 & GluN_cnid & & & \\
\hline 14 & C2 & GluN_cnid & & & \\
\hline 15 & $\mathrm{C} 4$ & GluE_cnid & & & tentacles \\
\hline 16 & C2 & GluN_cnid & & & \\
\hline 17 & C2 & GluN_cnid & & & \\
\hline 18 & C2 & GluN_cnid & & & \\
\hline 19 & C2 & GluN_cnid & & & \\
\hline 20 & C3 & GluE & & & \\
\hline 21 & C2 & GluN_cnid & bacteria treatments & & tentacles \\
\hline 22 & C1 & GluN1 & & & tentacles, column \\
\hline 23 & C3 & GluE & bacteria treatments & & \\
\hline
\end{tabular}


Table 5.2 Summary of questions and hypotheses proposed in the disseration and concluding statements.

\begin{tabular}{|c|c|c|c|}
\hline QUESTION & PREDICTIONS & FINDING & CONCLUSION \\
\hline $\begin{array}{l}\text { 1a. What is the } \\
\text { extent of cnidarian } \\
\text { iGluR diversity? } \\
\text { Within the genome } \\
\text { of the sea } \\
\text { anemone, } \\
\text { Exaiptasia pallida? }\end{array}$ & N/A & N/A & $\begin{array}{l}\text { There are } 22 \text { Exaiptasia pallida iGluR } \\
\text { genes, one has two isoforms. These } \\
\text { genes are polyphyletic, occuring across } \\
\text { the evolution of iGluRs. }\end{array}$ \\
\hline $\begin{array}{l}\text { 1b. Are the protein } \\
\text { structures of } E \text {. } \\
\text { pallida iGluRs } \\
\text { reflective of } \\
\text { functional } \\
\text { characteristics } \\
\text { through conserved } \\
\text { domains? }\end{array}$ & N/A. & Supported & $\begin{array}{l}\text { Most GluRs have all or partially all } \\
\text { distinctly iGluR conserved domains. } \\
\text { Models of GluR with conserved domains } \\
\text { mirror the functional structure of iGluRs. }\end{array}$ \\
\hline $\begin{array}{l}\text { 1c. What level are } \\
\text { iGluRs involved } \\
\text { during the } \\
\text { response to } \\
\text { microbial } \\
\text { challenges? }\end{array}$ & $\begin{array}{l}\text { If cnidarian gluRs respond } \\
\text { to immune challenges } \\
\text { presented by bacteria, } \\
\text { expression will alter in } \\
\text { response to either } \\
\text { overwhelming signals or } \\
\text { to set physiological } \\
\text { responses in motion. }\end{array}$ & $\begin{array}{l}\text { Supported/ } \\
\text { Rejected }\end{array}$ & $\begin{array}{l}\text { Of the six tested E. pallida gluR genes, } \\
\text { four have significant transcriptional } \\
\text { responses in gene expression to bacterial } \\
\text { treatments. }\end{array}$ \\
\hline $\begin{array}{l}\text { 2a. Is there a daily } \\
\text { expression cycle for } \\
\text { cnidarian iGluRs? }\end{array}$ & $\begin{array}{l}\text { a. If cnidarian gluRs follow } \\
\text { physiological changes of } \\
\text { glutamate amounts, then } \\
\text { RNA transcription will } \\
\text { decrease following } \\
\text { glutamate synthesis. }\end{array}$ & Rejected & $\begin{array}{l}\text { gluR.2 and gluR8 expression cycles do } \\
\text { not follow changes in predicted } \\
\text { glutamate levels. Rather, the expression } \\
\text { changes with light cycle variation, i.e. } \\
\text { light absence. }\end{array}$ \\
\hline $\begin{array}{l}\text { 2b. Do cnidarian } \\
\text { iGluRs have a role } \\
\text { in biological } \\
\text { cycles? }\end{array}$ & $\begin{array}{l}\text { b. If cnidarian gluRs follow } \\
\text { a true circadian rhythm, } \\
\text { there will be one peak } \\
\text { and one trough within } 24 \\
\text { hours of sampling. }\end{array}$ & Rejected & $\begin{array}{l}\text { gluR8 had a } 24-\mathrm{hr} \text { period when in } \\
\text { constant darkness, but this did not hold } \\
\text { true in the } 12 \mathrm{hr} \text { light : } 12 \mathrm{hr} \text { dark } \\
\text { treatment. }\end{array}$ \\
\hline $\begin{array}{l}\text { 3. Where are iGluRs } \\
\text { localized within } E \text {. } \\
\text { pallida? }\end{array}$ & $\begin{array}{l}\text { Localization of gluRs is } \\
\text { likely to be within sensory } \\
\text { tentacle cells and } \\
\text { gastrodermal tissues that } \\
\text { phagocytize pathogenic } \\
\text { agents and within larvae } \\
\text { expressed in the apical } \\
\text { tuft. }\end{array}$ & $\begin{array}{l}\text { Supported/ } \\
\text { Rejected }\end{array}$ & $\begin{array}{l}\text { gluRs are localized in epidermal tissues, } \\
\text { particularly within tentacles around } \\
\text { sensory cells. Putatively involved in neuro- } \\
\text { nematocyst/spirocyst synapses. }\end{array}$ \\
\hline
\end{tabular}




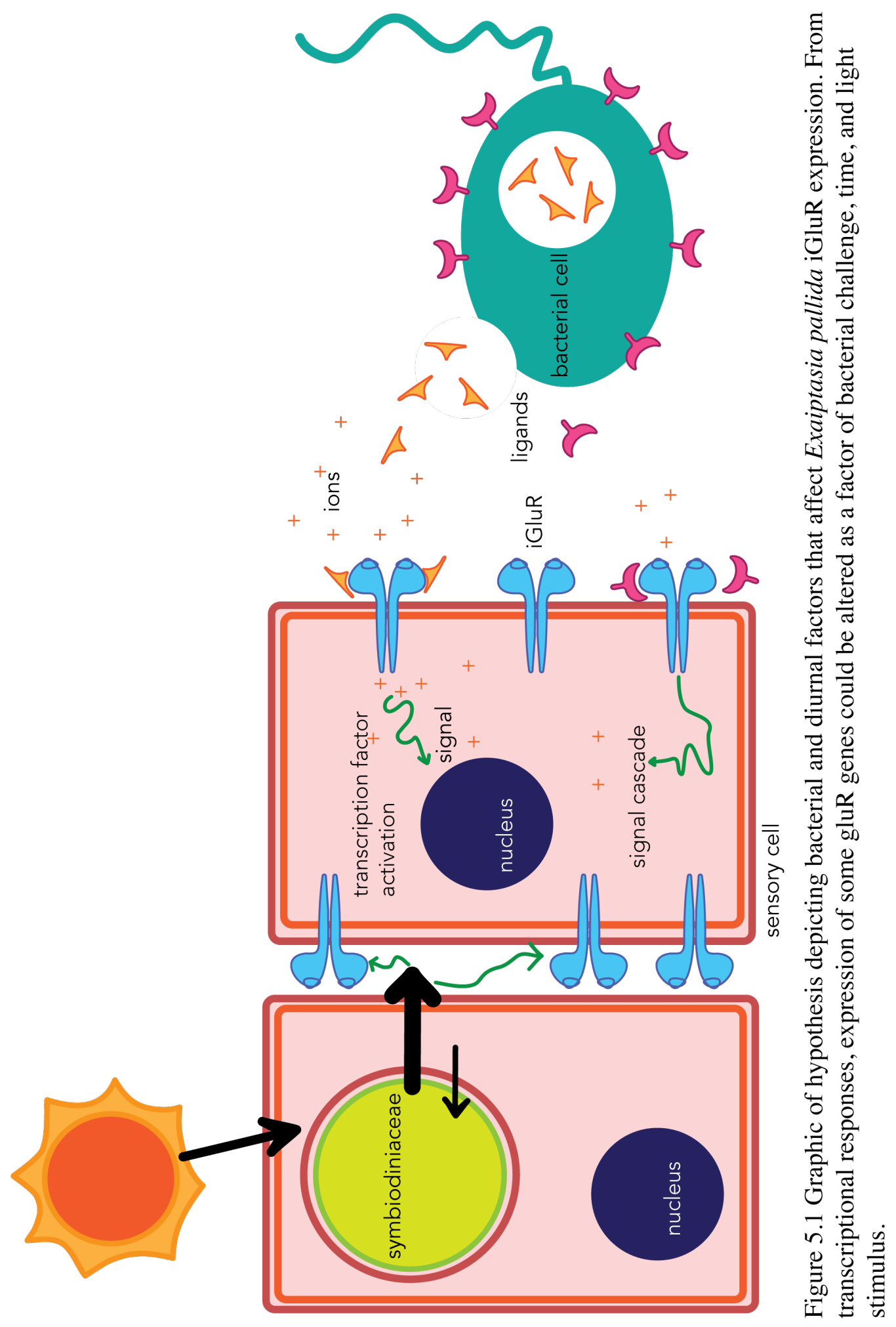




\subsection{References}

1. Sorek M, Schnytzer Y, Waldman Ben-Asher H, Chalifa Caspi V, Chen C-S, Miller DJ, et al. Setting the pace: host rhythmic behaviour and gene expression patterns in the facultatively symbiotic cnidarian Aiptasia are determined largely by Symbiodinium. Microbiome. 2018;6(83).

2. Pierobon P. Coordinated modulation of cellular signaling through ligand-gated ion channels in Hydra vulgaris (Cnidaria, Hydrozoa). Int J Dev Biol. 2012;56(68):551-65.

3. Westfall JA. Neural pathways and innervation of cnidocytes in tentacles of sea anemones. Hydrobiologia. 2004;530/531:117-21.

4. Stawski P, Janovjak H, Trauner D. Pharmacology of ionotropic glutamate receptors: A structural perspective. Bioorg Med Chem. 2010;18(22):7759-72.

5. Wudick MM, Michard E, Oliveira Nunes C, Feijó JA, Nunes CO, Feijó JA. Comparing plant and animal glutamate receptors: common traits but different fates? J Exp Bot. 2018;

6. Ramos-Vicente D, Ji J, Gratacòs-Batlle E, Gou G, Reig-Viader R, Luís J, et al. Metazoan evolution of glutamate receptors reveals unreported phylogenetic groups and divergent lineage-specific events. Elife. 2018;7:e35774.

7. Alberstein R, Grey R, Zimmet A, Simmons DK, Mayer ML. Glycine activated ion channel subunits encoded by ctenophore glutamate receptor genes. Proc Natl Acad Sci. 2015;10:E6048-57.

8. Cristofori-Armstrong B, Soh MS, Talwar S, Brown DL, Rash LD. Xenopus borealis as an alternative source of oocytes for biophysical and pharmacological studies of neuronal ion channels. Sci Rep. 2015;14763(October):1-12.

9. Dascal N. The Use of Xenopus Oocytes for the Study of Ion Channel. Crit Rev Biochem. 1987;22(4):317-87.

10. Chen PE, Wyllie DJA. Pharmacological insights obtained from structure function studies of ionotropic glutamate receptors. Br J Pharmacol. 2006;147:839_ 53.

11. Sorek M, Díaz-Almeyda EM, Medina M, Levy O. Circadian clocks in symbiotic corals: The duet between Symbiodinium algae and their coral host. Mar Genomics. 2014;14:47-57. 
12. Sorek M, Levy O. Influence of the Quantity and Quality of Light on Photosynthetic Periodicity in Coral Endosymbiotic Algae. PLoS One. 2012;7(8):e43264.

13. Shinzato C, Mungpakdee S, Satoh N, Shoguchi E. A genomic approach to coraldinoflagellate symbiosis: Studies of Acropora digitifera and Symbiodinium minutum. Front Microbiol. 2014;5(JULY):1-17.

14. Hughes ME, Abruzzi KC, Allada R, Anafi R, Arpat AB, Asher G, et al. Guidelines for Genome-Scale Analysis of Biological Rhythms. J Biol Rhythms. 2017;32(5):380-93.

15. Westfall JA, Landers DD, McCallum JD. Ultrastructure of neuro-spirocyte synapses in the sea anemone Aiptasia pallida (Cnidaria, Anthozoa, Zoantharia). J Morphol. 1999 Aug 1;241(2):165-73.

16. Brown T, Rodriguez-Lanetty M. Defending against pathogens - immunological priming and its molecular basis in a sea anemone, cnidarian. Sci Rep. $2015 ; 5: 17425$.

17. Wolenski FS, Layden MJ, Martindale MQ, Gilmore TD, Finnerty JR. Characterizing the spatiotemporal expression of RNAs and proteins in the starlet sea anemone, Nematostella vectensis. Nat Protoc. 2013;8(5):900-15.

18. Anokhin B, Hemmrich-Stanisak G, Bosch T. Karyotyping and single-gene detection using fluorescence in situ hybridization on chromosomes of Hydra magnipapillata (Cnidaria: Hydrozoa). Comp Cytogenet. 2010 Dec 30;4(2):97-110.

19. Westfall JA. Ultrastructure of systems synapses in the first-evolved nervous systems. J Neurocytol. 1996;25:735-46. 
VITA

\title{
ELLEN GRACE DOW
}

\author{
Born Portland, Oregon
}

2010-2014

B.S., Biology

Oregon State University

Corvallis, Oregon

$2014-2019$

Doctoral Candidate

Florida International University

Miami, Florida

Graduate Teaching Assistant

Florida International University

Miami, Florida

\section{PUBLICATIONS AND PRESENTATIONS}

Dow EG, J Siltberg Liberles and M Rodriguez-Lanetty. Submitted June 32019.

Cnidarian-specific Expansion of Ionotropic Glutamate Receptors Promote

Neofunctionalization with Implications for Chemosensory Immunity. Proceedings of the Royal Society B.

Camarena-Ubiera I, D Flores, EG Dow, A Cruz, J Alberte and T Pitzer (2019)

Understanding the Role of Gender in Mentorship Relationships and Interactions:

A Pilot. Presented at the Eighth Annual Peer-Led Team Learning International

Society Conference, Indianapolis, Indiana.

Dow EG and M Lagunas, B Maderal and M Rodriguez-Lanetty (January 3-7, 2019)

Cnidarian Ionotropic Glutamate Receptors under the clout of circadian rhythm in the sea anemone Exaiptasia pallida. Presented at the meeting of the Society for Integrative and Comparative Biology, Tampa, Florida.

Dow EG and M Rodriguez-Lanetty (July 2018) The Evolution of Function in Ionotropic Glutamate Receptors Respective to Host Signaling. Presented at the $9^{\text {th }}$ Annual International Symbiosis Society Congress, Corvallis, Oregon.

Flores D, I Camarena-Ubiera, EG Dow, A Cruz, J Alberte and T Pitzer (May, 2018) Initial Reliability Analysis for a 360 Evaluation of Peer Leaders. Presented at the Seventh Annual Peer-Led Team Learning International Society Conference, Dallas, Texas. 
Camarena-Ubiera I, D Flores, EG Dow, A Cruz, J Alberte and T Pitzer (May, 2018) Measuring Peer Leader Performance Using a 360 Evaluation. Presented at the Seventh Annual Peer-Led Team Learning International Society Conference, Dallas, Texas.

Dow EG and M Rodriguez-Lanetty (February 3, 2018) Function of Cnidarian-Specific Glutamate Receptors in Immunity. Lightening Talks, Presented at the Department of Biological Sciences Biosymposium, Miami, Florida.

Dow EG and M Rodriguez-Lanetty (January 3-7, 2018) A Cnidarian-Specific Glutamate Receptor Lineage Functional Immune Response. Presented at the meeting of the Society for Integrative and Comparative Biology, San Francisco, California.

Dow EG and M Rodriguez-Lanetty (June 9-14, 2016) A Cnidarian-Specific Ionotropic Glutamate Receptor Lineage Functionally Involved In Immunity. Presented at the $13^{\text {th }}$ International Coral Reef Symposium, Honolulu, Hawaii.

Dow EG and M Rodriguez-Lanetty (March 3-4, 2016) The role of iGluRs in Cnidarian Immunity. Presented at the meeting of Biomedical and Comparative Immunology, Miami, Florida.

Dow EG and M Rodriguez-Lanetty. (January 3-7, 2016) A new ionotropic glutamate receptor lineage and its putative role in cnidarian immunity. Presented at the meeting of the Society for Integrative and Comparative Biology, Portland, Oregon.

Dow EG, AK Barner, AZ Poole, and VM Weis. (January 3-7, 2015) Effects of light and thermal variation on symbiotic and aposymbiotic states of the temperate sea anemone, Anthopleura elegantissima. Presented at the meeting of the Society for Integrative and Comparative Biology, West Palm Beach, Florida.

Dow EG. (May 22, 2014) "Rabón" from de Puerto Lopez, Presented at the Prism Contributor's Showcase, Corvallis, Oregon.

Dow EG. (June 7, 2013) Effects of light and temperature variations on the symbiosis of the temperature anemone, Anthopleura elegantissima. Presented at the OSU/Hatfield Marine Science Center Marine Biology Symposium, Newport, Oregon.

Dow EG, AZ Poole and VM Weis (September 15-17, 2012) The Role of Complement Molecules in Cnidarians, Presented at the OSU/HHMI Summer Undergraduate Research Program Symposium, Corvallis, Oregon. 